\title{
Synthesis of Glycosyl Boranophosphates and Their Applications as Precursors of Glycosyl Phosphate Analogs
}

Fumiko Matsumura, Natsuhisa Oka, and Takeshi Wada*

Department of Medical Genome Sciences, Graduate School of Frontier Sciences, The University of Tokyo, Bioscience Building 702, 5-1-5 Kashiwanoha, Kashiwa, Chiba 277-8562, Japan

wada@k.u-tokyo.ac.jp

\section{Supporting Information}

(A)

(B)

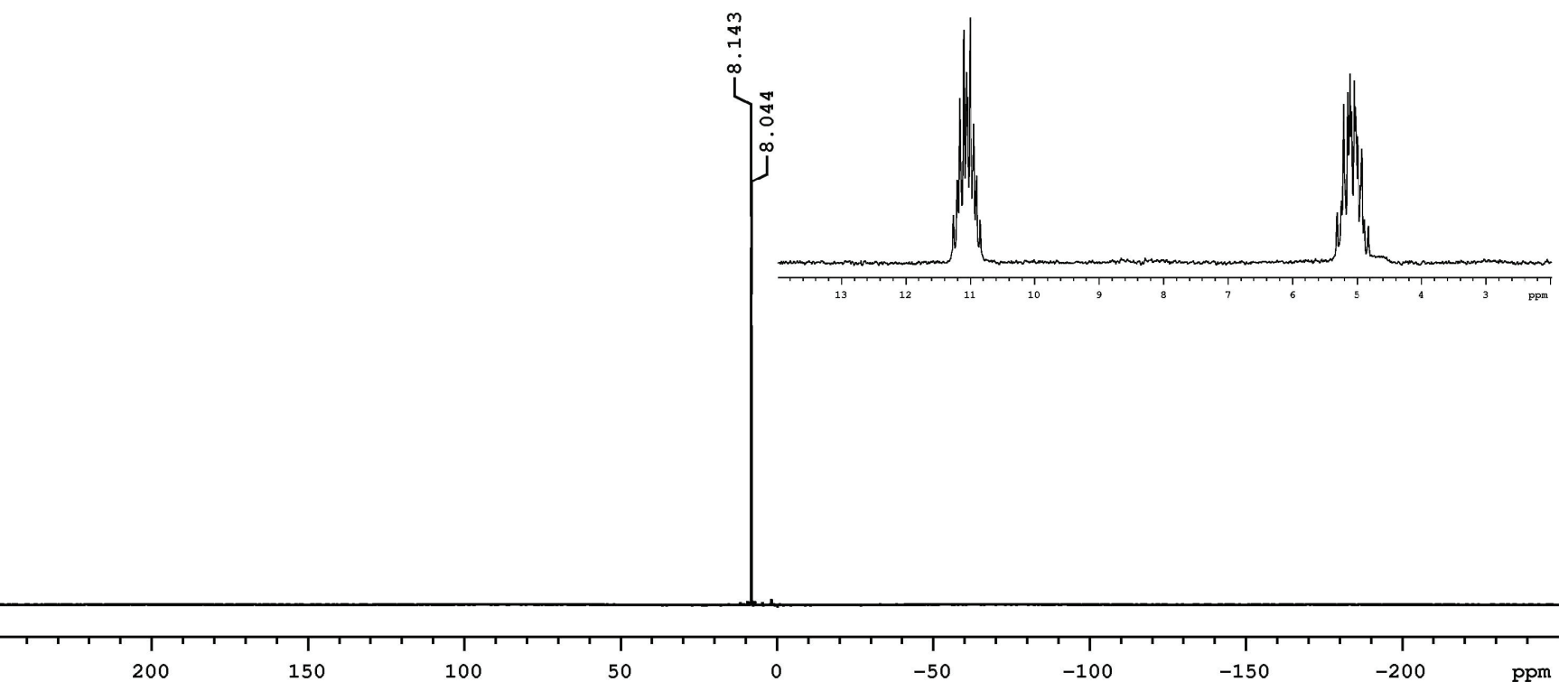

${ }^{31} \mathrm{P}$ NMR spectra of 19 with (A) P-H decoupling and (B) P-H coupling $\left(\mathrm{CDCl}_{3}, 121.5 \mathrm{MHz}\right)$.

\section{General}

IR spectra were recorded on a JASCO FT/IR-480 Plus spectrophotometer. All NMR spectra were recorded on a Varian Mercury 300. ${ }^{1} \mathrm{H}$ NMR spectra were obtained at $300 \mathrm{MHz}$ with tetramethylsilane (TMS) ( $\left.\delta 0.0\right)$ as an internal standard in $\mathrm{CDCl}_{3}$ and $\mathrm{CD}_{3} \mathrm{CN} .{ }^{13} \mathrm{C}$ NMR spectra were obtained at $75.5 \mathrm{MHz}$ with $\mathrm{CDCl}_{3}$ as an internal standard $(\delta$ 77.0) in $\mathrm{CDCl}_{3}$, or with $\mathrm{CD}_{3} \mathrm{CN}(\delta 118.0)$ as an internal standard in $\mathrm{CD}_{3} \mathrm{CN} .{ }^{31} \mathrm{P}$ NMR spectra were obtained at 121.5 $\mathrm{MHz}$ with $85 \% \mathrm{H}_{3} \mathrm{PO}_{4}(\delta 0.0)$ as an external standard. ESI mass spectra were recorded on an Applied Biosystems QSTAR. FAB mass spectra were recorded on a JEOL JMS-HX110 spectrometer. All the reactions except for the conversion of $\mathbf{2 3}$ to $\mathbf{2 4}$ were conducted under an inert atmosphere. Silica gel column chromatography was carried out using Kanto silica gel 60 N, 63-210 $\mu \mathrm{m}$. Analytical TLC was performed on Merck Kieselgel $60-\mathrm{F}_{254}$ plates. Dry organic solvents were prepared by appropriate procedures prior to use. The other organic solvents were reagent grade and used as received.

Dimethyl 2,3,4,6-tetra-O-benzyl-D-glucopyranosyl boranophosphate (10).

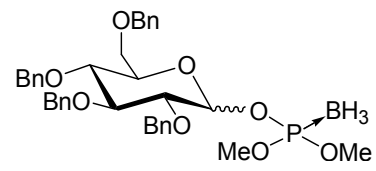


Reducing sugar $2(271 \mathrm{mg}, 0.50 \mathrm{mmol})$ and $i$ - $\operatorname{Pr}_{2} \mathrm{NEt}(0.85 \mathrm{~mL}, 5.0 \mathrm{mmol})$ were added to a $0.3 \mathrm{M}$ solution of triethylammonium dimethyl boranophosphate 1 in dry THF $(5.0 \mathrm{~mL})$, which was dried over MS 4A prior to use. NT (287 mg, $2.5 \mathrm{mmol})$ and Bop-Cl $(634 \mathrm{mg}, 2.5 \mathrm{mmol})$ were added successively to the solution, and the mixture was stirred for $1 \mathrm{~h}$ at $\mathrm{rt}$. The mixture was then diluted with saturated $\mathrm{NaHCO}_{3}$ aqueous solution $(5 \mathrm{~mL})$ and $\mathrm{CH}_{2} \mathrm{Cl}_{2}(30 \mathrm{~mL})$. The separated organic layer was washed four times with saturated $\mathrm{NaHCO}_{3}$ aqueous solution $(4 \times 30 \mathrm{~mL})$, dried over $\mathrm{Na}_{2} \mathrm{SO}_{4}$, filtered and concentrated under reduced pressure. The residue was purified by column chromatography [hexane-ethyl acetate $(4: 1, \mathrm{v} / \mathrm{v})$ ] on silica gel to afford $10(292 \mathrm{mg}, 0.45 \mathrm{mmol}, 90 \%, \alpha: \beta=4: 6)$. Colorless oil. IR (KBr, $\left.\mathrm{cm}^{-1}\right)$ 3088, 3063, 3031, 2953, 2907, 2867, 2400, 2356, 1496, 1454, 1361, 1209, 1178, 1150, 1093, 1028, 943, 867, 834, 737, 698. ${ }^{1} \mathrm{H} \mathrm{NMR}\left(\mathrm{CDCl}_{3}\right) \delta 7.40-7.10\left(\mathrm{~m}, \mathrm{CH}_{2} \underline{\mathrm{Ph}}\right), 5.80(\mathrm{dd}, J=3.3 \mathrm{~Hz}, 7.7 \mathrm{~Hz}, \alpha \mathrm{H}-1), 5.10(\mathrm{t}, J=7.4 \mathrm{~Hz}, \beta \mathrm{H}-1)$, 4.96-4.43 (m, $\mathrm{CH}_{2} \mathrm{Ph}$ ), 3.97-3.51 (m, $\left.\alpha \mathrm{H}-2-\alpha \mathrm{H}-6, \beta \mathrm{H}-2-\beta \mathrm{H}-6, \alpha \mathrm{POC}_{3}, \beta \mathrm{POC}_{3}\right), 1.2$ to $-0.2\left(\mathrm{br}, \mathrm{BH}_{3}\right) .{ }^{31} \mathrm{P} \mathrm{NMR}$ $\left(\mathrm{CDCl}_{3}\right) \delta 121.4-116.5(\mathrm{~m}) .{ }^{13} \mathrm{C}$ NMR $\left(\mathrm{CDCl}_{3}\right) \delta 97.9\left(\mathrm{~d}, J_{\mathrm{C}-\mathrm{P}}=3.7 \mathrm{~Hz}, \beta \mathrm{C}-1\right), 94.6\left(\mathrm{~d}, J_{\mathrm{C}-\mathrm{P}}=2.3 \mathrm{~Hz}, \alpha \mathrm{C}-1\right) . \mathrm{HRMS}$ (ESI): calcd for $[\mathrm{M}+\mathrm{H}]^{+}: 647.2947$; found: 647.2946 .

Dimethyl 2,3,4,6-tetra- $O$-acetyl-D-glucopyranosyl boranophosphate (11).

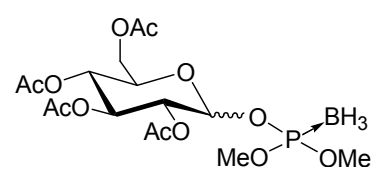

Reducing sugar $3(348 \mathrm{mg}, 1.0 \mathrm{mmol})$ and $i$ - $\mathrm{Pr}_{2} \mathrm{NEt}(1.7 \mathrm{~mL}, 10 \mathrm{mmol})$ were added to a $0.3 \mathrm{M}$ solution of 1 in dry THF (10 mL), which was dried over MS 4A prior to use. NT (558 mg, $4.9 \mathrm{mmol})$ and Bop-Cl (1.28 g, $5.0 \mathrm{mmol})$ were added successively to the solution, and the mixture was stirred for $1 \mathrm{~h}$ at $\mathrm{rt}$. The mixture was then diluted with saturated $\mathrm{NaHCO}_{3}$ aqueous solution $(10 \mathrm{~mL})$ and $\mathrm{CH}_{2} \mathrm{Cl}_{2}(50 \mathrm{~mL})$. The separated organic layer was washed four times with saturated $\mathrm{NaHCO}_{3}$ aqueous solution $(4 \times 50 \mathrm{~mL})$, dried over $\mathrm{Na}_{2} \mathrm{SO}_{4}$, filtered and concentrated under reduced pressure. The residue was purified by column chromatography [hexane-ethyl acetate $(2: 1, \mathrm{v} / \mathrm{v})$ ] on silica gel to afford 11 (400 mg, 0.88 mmol, 88\%, $\alpha: \beta=5: 5)$. Colorless solid. IR (KBr, $\left.\mathrm{cm}^{-1}\right)$ 2959, 2854, 2404, 2359, 1754, 1437, 1369, 1224, 1150 , 1036, 949, 839. ${ }^{1} \mathrm{H}$ NMR $\left(\mathrm{CDCl}_{3}\right) \delta 5.81(\mathrm{dd}, J=3.4 \mathrm{~Hz}, 7.9 \mathrm{~Hz}, \alpha \mathrm{H}-1), 5.48(\mathrm{t}, J=9.8 \mathrm{~Hz}, \alpha \mathrm{H}-3), 5.28-4.97$ (m, $\alpha \mathrm{H}-2$, . $\alpha \mathrm{H}-4, \beta \mathrm{H}-1-\beta \mathrm{H}-4), 4.29-4.08$ (m, $\alpha \mathrm{H}-5, \alpha \mathrm{H}-6, \beta \mathrm{H}-6), 3.78-3.68\left(\mathrm{~m}, \beta \mathrm{H}-5, \alpha \mathrm{POCH}_{3}, \beta \mathrm{POC} \underline{H}_{3}\right), 2.08-2.00$ (m, $\left.\mathrm{COCH}_{3}\right), 1.2$ to $-0.2\left(\mathrm{br}, \underline{\mathrm{BH}}_{3}\right) .{ }^{31} \mathrm{P} \mathrm{NMR}\left(\mathrm{CDCl}_{3}\right) \delta 121.2-117.0(\mathrm{~m}) .{ }^{13} \mathrm{C} \mathrm{NMR}\left(\mathrm{CDCl}_{3}\right) \delta 95.7(\beta \mathrm{C}-1), 92.8\left(\mathrm{~d}, J_{\mathrm{C}-\mathrm{P}}=\right.$ $1.7 \mathrm{~Hz}, \alpha \mathrm{C}-1)$. HRMS (ESI): calcd for $[\mathrm{M}-\mathrm{H}]^{+}: 453.1331$; found: 453.1329.

Dimethyl 2,3,4,6-tetra- $O$-acetyl- $\beta$-D-glucopyranosyl boranophosphate (11 $\beta$ ).

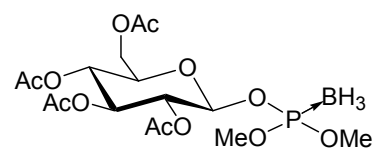

The $\alpha \beta$ mixture of 11 (4.73 g, $10 \mathrm{mmol})$ was purified by column chromatography [hexane-ethyl acetate $(2: 1, \mathrm{v} / \mathrm{v})$ ] on silica gel to afford $\mathbf{1 1} \beta(1.08 \mathrm{~g}, 2.4 \mathrm{mmol}, 23 \%)$. IR (KBr, $\left.\mathrm{cm}^{-1}\right)$ 2960, 2854, 2404, 2359, 1754, 1440, 1368, 1222, 1032 , 839. ${ }^{1} \mathrm{H}$ NMR $\left(\mathrm{CDCl}_{3}\right) \delta$ 5.27-5.16 (m, 2H, H-1, H-3), 5.11-5.05 (m, 2H, H-2, H-4), 4.26 (dd, J=5.2 Hz, $12.4 \mathrm{~Hz}, 1 \mathrm{H}$,

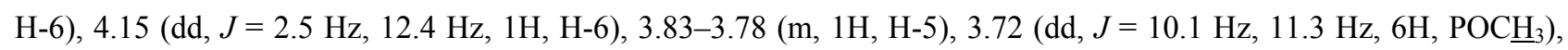
$2.09\left(\mathrm{~s}, 3 \mathrm{H}, \mathrm{COCH}_{3}\right), 2.08\left(\mathrm{~s}, 3 \mathrm{H}, \mathrm{COC}_{3}\right), 2.04\left(\mathrm{~s}, 3 \mathrm{H}, \mathrm{COCH}_{3}\right), 2.02\left(\mathrm{~s}, 3 \mathrm{H}, \mathrm{COCH}_{3}\right), 1.2$ to $-0.1\left(\mathrm{br}, 1 \mathrm{H}, \mathrm{BH}_{3}\right) .{ }^{31} \mathrm{P}$ $\operatorname{NMR}\left(\mathrm{CDCl}_{3}\right) \delta$ 121.4-117 4(m). ${ }^{13} \mathrm{C}$ NMR $\left(\mathrm{CDCl}_{3}\right) \delta 170.3,169.8,169.3,169.1\left(\mathrm{COCH}_{3}\right), 95.7$ (C-1), $72.7(\mathrm{C}-5)$, $72.2(\mathrm{C}-3), 71.2$ (d, $\left.J_{\mathrm{C}-\mathrm{P}}=6.9 \mathrm{~Hz}, \mathrm{C}-2\right), 67.9$ (C-4), 61.5 (C-6), 53.5 (dd $\left.J=2.0,5.2 \mathrm{~Hz}, \mathrm{POCH}_{3}\right), 20.8,20.7\left(\mathrm{COCH}_{3}\right)$. HRMS (ESI): calcd for [M-H] $]^{+}$453.1331; found: 453.1329. 


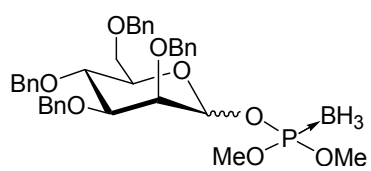

Reducing sugar 4 (163 mg, $0.30 \mathrm{mmol}), 1$ (202 $\mathrm{mg}, 0.90 \mathrm{mmol})$, which was dried by repeated coevaporations with dry toluene, and $i$-Pr ${ }_{2} \mathrm{NEt}(0.51 \mathrm{~mL}, 3.0 \mathrm{mmol})$ were dissolved in dry THF $(3.0 \mathrm{~mL})$. NT $(171 \mathrm{mg}, 1.5 \mathrm{mmol})$ and Bop-Cl (382 mg, $1.5 \mathrm{mmol})$ were added successively to the solution, and the mixture was stirred for $1 \mathrm{~h}$ at $\mathrm{rt}$. The mixture was then diluted with saturated $\mathrm{NaHCO}_{3}$ aqueous solution $(3 \mathrm{~mL})$ and $\mathrm{CH}_{2} \mathrm{Cl}_{2}(30 \mathrm{~mL})$. The separated organic layer was washed four times with saturated $\mathrm{NaHCO}_{3}$ aqueous solution $(4 \times 30 \mathrm{~mL})$, dried over $\mathrm{Na}_{2} \mathrm{SO}_{4}$, filtered and concentrated under reduced pressure. The residue was purified by column chromatography [hexane-ethyl acetate (4:1, $\mathrm{v} / \mathrm{v})$ ] on silica gel to afford 12 (165 mg, $0.25 \mathrm{mmol}, 85 \%, \alpha: \beta=8: 2)$. Colorless oil. IR $\left(\mathrm{KBr}, \mathrm{cm}^{-1}\right) 3059,3022,2947$, 2910, 2866, 2397, 2359, 1499, 1454, 1362, 1173, 1099, 1029, 937, 835, 738, 698. ${ }^{1} \mathrm{H}$ NMR $\left(\mathrm{CDCl}_{3}\right) \delta$ 7.42-7.15 (m, $\mathrm{CH}_{2} \mathrm{Ph}$ ), 5.69 (dd, $\left.J=1.9 \mathrm{~Hz}, 7.7 \mathrm{~Hz}, \alpha \mathrm{H}-1\right), 5.14(\mathrm{~d}, J=7.2 \mathrm{~Hz}, \beta \mathrm{H}-1), 4.90-4.47\left(\mathrm{~m}, \mathrm{C}_{2} \mathrm{Ph}\right), 4.06-3.52(\mathrm{~m}$, $\left.\alpha \mathrm{H}-2-\alpha \mathrm{H}-6, \beta \mathrm{H}-2-\beta \mathrm{H}-6, \alpha \mathrm{POCH}_{3}, \beta \mathrm{POCH}_{3}\right), 1.2$ to $-0.2\left(\mathrm{br}, \mathrm{BH}_{3}\right) .{ }^{31} \mathrm{P} \mathrm{NMR}\left(\mathrm{CDCl}_{3}\right) \delta 121.4-116.5$ (m). ${ }^{13} \mathrm{C} \mathrm{NMR}$ $\left(\mathrm{CDCl}_{3}\right) \delta 96.3(\beta \mathrm{C}-1), 95.1$ (d, $\left.J_{\mathrm{C}-\mathrm{P}}=3.2 \mathrm{~Hz}, \alpha \mathrm{C}-1\right)$. HRMS (ESI): calcd for [M+H] $]^{+}$: 647.2947; found: 647.2949.

\section{Dimethyl 2,3,4,6-tetra- $O$-acetyl-D-mannopyranosyl boranophosphate (13).}

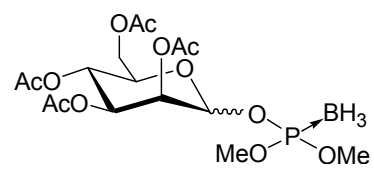

Reducing sugar $5(393 \mathrm{mg}, 1.1 \mathrm{mmol})$ and $i-\operatorname{Pr}_{2} \mathrm{NEt}(1.9 \mathrm{~mL}, 11 \mathrm{mmol})$ were added to a $0.3 \mathrm{M}$ solution of 1 in dry THF (11 mL), which was dried over MS 4A prior to use. NT (644 mg, $5.7 \mathrm{mmol})$ and Bop-Cl (1.43 g, $5.7 \mathrm{mmol})$ were added successively to the solution, and the mixture was stirred for $1 \mathrm{~h}$ at $\mathrm{rt}$. The mixture was then diluted with saturated $\mathrm{NaHCO}_{3}$ aqueous solution $(10 \mathrm{~mL})$ and $\mathrm{CH}_{2} \mathrm{Cl}_{2}(50 \mathrm{~mL})$. The separated organic layer was washed four times with saturated $\mathrm{NaHCO}_{3}$ aqueous solution $(4 \times 50 \mathrm{~mL})$, dried over $\mathrm{Na}_{2} \mathrm{SO}_{4}$, filtered and concentrated under reduced pressure. The residue was purified by column chromatography [hexane-ethyl acetate $(2: 1, \mathrm{v} / \mathrm{v})$ ] on silica gel to afford $\mathbf{1 3}$ (399 $\mathrm{mg}$, $0.88 \mathrm{mmol}, 78 \%, \alpha: \beta=7: 3)$. Colorless oil. IR $\left(\mathrm{KBr}, \mathrm{cm}^{-1}\right) 2956,2854,2405,2359,1752,1437,1370,1223,1164,1033$, 962, 838. ${ }^{1} \mathrm{H}$ NMR $\left(\mathrm{CDCl}_{3}\right) \delta 5.61(\mathrm{dd}, J=1.7 \mathrm{~Hz}, 7.4 \mathrm{~Hz}, \alpha \mathrm{H}-1), 5.49(\mathrm{~d}, J=3.0 \mathrm{~Hz}, \beta \mathrm{H}-2), 5.40-5.11$ (m, $\alpha \mathrm{H}-2-\alpha \mathrm{H}-4$ and $\beta \mathrm{H}-1, \beta \mathrm{H}-3, \beta \mathrm{H}-4), 4.33-4.09$ (m, $\alpha \mathrm{H}-5, \alpha \mathrm{H}-6, \beta \mathrm{H}-5, \beta \mathrm{H}-6$ ), 3.79 (dd, $\alpha \mathrm{POC} \underline{H}_{3}, J=5.0 \mathrm{~Hz}, 11.3 \mathrm{~Hz}$ ), $3.72\left(\mathrm{dd}, \beta \mathrm{POC}_{3}, J=9.0 \mathrm{~Hz}, 11.3 \mathrm{~Hz}\right), 2.20-2.01\left(\mathrm{~m}, \mathrm{COCH}_{3}\right), 1.2$ to $-0.2\left(\mathrm{br}, \mathrm{BH}_{3}\right) .{ }^{31} \mathrm{P}$ NMR $\left(\mathrm{CDCl}_{3}\right) \delta$ 121.2-117.0 (m). ${ }^{13} \mathrm{C}$ NMR $\left(\mathrm{CDCl}_{3}\right) \delta 93.9\left(\mathrm{~d}, J_{\mathrm{C}-\mathrm{P}}=2.6 \mathrm{~Hz}, \alpha \mathrm{C}-1\right), 93.4(\beta \mathrm{C}-1)$. HRMS (ESI): calcd for [M-H] ${ }^{+}$: 453.1331; found: 453.1331 .

Dimethyl 2,3,4,6-tetra- $O$-benzyl-D-galactopyranosyl boranophosphate (14).

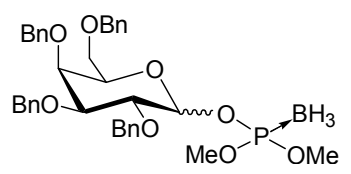

Reducing sugar 6 (271 mg, $0.50 \mathrm{mmol}), \mathbf{1}(337 \mathrm{mg}, 1.5 \mathrm{mmol})$, which was dried by repeated coevaporations with dry toluene, and $i$ - $\mathrm{Pr}_{2} \mathrm{NEt}(0.85 \mathrm{~mL}, 5.0 \mathrm{mmol})$ were dissolved in dry THF $(5.0 \mathrm{~mL})$. NT $(288 \mathrm{mg}, 2.5 \mathrm{mmol})$ and Bop-Cl (638 mg, $2.5 \mathrm{mmol}$ ) were added successively to the solution, and the mixture was stirred for $1 \mathrm{~h}$ at $\mathrm{rt}$. The mixture was then diluted with saturated $\mathrm{NaHCO}_{3}$ aqueous solution $(5 \mathrm{~mL})$ and $\mathrm{CH}_{2} \mathrm{Cl}_{2}(30 \mathrm{~mL})$. The separated organic layer was washed four times with saturated $\mathrm{NaHCO}_{3}$ aqueous solution $(4 \times 30 \mathrm{~mL})$, dried over $\mathrm{Na}_{2} \mathrm{SO}_{4}$, filtered and concentrated 
under reduced pressure. The residue was purified by column chromatography [hexane-ethyl acetate $(4: 1, \mathrm{v} / \mathrm{v})$ ] on silica gel to afford 14 (284 mg, $0.44 \mathrm{mmol}, 88 \%, \alpha: \beta=8: 2)$. Colorless oil. IR $\left(\mathrm{KBr}, \mathrm{cm}^{-1}\right)$ 3059, 3030, 2947, 2869, 2399, 2359, 1496, 1454, 1363, 1102, 1028, 936, 834, 737, 697. ${ }^{1} \mathrm{H}$ NMR $\left(\mathrm{CDCl}_{3}\right) \delta$ 7.40-7.20 (m, $\left.\mathrm{CH}_{2} \underline{\mathrm{Ph}}\right), 5.81(\mathrm{dd}, J=3.5$ $\mathrm{Hz}, 7.6 \mathrm{~Hz}, \alpha \mathrm{H}-1), 5.06$ (t, $J=7.7 \mathrm{~Hz}, \beta \mathrm{H}-1), 4.95-4.36$ (m, $\underline{\mathrm{H}}_{2} \mathrm{Ph}$ ), 4.12-3.86 (m, $\alpha \mathrm{H}-2, \alpha \mathrm{H}-3, \alpha \mathrm{H}-4, \alpha \mathrm{H}-5, \beta \mathrm{H}-2$, $\beta \mathrm{H}-4$ ), 3.71-3.54 (m, $\left.\alpha \mathrm{H}-6, \beta \mathrm{H}-3, \beta \mathrm{H}-5, \beta \mathrm{H}-6, \alpha \mathrm{POC} \underline{H}_{3}, \beta \mathrm{POC} \underline{H}_{3}\right), 1.2$ to -0.1 (br, $\left.\underline{B}_{3}\right) .{ }^{31} \mathrm{P} \mathrm{NMR}\left(\mathrm{CDCl}_{3}\right) \delta$ 121.4-116.8 (m). ${ }^{13} \mathrm{C}$ NMR $\left(\mathrm{CDCl}_{3}\right) \delta 98.2\left(\mathrm{~d}, J_{\mathrm{C}-\mathrm{P}}=3.5 \mathrm{~Hz}, \beta \mathrm{C}-1\right), 95.4$ (d, $\left.J_{\mathrm{C}-\mathrm{P}}=2.9 \mathrm{~Hz}, \alpha \mathrm{C}-1\right)$. HRMS (ESI): calcd for $[\mathrm{M}+\mathrm{H}]^{+}: 647.2947$; found: 647.2944 .

\section{Dimethyl 2,3,4,6-tetra- $O$-acetyl-D-galactopyranosyl boranophosphate (15).}

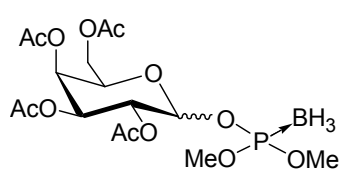

Reducing sugar 7 (175 mg, $0.50 \mathrm{mmol}), \mathbf{1}(338 \mathrm{mg}, 1.5 \mathrm{mmol})$, which was dried by repeated coevaporations with dry toluene, and $i$ - $\operatorname{Pr}_{2} \mathrm{NEt}(0.85 \mathrm{~mL}, 5.0 \mathrm{mmol})$ were dissolved in dry THF $(5.0 \mathrm{~mL})$. NT (288 mg, $\left.2.5 \mathrm{mmol}\right)$ and Bop-Cl (639 $\mathrm{mg}, 2.5 \mathrm{mmol}$ ) were added successively to the solution, and the mixture was stirred for $1 \mathrm{~h}$ at $\mathrm{rt}$. The mixture was then diluted with saturated $\mathrm{NaHCO}_{3}$ aqueous solution $(5 \mathrm{~mL})$ and $\mathrm{CH}_{2} \mathrm{Cl}_{2}(30 \mathrm{~mL})$. The separated organic layer was washed four times with saturated $\mathrm{NaHCO}_{3}$ aqueous solution $(4 \times 30 \mathrm{~mL})$, dried over $\mathrm{Na}_{2} \mathrm{SO}_{4}$, filtered and concentrated under reduced pressure. The residue was purified by column chromatography [hexane-ethyl acetate $(2: 1, \mathrm{v} / \mathrm{v})]$ on silica gel to afford 15 (208 mg, $0.46 \mathrm{mmol}, 91 \%, \alpha: \beta=5: 5)$. Colorless oil. IR $\left(\mathrm{KBr}, \mathrm{cm}^{-1}\right)$ 2959, 2854, 2404, 2359, 1751, 1438, 1371, 1221, 1071, 1031, 945, 839. ' H NMR $\left(\mathrm{CDCl}_{3}\right) \delta 5.86(\mathrm{dd}, J=3.3 \mathrm{~Hz}, 7.7 \mathrm{~Hz}, \alpha \mathrm{H}-1), 5.49(\mathrm{dd}, J=1.2 \mathrm{~Hz}$, $3.2 \mathrm{~Hz}, \alpha \mathrm{H}-4), 5.41$ (dd, $J=1.1 \mathrm{~Hz}, 3.3 \mathrm{~Hz}, \beta \mathrm{H}-4), 5.37-5.20$ (m, $\alpha \mathrm{H}-2, \alpha \mathrm{H}-3, \beta \mathrm{H}-2), 5.16(\mathrm{t}, J=7.7 \mathrm{~Hz}, \beta \mathrm{H}-1), 5.07$ (dd, $J=3.3 \mathrm{~Hz}, 10.5 \mathrm{~Hz}, \beta \mathrm{H}-3), 4.42-4.00$ (m, $\alpha \mathrm{H}-5, \alpha \mathrm{H}-6, \beta \mathrm{H}-5, \beta \mathrm{H}-6), 3.80-3.70\left(\mathrm{~m}, \alpha \mathrm{POC}_{3}, \beta \mathrm{POCH}_{3}\right.$ ), 2.17-2.00 (m, $\left.\mathrm{COC} \underline{H}_{3}\right), 1.2$ to $-0.2\left(\mathrm{br}, \underline{\mathrm{BH}}_{3}\right) .{ }^{31} \mathrm{P} \mathrm{NMR}\left(\mathrm{CDCl}_{3}\right) \delta 121.2-117.0(\mathrm{~m}) .{ }^{13} \mathrm{C} \mathrm{NMR}\left(\mathrm{CDCl}_{3}\right) \delta 96.1(\beta \mathrm{C}-1)$, $93.5\left(\mathrm{~d}, J_{\mathrm{C}-\mathrm{P}}=2.0 \mathrm{~Hz}, \alpha \mathrm{C}-1\right)$. HRMS (ESI): calcd for [M-H] $]^{+}$: 453.1331; found: 453.1332.

Dimethyl 2-acetamide-3,4,6-tri- $O$-acetyl-2-deoxy-D-glucopyranosyl boranophosphate (16).

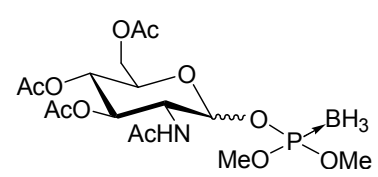

Reducing sugar $8(101 \mathrm{mg}, 0.29 \mathrm{mmol})$ and $i$-Pr $2 \mathrm{NEt}(0.51 \mathrm{~mL}, 3.0 \mathrm{mmol})$ were added to a $0.3 \mathrm{M}$ solution of triethylammonium dimethyl boranophosphate $\mathbf{1}$ in dry THF $(3.0 \mathrm{~mL})$, which was dried over MS 4A prior to use. NT (171 mg, $1.5 \mathrm{mmol})$ and Bop-Cl (383 mg, $1.5 \mathrm{mmol})$ were added successively to the solution, and the mixture was stirred for $1 \mathrm{~h}$ at $\mathrm{rt}$. The mixture was then diluted with saturated $\mathrm{NaHCO}_{3}$ aqueous solution $(3 \mathrm{~mL})$ and $\mathrm{CH}_{2} \mathrm{Cl}_{2}(20 \mathrm{~mL})$. The separated organic layer was washed four times with saturated $\mathrm{NaHCO}_{3}$ aqueous solution $(4 \times 20 \mathrm{~mL})$, dried over $\mathrm{Na}_{2} \mathrm{SO}_{4}$, filtered and concentrated under reduced pressure. The residue was purified by column chromatography (ethyl acetate) on silica gel to afford 16 ( $73.5 \mathrm{mg}, 0.16 \mathrm{mmol}, 56 \%, \alpha: \beta=8: 2)$. Colorless solid. IR $\left(\mathrm{KBr}, \mathrm{cm}^{-1}\right) 3627,3285$, 2958, 2854, 2402, 2369, 1749, 1664, 1543, 1442, 1369, 1232, 1134, 1035, 939, 835. ${ }^{1} \mathrm{H}$ NMR $\left(\mathrm{CDCl}_{3}\right) \delta 5.71-5.58$ ( $\alpha \mathrm{NH}, \beta \mathrm{NH}, \alpha \mathrm{H}-1), 5.27-5.14$ (m, $\alpha \mathrm{H}-3, \alpha \mathrm{H}-4, \beta \mathrm{H}-1, \beta \mathrm{H}-3, \beta \mathrm{H}-4), 4.39-4.35$ (m, $\alpha \mathrm{H}-2), 4.30-4.10$ (m, $\alpha \mathrm{H}-5$, $\alpha \mathrm{H}-6, \beta \mathrm{H}-2), 3.83-3.67$ (m, $\left.\alpha \mathrm{POC} \underline{H}_{3}, \beta \mathrm{POC} \underline{H}_{3}\right),-0.1$ to -1.2 (br, $\left.\mathrm{B}_{3}\right) .{ }^{31} \mathrm{P}$ NMR $\left(\mathrm{CDCl}_{3}\right) \delta 122.0-116.0(\mathrm{~m}) .{ }^{13} \mathrm{C}$ $\operatorname{NMR}\left(\mathrm{CDCl}_{3}\right) \delta 95.6(\beta \mathrm{C}-1), 92.5\left(\mathrm{~d}, J_{\mathrm{C}-\mathrm{P}}=4.9 \mathrm{~Hz}, \alpha \mathrm{C}-1\right)$. HRMS (ESI): calcd for $[\mathrm{M}-\mathrm{H}]^{+}: 452.1490$; found: 452.1489 . 
Methyl 1-O-dimethoxyboranophosphinyl-2,3,4-tri-O-benzyl-D-glucopyranuronate (17).

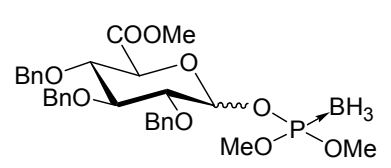

Reducing sugar 9 (71.6 mg, $0.15 \mathrm{mmol}), 1(101 \mathrm{mg}, 0.45 \mathrm{mmol})$, which was dried by repeated coevaporations with dry toluene, and $i-\mathrm{Pr}_{2} \mathrm{NEt}(0.25 \mathrm{~mL}, 1.5 \mathrm{mmol})$ were dissolved in dry THF $(5.0 \mathrm{~mL})$. NT $(85.5 \mathrm{mg}, 0.75 \mathrm{mmol})$ and Bop-Cl $(176 \mathrm{mg}, 0.70 \mathrm{mmol})$ were added successively to the solution, and the mixture was stirred for $1 \mathrm{~h}$ at $\mathrm{rt}$. The mixture was then diluted with saturated $\mathrm{NaHCO}_{3}$ aqueous solution $(2 \mathrm{~mL})$ and $\mathrm{CH}_{2} \mathrm{Cl}_{2}(15 \mathrm{~mL})$. The separated organic layer was washed four times with saturated $\mathrm{NaHCO}_{3}$ aqueous solution $(4 \times 15 \mathrm{~mL})$, dried over $\mathrm{Na}_{2} \mathrm{SO}_{4}$, filtered and concentrated under reduced pressure. The residue was purified by column chromatography [hexane-ethyl acetate (3:1, $\mathrm{v} / \mathrm{v})$ ] on silica gel to afford 17 (79.6 mg, $0.14 \mathrm{mmol}, 91 \%, \alpha: \beta=3: 7)$. Colorless solid. IR $\left(\mathrm{KBr}, \mathrm{cm}^{-1}\right)$ 3068, 3031, 2953, 2854, 2401, 2359, 1751, 1496, 1454, 1360, 1219, 1183, 1096, 1029, 946, 836, 738, 698. ${ }^{1} \mathrm{H}$ NMR $\left(\mathrm{CDCl}_{3}\right) \delta 7.31-7.18$ (m, $\left.\mathrm{CH}_{2} \underline{\mathrm{Ph}}\right), 5.78$ (dd, $\left.J=3.3 \mathrm{~Hz}, 7.7 \mathrm{~Hz}, \alpha \mathrm{H}-1\right), 5.17$ (t, $\left.J=7.4 \mathrm{~Hz}, \beta \mathrm{H}-1\right), 4.94-4.55$ (m, $\left.\underline{\mathrm{H}}_{2} \mathrm{Ph}\right), 4.36$ (d, $J=9.9 \mathrm{~Hz}$, $\alpha \mathrm{H}-5), 4.01-3.54$ (m, $\left.\alpha \mathrm{H}-2, \alpha \mathrm{H}-3, \alpha \mathrm{H}-4, \beta \mathrm{H}-2, \beta \mathrm{H}-3, \beta \mathrm{H}-4, \beta \mathrm{H}-5, \alpha \mathrm{COOC} \underline{H}_{3}, \beta \mathrm{COOC} \underline{H}_{3}, \alpha \mathrm{POC}_{3}, \beta \mathrm{POC} \underline{H}_{3}\right),-0.1$ to -1.2 (br, $\left.\underline{\mathrm{H}}_{3}\right) .{ }^{31} \mathrm{P} \mathrm{NMR}\left(\mathrm{CDCl}_{3}\right) \delta 121.6-117.8(\mathrm{~m}) .{ }^{13} \mathrm{C} \mathrm{NMR}\left(\mathrm{CDCl}_{3}\right) \delta 97.7\left(\mathrm{~d}, J_{\mathrm{C}-\mathrm{P}}=3.2 \mathrm{~Hz}, \beta \mathrm{C}-1\right), 94.4(\alpha \mathrm{C}-1)$. HRMS (ESI): calcd for $[\mathrm{M}+\mathrm{H}]^{+}: 585.2425$; found: 585.2481 .

Triethylammonium methyl 2,3,4,6-tetra- $O$-acetyl- $\beta$-D-glucopyranosyl boranophosphate (18).

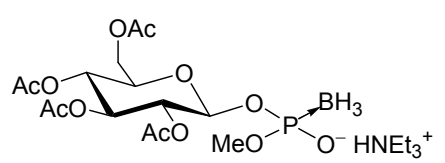

DABCO (1.69 g, $15 \mathrm{mmol})$ was added to a solution of $\mathbf{1 1} \beta(678 \mathrm{mg}, 1.5 \mathrm{mmol})$ in dry $\mathrm{CH}_{2} \mathrm{Cl}_{2}(60 \mathrm{~mL})$, and the mixture was stirred for $6 \mathrm{~h}$ at $\mathrm{rt}$. The mixture was then washed with $1 \mathrm{M}$ triethylammonium bicarbonate (TEAB) aqueous solution $(5 \times 75 \mathrm{~mL})$. The organic layer was separated, dried over $\mathrm{Na}_{2} \mathrm{SO}_{4}$, filtered and concentrated under reduced pressure. The residue was applied to a silica gel column $\left[\mathrm{CH}_{2} \mathrm{Cl}_{2}-\mathrm{MeOH}-\mathrm{Et}_{3} \mathrm{~N}(80: 10: 1, \mathrm{v} / \mathrm{v} / \mathrm{v})\right]$. The fractions containing 18 were collected, washed with $1 \mathrm{M}$ TEAB aqueous solution, dried over $\mathrm{Na}_{2} \mathrm{SO}_{4}$, filtered, and concentrated to dryness to afford 18 as a mixture of $P$-diastereoisomers (743 mg, $1.4 \mathrm{mmol}, 92 \%)$. Colorless solid. IR $\left(\mathrm{KBr}, \mathrm{cm}^{-1}\right)$ 3420, 2984, 2949, 2639, 2366, 1751, 1376, 1227, 1150, 1118, 1062, 1037, 910, 806. ${ }^{1} \mathrm{H}$ NMR $\left(\mathrm{CDCl}_{3}\right) \delta 12.43$ (br, $1 \mathrm{H}$, $\mathrm{NH}^{+}$), 5.27-4.97 (m, 4H, H-1-H-4), 4.27-4.08 (m, 2H, H-6), 3.79-3.74 (m, 1H, H-5), 3.54 (dd, 3H, J=10.7Hz, 14.3Hz, $\mathrm{POCH}_{3}$ ), 3.02 (q, $6 \mathrm{H}, \mathrm{CH}_{2} \mathrm{CH}_{3}$ ), 2.07-1.99 (12H, $\left.\mathrm{COCH}_{3}\right), 1.30$ (t, $\left.9 \mathrm{H}, J=7.4 \mathrm{~Hz}, \mathrm{CH}_{2} \mathrm{C}_{3}\right), 1.2$ to -0.2 (br, $3 \mathrm{H}, \mathrm{BH}_{3}$ ). ${ }^{31} \mathrm{P}$ NMR $\left(\mathrm{CDCl}_{3}\right) \delta 103.1-94.5(\mathrm{~m}) .{ }^{13} \mathrm{C}$ NMR $\left(\mathrm{CDCl}_{3}\right) \delta 170.4,170.0,169.8,169.7,169.5,169.4,169.4,94.7,94.5(\mathrm{~d}$, $\left.J_{\mathrm{C}-\mathrm{P}}=2.3 \mathrm{~Hz}\right), 73.0,72.9,72.0,71.9,71.8,68.6,68.4,62.2,61.8,50.5\left(\mathrm{~d}, J_{\mathrm{C}-\mathrm{P}}=5.2 \mathrm{~Hz}\right), 49.9\left(\mathrm{~d}, J_{\mathrm{C}-\mathrm{P}}=6.9 \mathrm{~Hz}\right), 45.4$, 21.1, 20.9, 20.9, 20.8, 20.7, 8.6. HRMS (ESI): calcd for $[\mathrm{M}+2 \mathrm{H}]^{+}: 439.1174$; found: 439.1175 .

Methyl 2,3,4,6-tetra- $O$-acetyl- $\beta$-D-glucopyranosyl $N$-tert-butyl-phosphoramidate (20).

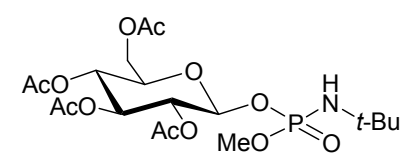

A solution of $18(135 \mathrm{mg}, 0.25 \mathrm{mmol})$ and $\mathrm{TrOMe}(206 \mathrm{mg}, 0.75 \mathrm{mmol})$ in dry $\mathrm{CH}_{2} \mathrm{Cl}_{2}(5.0 \mathrm{~mL})$ was dried over MS 4A prior to use. DCA $(100 \mu \mathrm{L}, 1.2 \mathrm{mmol})$ was added to the solution at $0{ }^{\circ} \mathrm{C}$. The mixture was kept at the same temperature for $10 \mathrm{~min}$, and $\mathrm{Et}_{3} \mathrm{SiH}(0.50 \mathrm{~mL})$ was added. The mixture was then diluted with $\mathrm{CH}_{2} \mathrm{Cl}_{2}(15 \mathrm{~mL})$, washed with $5 \% \mathrm{NaHCO}_{3}$ aqueous solution $(3 \times 15 \mathrm{~mL})$, dried over $\mathrm{Na}_{2} \mathrm{SO}_{4}$, filtered, and concentrated under reduced pressure. 
${ }^{1} \mathrm{H}$ and ${ }^{31} \mathrm{P}$ NMR spectra of the crude product clearly showed the quantitative formation of the $H$-phosphonate intermediate 19, which was used for the next step without further purification. A solution of tert-butylamine $(150 \mu \mathrm{L}$, $1.4 \mathrm{mmol})$ and $\mathrm{CCl}_{4}(250 \mu \mathrm{L}, 2.6 \mathrm{mmol})$ in dry $\mathrm{CH}_{3} \mathrm{CN}(5.0 \mathrm{~mL})$ was added dropwise to crude 19 at $0{ }^{\circ} \mathrm{C}$. The mixture was then stirred for $1 \mathrm{~h}$ at $\mathrm{rt}$ and diluted with $5 \% \mathrm{NaHCO}_{3}$ aqueous solution $(3 \mathrm{~mL})$ and $\mathrm{CH}_{2} \mathrm{Cl}_{2}(25 \mathrm{~mL})$. The organic layer was separated, washed with $5 \% \mathrm{NaHCO}_{3}$ aqueous solution $(3 \times 15 \mathrm{~mL})$, dried over $\mathrm{Na}_{2} \mathrm{SO}_{4}$, filtered and concentrated under reduced pressure. The residue was purified by silica gel column chromatography [ethyl acetate-triethylamine $(100: 1, \mathrm{v} / \mathrm{v})]$ to afford $\mathbf{2 0}$ as a mixture of $P$-diastereoisomers $(99.7 \mathrm{mg}, 0.20 \mathrm{mmol}, 80 \%)$. Colorless solid. IR $\left(\mathrm{KBr}, \mathrm{cm}^{-1}\right)$ 3478, 3347, 3218, 2970, 1756, 1432, 1368, 1229, 1162, 1088, 1036, 911, 880, 853. ${ }^{1} \mathrm{H}$ NMR (CDCl $)$ $)$ 5.32-5.04 (m, 4H, H-1-H-4), 4.28-4.14 (m, 2H, H-6), 3.85-3.80 (m, 1H, H-5), 3.68 (dd, 3H, $J=11.6$ $\left.\mathrm{Hz}, 16.5 \mathrm{~Hz}, \mathrm{POC}_{3}\right), 2.81$ (d, $\left.J=8.3 \mathrm{~Hz}, \mathrm{~N} \underline{\mathrm{H}}\right), 2.75$ (d, $\left.J=7.7 \mathrm{~Hz}, \mathrm{~N} \underline{\mathrm{H}}\right), 2.08-2.00$ (m, 12H, COC$\left.\underline{H}_{3}\right), 1.27$ (s, tert-Bu), $1.26\left(\mathrm{~s}\right.$, tert-Bu) ${ }^{31} \mathrm{P}$ NMR $\left(\mathrm{CDCl}_{3}\right) \delta 7.9,7.4 .{ }^{13} \mathrm{C} \mathrm{NMR}\left(\mathrm{CDCl}_{3}\right) \delta 170.2,169.8,169.7,169.2,169.0,95.3\left(\mathrm{~d}, J_{\mathrm{C}-\mathrm{P}}=4.6\right.$ $\mathrm{Hz}), 95.2\left(\mathrm{~d}, J_{\mathrm{C}-\mathrm{P}}=5.8\right), 72.6,72.5,72.3,72.3,71.3,71.3,71.2,71.1,67.9,67.9,61.6,61.5,53.2\left(\mathrm{~d}, J_{\mathrm{C}-\mathrm{P}}=1.4 \mathrm{~Hz}\right), 53.1$ $\left(\mathrm{d}, J_{\mathrm{C}-\mathrm{P}}=1.1 \mathrm{~Hz}\right), 51.2,51.0,31.1,31.1,31.1,31.0,20.7,20.7,20.7,20.6,20.6$. HRMS (ESI): calcd for [M+Na] ${ }^{+}$: 520.1554; found: 520.1554 .

Triethylammonium methyl 2,3,4,6-tetra- $O$-acetyl- $\beta$-D-glucopyranosyl phosphorothioate (21).

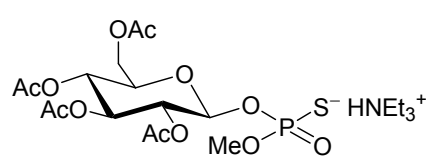

Crude $H$-phosphonate intermediate 19 obtained as above was diluted with dry pyridine $(1.0 \mathrm{~mL}) . \mathrm{S}_{8}(256 \mathrm{mg}, 1.0$ mmol) was added to the solution, and the reaction mixture was stirred for $2 \mathrm{~h}$ at rt. An excess of $\mathrm{S}_{8}$ was filtered off and the filtrate was concentrated under reduced pressure. The residue was applied to a silica gel column $\left[\mathrm{CH}_{2} \mathrm{Cl}_{2}-\mathrm{MeOH}-\mathrm{Et}_{3} \mathrm{~N}(90: 10: 1, \mathrm{v} / \mathrm{v} / \mathrm{v})\right]$. The fractions containing 21 were collected, washed with $1 \mathrm{M}$ TEAB aqueous solution, dried over $\mathrm{Na}_{2} \mathrm{SO}_{4}$, filtered, and concentrated to dryness to afford 21 as a mixture of $P$-diastereoisomers (75.1 mg, 0.13 mmol, 54\%). Colorless oil. IR (KBr, cm $\left.{ }^{-1}\right)$ 3434, 2986, 2947, 2682, 2441, 1753, 1368, 1229, 1155, 1038, 911 , 815, 783. ${ }^{1} \mathrm{H} \mathrm{NMR}\left(\mathrm{CDCl}_{3}\right) \delta 11.9\left(\mathrm{br}, 1 \mathrm{H}, \mathrm{NH}^{+}\right), 5.47(\mathrm{dd}, J=8.0 \mathrm{~Hz}, 11.0 \mathrm{~Hz}, \mathrm{H}-1), 5.39(\mathrm{dd}, J=8.0 \mathrm{~Hz}, 11.3 \mathrm{~Hz}$, H-1), 5.25 (t, $J=9.5 \mathrm{~Hz}, \mathrm{H}-3$ ), 5.24 (t, $J=9.5 \mathrm{~Hz}, \mathrm{H}-3$ ), 5.13-5.01 (m, 2H, H-2, H-4), 4.27-4.09 (m, 2H, H-6), 3.84-3.79 (m, 1H, H-5), 3.58 (dd, $\left.3 \mathrm{H}, J=13.5 \mathrm{~Hz}, 14.6 \mathrm{~Hz}, \mathrm{POC}_{3}\right), 3.07$ (q, 6H, $\left.\mathrm{CH}_{2} \mathrm{CH}_{3}\right), 2.06-1.99$ (m, 12H, $\left.\mathrm{COC}_{3}\right), 1.32\left(\mathrm{t}, 9 \mathrm{H}, J=7.4 \mathrm{~Hz}, \mathrm{CH}_{2} \mathrm{CH}_{3}\right) .{ }^{31} \mathrm{P} \mathrm{NMR}\left(\mathrm{CDCl}_{3}\right) \delta 59.2,59.1 .{ }^{13} \mathrm{C} \mathrm{NMR}\left(\mathrm{CDCl}_{3}\right) \delta 170.7,170.7,170.2$, $170.1,169.8,169.7,169.7,169.6,96.7\left(\mathrm{~d}, J_{\mathrm{C}-\mathrm{P}}=3.7 \mathrm{~Hz}\right), 96.4\left(\mathrm{~d}, J_{\mathrm{C}-\mathrm{P}}=4.6 \mathrm{~Hz}\right), 73.3,72.3,72.2,72.1,72.1,71.8,71.7$, 68.8, 68.6, 62.4, 62.0, $53.60\left(\mathrm{~d}, J_{\mathrm{C}-\mathrm{P}}=6.6 \mathrm{~Hz}\right), 53.5\left(\mathrm{~d}, J_{\mathrm{C}-\mathrm{P}}=6.0 \mathrm{~Hz}\right), 45.9,21.4,21.1,21.1,21.1,21.0,8.9 . \mathrm{HRMS}$ (ESI): calcd for [M] $:$ : 457.0575; found: 457.0575 .

Methyl ( $S$ )- $\beta$-citronellyl 2,3,4,6-tetra- $O$-acetyl- $\beta$-D-glucopyranosyl boranophosphate (22).

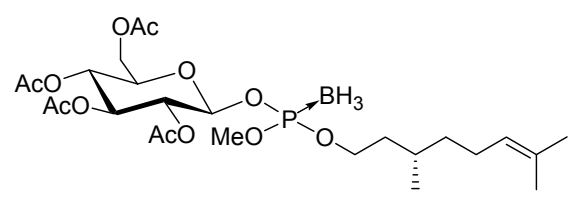

NT (43.4 mg, $0.38 \mathrm{mmol})$ and Bop-Cl $(96.0$ g. $0.38 \mathrm{mmol})$ were successively added to a solution of $\mathbf{1 8}$ (81.6 mg, $0.15 \mathrm{mmol}), i-\mathrm{Pr}_{2} \mathrm{NEt}(0.26 \mathrm{~mL}, 1.5 \mathrm{mmol})$ and $(S)$ - $\beta$-citronellol $(26.1 \mathrm{mg}, 0.17 \mathrm{mmol})$ in dry THF $(1.5 \mathrm{~mL})$, and the mixture was stirred for $1 \mathrm{~h}$ at $\mathrm{rt}$. The mixture was diluted with saturated $\mathrm{NaHCO}_{3}$ aqueous solution $(5 \mathrm{~mL})$ and $\mathrm{CH}_{2} \mathrm{Cl}_{2}$ $(15 \mathrm{~mL})$. The organic layer was separated, washed with saturated $\mathrm{NaHCO}_{3}$ aqueous solution $(4 \times 30 \mathrm{~mL})$, dried over $\mathrm{Na}_{2} \mathrm{SO}_{4}$, filtered, and concentrated under reduced pressure. The residue was purified by silica gel column 
chromatography [hexane-acetone $(13: 7, \mathrm{v} / \mathrm{v})]$ to afford 22 as a mixture of $P$-diastereoisomers $(81.3 \mathrm{mg}, 0.14 \mathrm{mmol}$, 96\%). Colorless oil. IR $\left(\mathrm{KBr}, \mathrm{cm}^{-1}\right)$ 2961, 2917, 2863, 2403, 2357, 1757, 1437, 1368, 1225, 1037, 909, 855, 830. ${ }^{1} \mathrm{H}$ NMR $\left(\mathrm{CDCl}_{3}\right) \delta 5.27-5.05(\mathrm{~m}, 5 \mathrm{H}), 4.28-3.97(\mathrm{~m}, 4 \mathrm{H}), 3.82-3.78(\mathrm{~m}, 1 \mathrm{H}, \mathrm{H}-5), 3.70\left(\mathrm{t}, 3 \mathrm{H}, J=11.3 \mathrm{~Hz}, \mathrm{POC}_{3}\right)$ 2.08-1.13 (m, 25H), 0.94 (d, 3H, $J=6.3 \mathrm{~Hz}$ ), 1.2 to -0.2 (br, $\left.3 \mathrm{H}, \mathrm{BH}_{3}\right) .{ }^{31} \mathrm{P}$ NMR $\left(\mathrm{CDCl}_{3}\right) \oint 120.2-115.2(\mathrm{~m}) .{ }^{13} \mathrm{C}$ NMR $\left(\mathrm{CDCl}_{3}\right) \delta 170.3,169.8,169.2,169.1,169.0,131.3,124.3,124.2,95.7,95.6,72.6,72.3,71.3,71.2,68.0,67.9$, $66.0\left(\mathrm{~d}, J_{\mathrm{C}-\mathrm{P}}=4.3 \mathrm{~Hz}\right), 65.9\left(\mathrm{~d}, J_{\mathrm{C}-\mathrm{P}}=4.9 \mathrm{~Hz}\right), 61.5,53.3\left(\mathrm{~d}, J_{\mathrm{C}-\mathrm{P}}=5.5 \mathrm{~Hz}\right), 37.1,37.0,37.0,36.9,36.8,29.0,25.8,25.4$, 20.7, 20.6, 19.3, 19.2, 17.7. HRMS (ESI): calcd for [M-H] $]^{+}$577.2584; found: 577.2570.

Triethylammonium (S)- $\beta$-citronellyl 2,3,4,6-tetra- $O$-acetyl- $\beta$-D-glucopyranosyl boranophosphate (23).

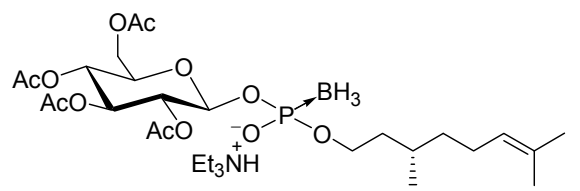

Benzenethiol $(265 \mu \mathrm{L}, 2.6 \mathrm{mmol})$ and triethylamine $(360 \mu \mathrm{L}, 2.6 \mathrm{mmol})$ were added to a solution of $22(73.2 \mathrm{mg}$, $0.13 \mathrm{mmol})$ in dry THF $(1.3 \mathrm{~mL})$. The mixture was stirred for $20 \mathrm{~h}$ at $\mathrm{rt}$, and concentrated to dryness. The residue was purified by silica gel column chromatography $\left[\mathrm{CH}_{2} \mathrm{Cl}_{2}-\mathrm{MeOH}-\mathrm{Et}_{3} \mathrm{~N}(98.5: 0.5: 1, \mathrm{v} / \mathrm{v} / \mathrm{v})\right]$ to afford 23 as a mixture of $P$-diastereoisomers (83.7 mg, $0.13 \mathrm{mmol}, 97 \%)$. Colorless solid. IR $\left(\mathrm{KBr}, \mathrm{cm}^{-1}\right)$ 2959, 2917, 2863, 2364, 1754, 1451, 1377, 1226, 1061, 908, 808. ${ }^{1} \mathrm{H}$ NMR $\left(\mathrm{CDCl}_{3}\right) \delta 12.51$ (br, 1H, NH$\left.{ }^{+}\right), 5.26-4.96(\mathrm{~m}, 5 \mathrm{H}), 4.28-4.07$ (m, 2H, H-6), 4.01-3.73 (m, 3H), 3.02 (q, 6H, $\underline{\mathrm{CH}}_{2} \mathrm{CH}_{3}$ ), 2.06-1.07 (m, 34H), 0.89 (d, $\left.3 \mathrm{H}, J=1.9 \mathrm{~Hz}\right), 1.2$ to -0.2 (br, $\left.3 \mathrm{H}, \mathrm{BH}_{3}\right) .{ }^{31} \mathrm{P}$ $\operatorname{NMR}\left(\mathrm{CDCl}_{3}\right) \delta 100.2-93.5(\mathrm{~m}) .{ }^{13} \mathrm{C} \mathrm{NMR}\left(\mathrm{CDCl}_{3}\right) \delta 170.4,170.4,169.9,169.8,169.6,169.39,169.35,169.3,130.9$, 124.7, 124.6, 94.6, 73.0, 71.9, 68.4, 62.2, 62.1, 62.0, 61.8, 61.7, 61.6, 45.3, 37.9, 37.8, 37.2, 37.1, 29.4, 29.3, 25.8, 25.5, 21.1, 20.8, 20.7, 19.5, 19.4, 17.7, 8.5. HRMS (ESI): calcd for [M]': 563.2438; found: 563.2418 .

Triethylammonium ( $S$ )- $\beta$-citronellyl $\beta$-D-glucopyranosyl boranophosphate (24).

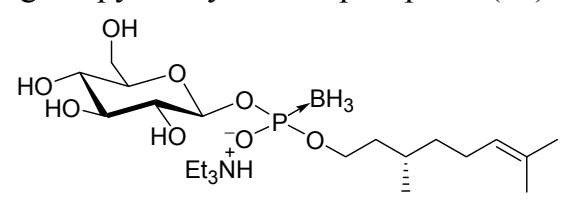

Compound $23(73.4 \mathrm{mg}, 0.11 \mathrm{mmol})$ was treated with $25 \%$ aqueous ammonia $(2.2 \mathrm{~mL})$ for $1 \mathrm{~h}$ at $\mathrm{rt} .1 \mathrm{M}$ TEAB aqueous solution $(1.0 \mathrm{~mL})$ was added, and the mixture was concentrated under reduced pressure. The residue was purified by Sephadex LH-20 column chromatography $\left[\mathrm{MeOH}-\mathrm{Et}_{3} \mathrm{~N}(99: 1, \mathrm{v} / \mathrm{v})\right]$ to afford 24 as a mixture of $P$-diastereomers (50.9 mg, $0.10 \mathrm{mmol}, 93 \%)$. Colorless foam. IR $\left(\mathrm{KBr}, \mathrm{cm}^{-1}\right) 3389,2956,2921,2863,2695,2490,2368$, 1640, 1452, 1379, 1132, 1064, 901, 823. ${ }^{1} \mathrm{H}$ NMR $\left(\mathrm{CD}_{3} \mathrm{CN}\right) \delta 5.13-5.07$ (m, 1H, allyl), 4.90-4.83 (m, 1H, H-1), 3.95-3.80 (m, 2H), 3.79-3.50 (m, 2H, H-6), 3.38-3.10 (m, 4H, H-2, H-3, H-4, H-5), 3.05 (q, 6H, $\left.\mathrm{CH}_{2} \mathrm{CH}_{3}\right), 1.98$ (m, 1H), $1.67-1.08(\mathrm{~m}, 21 \mathrm{H}), 0.89(\mathrm{~d}, 3 \mathrm{H}, J=6.6 \mathrm{~Hz}), 1.2$ to $-0.2\left(\mathrm{br}, 3 \mathrm{H}, \mathrm{BH}_{3}\right) .{ }^{31} \mathrm{P} \mathrm{NMR}\left(\mathrm{CD}_{3} \mathrm{CN}\right) \delta 97.5-91.5(\mathrm{~m}) .{ }^{13} \mathrm{C}$ $\left(\mathrm{CD}_{3} \mathrm{CN}\right)$ NMR $\delta 131.6,125.4,97.0\left(\mathrm{~d} J_{\mathrm{C}-\mathrm{P}}=3.2 \mathrm{~Hz}\right), 96.6\left(\mathrm{~d}, J_{\mathrm{C}-\mathrm{P}}=7.2 \mathrm{~Hz}\right), 77.5,77.5,77.3,77.3\left(\mathrm{~d}, J_{\mathrm{C}-\mathrm{P}}=4.0 \mathrm{~Hz}\right)$, $75.2\left(\mathrm{~d}, J_{\mathrm{C}-\mathrm{P}}=3.5 \mathrm{~Hz}\right), 71.0,70.8,62.8\left(\mathrm{~d}, J_{\mathrm{C}-\mathrm{P}}=3.7 \mathrm{~Hz}\right), 62.5,62.3,62.2\left(\mathrm{~d}, J_{\mathrm{C}-\mathrm{P}}=4.9 \mathrm{~Hz}\right), 46.6,38.4,38.3,37.7,37.7$, 29.9, 29.9, 26.0, 26.0, 25.7, 19.6, 17.7, 8.9. HRMS (ESI): calcd for [M] $]^{-}: 345.2015$; found: 345.2015.

Dimethyl 2,3,4,6-tetra- $O$-acetyl- $\beta$-D-glucopyranosyl phosphate (26).

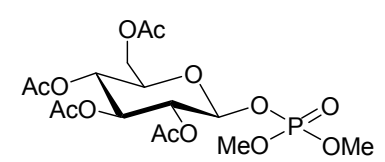


To a solution of $\mathbf{1 1} \beta(90.6 \mathrm{mg}, 0.20 \mathrm{mmol})$ in dry $\mathrm{CH}_{2} \mathrm{Cl}_{2}(1.0 \mathrm{~mL})$ was added $m \mathrm{CPBA}(344 \mathrm{mg}, 2.0 \mathrm{mmol})$ portionwise at $\mathrm{rt}$. The mixture was stirred for $10 \mathrm{~min}$ at the same temperature. The mixture was diluted with $10 \%$ sodium thiosulfate aqueous solution $(10 \mathrm{~mL})$ and $\mathrm{CH}_{2} \mathrm{Cl}_{2}(15 \mathrm{~mL})$. The separated organic layer was washed with saturated $\mathrm{NaHCO}_{3}$ aqueous solution $(5 \times 15 \mathrm{~mL})$, dried over $\mathrm{Na}_{2} \mathrm{SO}_{4}$, filtered and concentrated under reduced pressure. The residue was purified by a short silica gel column chromatography (ethyl acetate) to afford $\mathbf{2 6}$ ( $82.3 \mathrm{mg}, 0.18 \mathrm{mmol}$, 90\%). Colorless solid. IR (KBr, $\left.\mathrm{cm}^{-1}\right) 2962,2862,1754,1439,1369,1221,1094,1035,881 .{ }^{1} \mathrm{H} \mathrm{NMR}\left(\mathrm{CDCl}_{3}\right) \delta 5.28(\mathrm{t}$, $J=7.6 \mathrm{~Hz}, 1 \mathrm{H}, \mathrm{H}-1), 5.22$ (t, $J=9.4 \mathrm{~Hz}, 1 \mathrm{H}, \mathrm{H}-3), 5.14-5.06$ (m, 2H, H-2, H-4), 4.27 (dd, 1H, $J=5.0 \mathrm{~Hz}, 12.4 \mathrm{~Hz}$, H-6), 4.16 (dd, 1H, J = 2,2 Hz, 12.4 Hz, H-6), 3.85-3.80 (m, 1H, H-5), 3.78 (d, 3H, J=11.6 Hz, POC $\left.\underline{H}_{3}\right), 3.74$ (d, $3 \mathrm{H}$, $\left.J=11.3 \mathrm{~Hz}, \mathrm{POC}_{3}\right), 2.08\left(\mathrm{~s}, 3 \mathrm{H}, \mathrm{COC} \underline{H}_{3}\right), 2.07\left(\mathrm{~s}, 3 \mathrm{H}, \mathrm{COC} \underline{H}_{3}\right), 2.04\left(\mathrm{~s}, 3 \mathrm{H}, \mathrm{COC} \underline{H}_{3}\right), 2.01\left(\mathrm{~s}, 3 \mathrm{H}, \mathrm{COC} \underline{H}_{3}\right) .{ }^{31} \mathrm{P}$ $\operatorname{NMR}\left(\mathrm{CDCl}_{3}\right) \delta-0.30 .{ }^{13} \mathrm{C} \mathrm{NMR}\left(\mathrm{CDCl}_{3}\right) \delta 170.3,169.8,169.2,169.1,96.1\left(\mathrm{~d}, J_{\mathrm{C}-\mathrm{P}}=4.6 \mathrm{~Hz}\right), 72.6,72.2\left(\mathrm{~d}, J_{\mathrm{C}-\mathrm{P}}=1.7\right.$ $\mathrm{Hz}), 71.1\left(\mathrm{~d}, J_{\mathrm{C}-\mathrm{P}}=8.9 \mathrm{~Hz}\right), 67.8,61.5,54.7\left(\mathrm{~d}, J_{\mathrm{C}-\mathrm{P}}=1.4 \mathrm{~Hz}\right), 54.6\left(\mathrm{~d}, J_{\mathrm{C}-\mathrm{P}}=1.2 \mathrm{~Hz}\right), 20.8,20.6$. HRMS (ESI): calcd for $[\mathrm{M}+\mathrm{Na}]^{+}: 479.0925$; found: 479.0903 .

Oxidation of $\mathbf{1 1} \beta$ with TBHP and DCSO were attempted by the same procedure as in the case of $m$ CPBA.

Dimethyl 2,3,4,6-tetra- $O$-benzyl-D-glucopyranosyl phosphate (25).

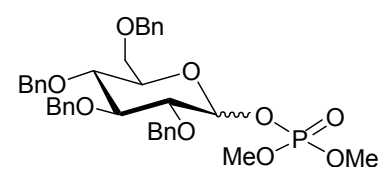

To a solution of $\mathbf{1 0}(130 \mathrm{mg}, 0.20 \mathrm{mmol})$ in dry $\mathrm{CH}_{2} \mathrm{Cl}_{2}(1.0 \mathrm{~mL})$ was added $m \mathrm{CPBA}(345 \mathrm{mg}, 2.0 \mathrm{mmol})$ portionwise at rt. The mixture was stirred for $10 \mathrm{~min}$ at the same temperature and diluted with $10 \%$ sodium thiosulfate aqueous solution $(10 \mathrm{~mL})$ and $\mathrm{CH}_{2} \mathrm{Cl}_{2}(15 \mathrm{~mL})$. The separated organic layer was washed with saturated $\mathrm{NaHCO}_{3}$ aqueous solution $(5 \times 15 \mathrm{~mL})$, dried over $\mathrm{Na}_{2} \mathrm{SO}_{4}$, filtered and concentrated under reduced pressure. The residue was purified by a short silica gel column chromatography [hexane-ethyl acetate (4:1, v/v)] to afford 25 (118 mg, $0.18 \mathrm{mmol}$, 91\%). Colorless oil. IR (KBr, cm $\left.{ }^{-1}\right)$ 3086, 3031, 3022, 2947, 2910, 2857, 1496, 1453, 1361, 1150, 1092, 1038, 959, 880, 851, 738. 698. ${ }^{1} \mathrm{H}$ NMR $\left(\mathrm{CDCl}_{3}\right) \delta 7.37-7.10\left(\mathrm{~m}, \mathrm{CH}_{2} \underline{\mathrm{Ph}}\right), 5.85(\mathrm{dd}, J=3.3 \mathrm{~Hz}, 7.7 \mathrm{~Hz}, \alpha \mathrm{H}-1), 5.14(\mathrm{t}, J=7.4 \mathrm{~Hz}$, $\beta \mathrm{H}-1), 4.96-4.44\left(\mathrm{~m}, \mathrm{C}_{2} \mathrm{Ph}\right), 3.99-3.51$ (m, $\left.\alpha \mathrm{H}-2-\alpha \mathrm{H}-6, \beta \mathrm{H}-2-\beta \mathrm{H}-6, \alpha \mathrm{POCH}_{3}, \beta \mathrm{POCH} \underline{H}_{3}\right) .{ }^{31} \mathrm{P} \mathrm{NMR}\left(\mathrm{CDCl}_{3}\right) \delta 0.7$ $(\beta), 0.7(\alpha) .{ }^{13} \mathrm{C}$ NMR $\left(\mathrm{CDCl}_{3}\right) \delta 98.8\left(\mathrm{~d}, J_{\mathrm{C}-\mathrm{P}}=6.3 \mathrm{~Hz}, \beta \mathrm{C}-1\right), 95.3\left(\mathrm{~d}, J_{\mathrm{C}-\mathrm{P}}=6.0 \mathrm{~Hz}, \alpha \mathrm{C}-1\right)$. HRMS (FAB): calcd for $[\mathrm{M}+\mathrm{Na}]^{+}:$671.2380; found: 671.2360 . 


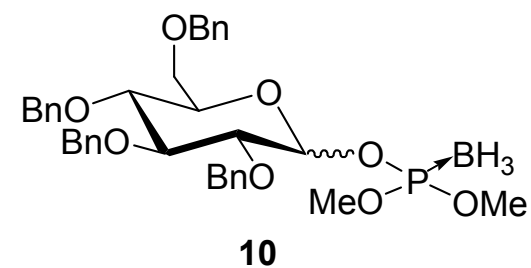

$\left(300 \mathrm{MHz}, \mathrm{CDCl}_{3}\right)$
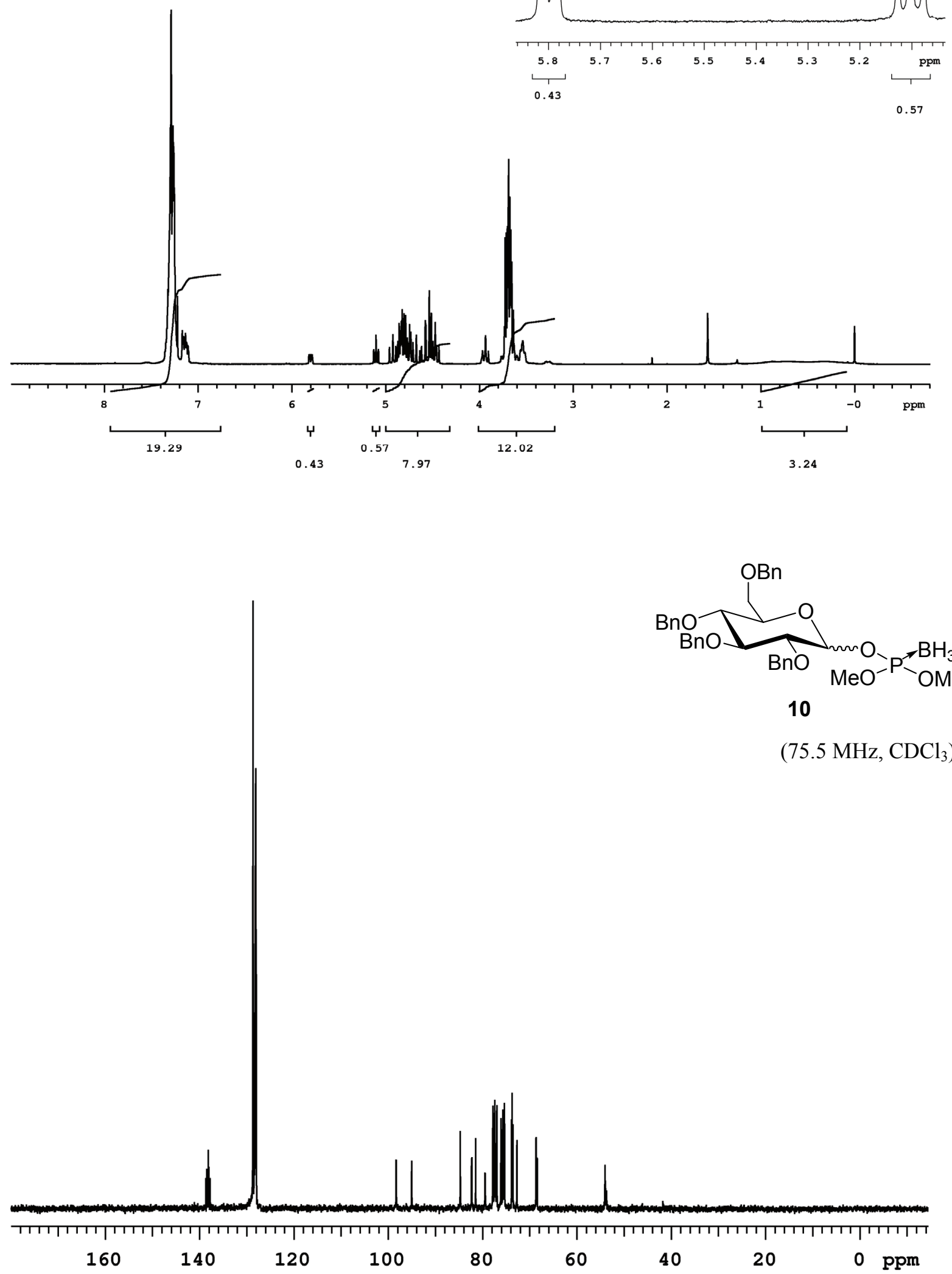


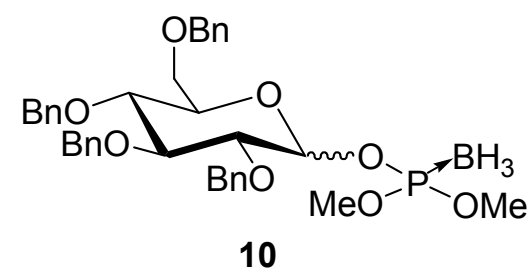

(121.5 MHz, $\left.\mathrm{CDCl}_{3}\right)$

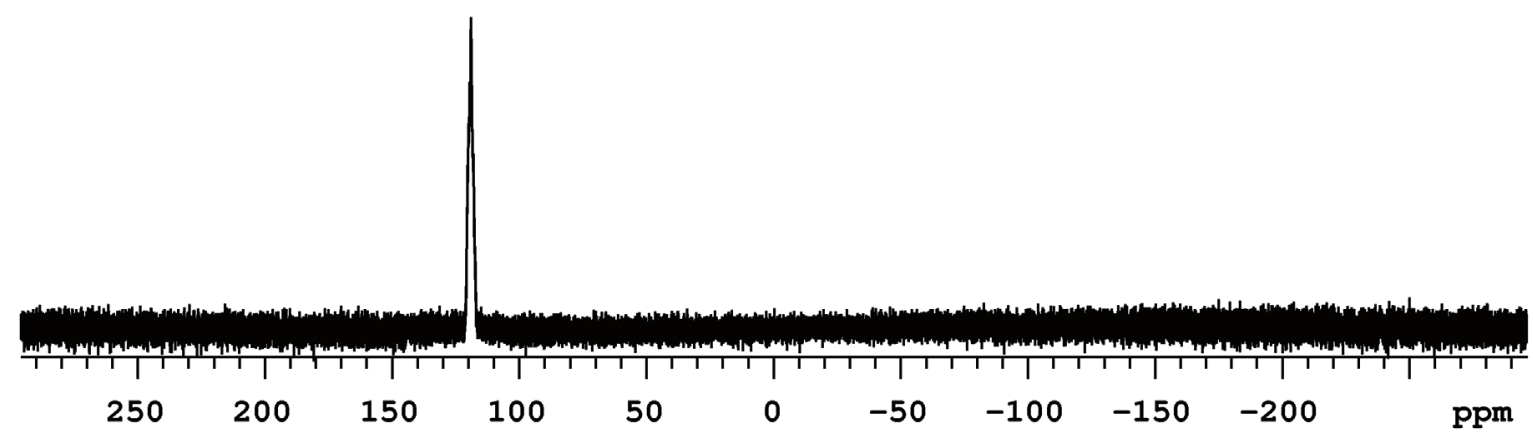

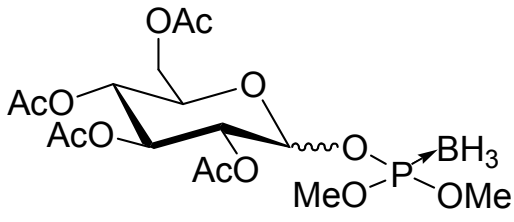

11

(300 MHz, $\mathrm{CDCl}_{3}$ )

$\alpha \mathrm{H}-2, \alpha \mathrm{H}-4$

$\beta \mathrm{H}-1, \beta \mathrm{H}-2, \beta \mathrm{H}-3, \beta \mathrm{H}-4$
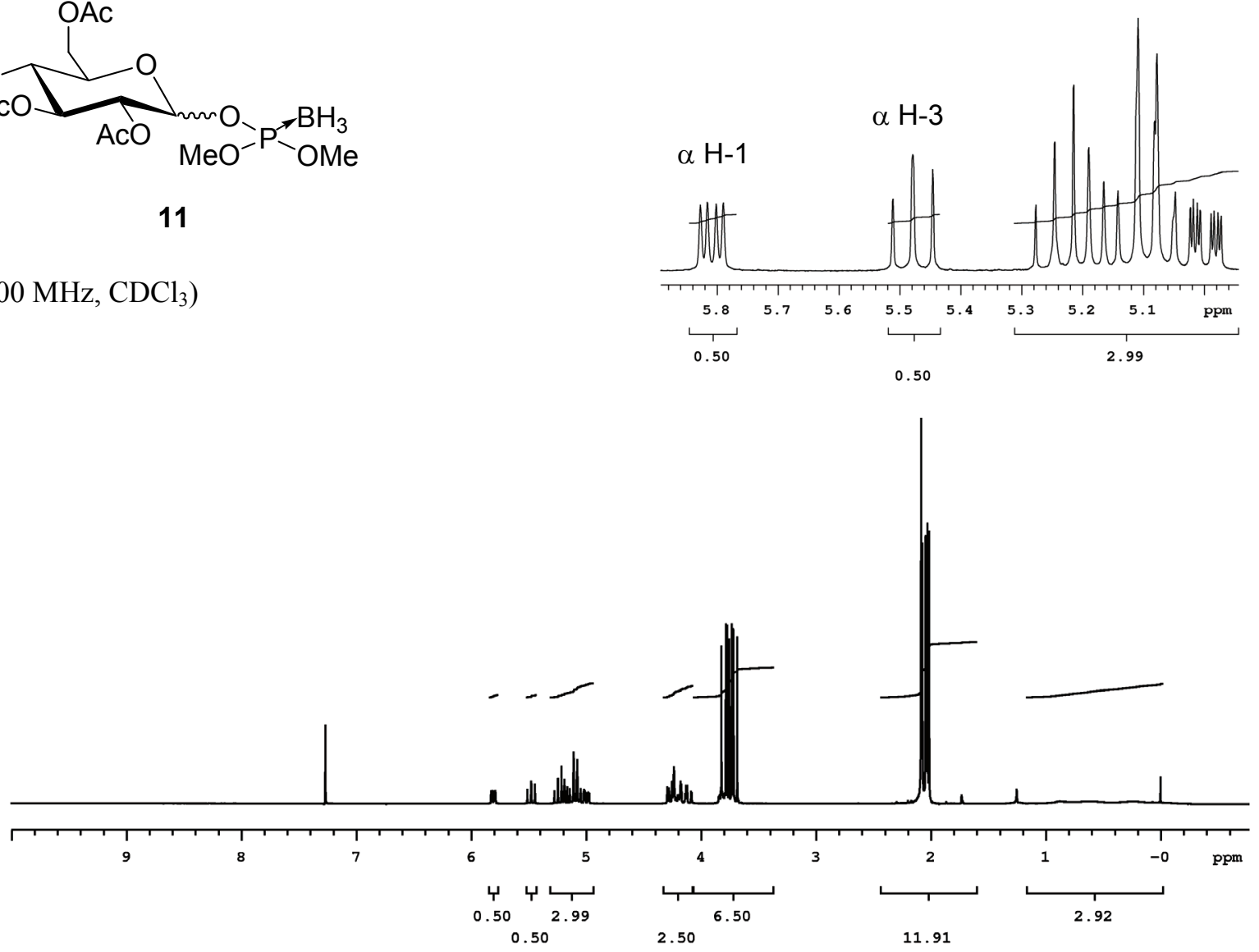


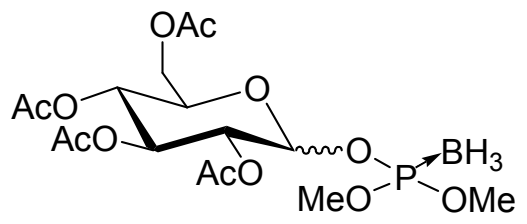

11

(75.5 MHz, $\mathrm{CDCl}_{3}$ )
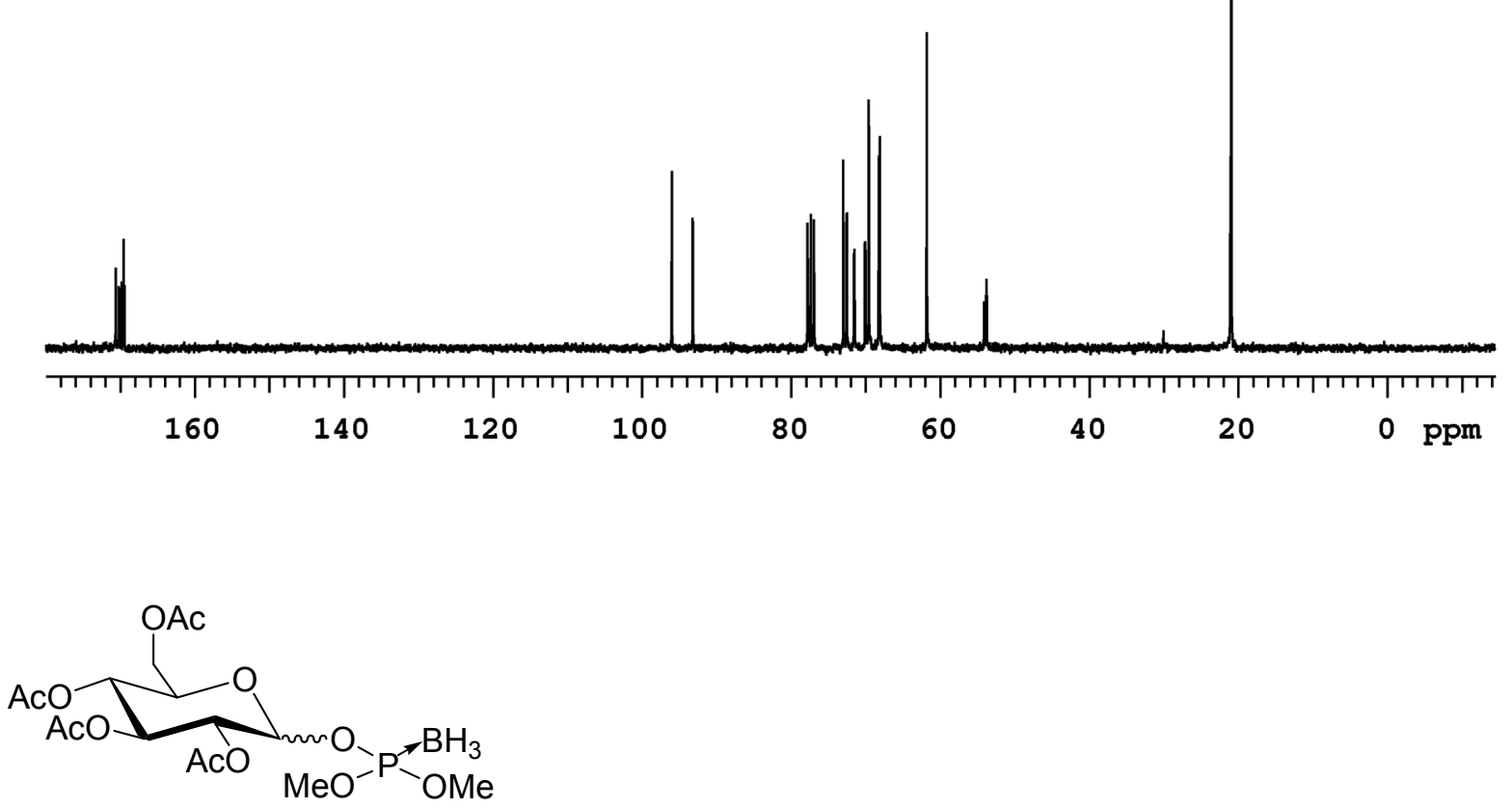

11

(121.5 MHz, $\mathrm{CDCl}_{3}$ )

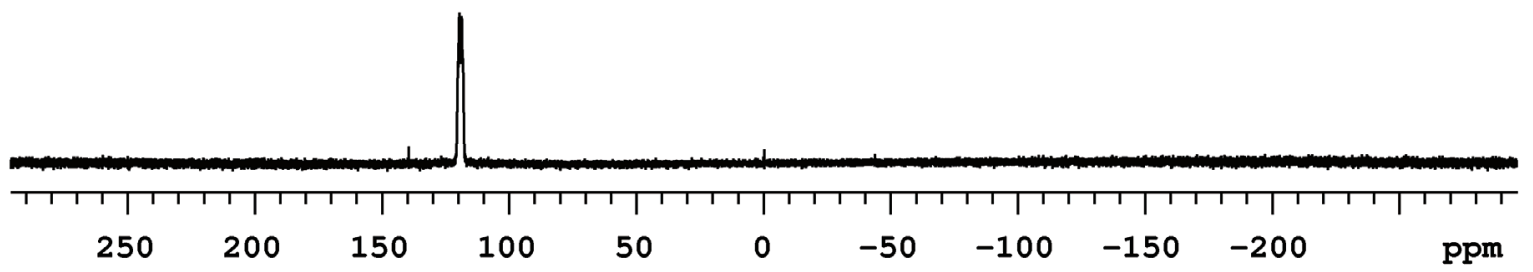

S11 
$\underbrace{\mathrm{OAc}}_{\mathrm{AcO}}$

$11 \beta$

(300 MHz, $\mathrm{CDCl}_{3}$ )

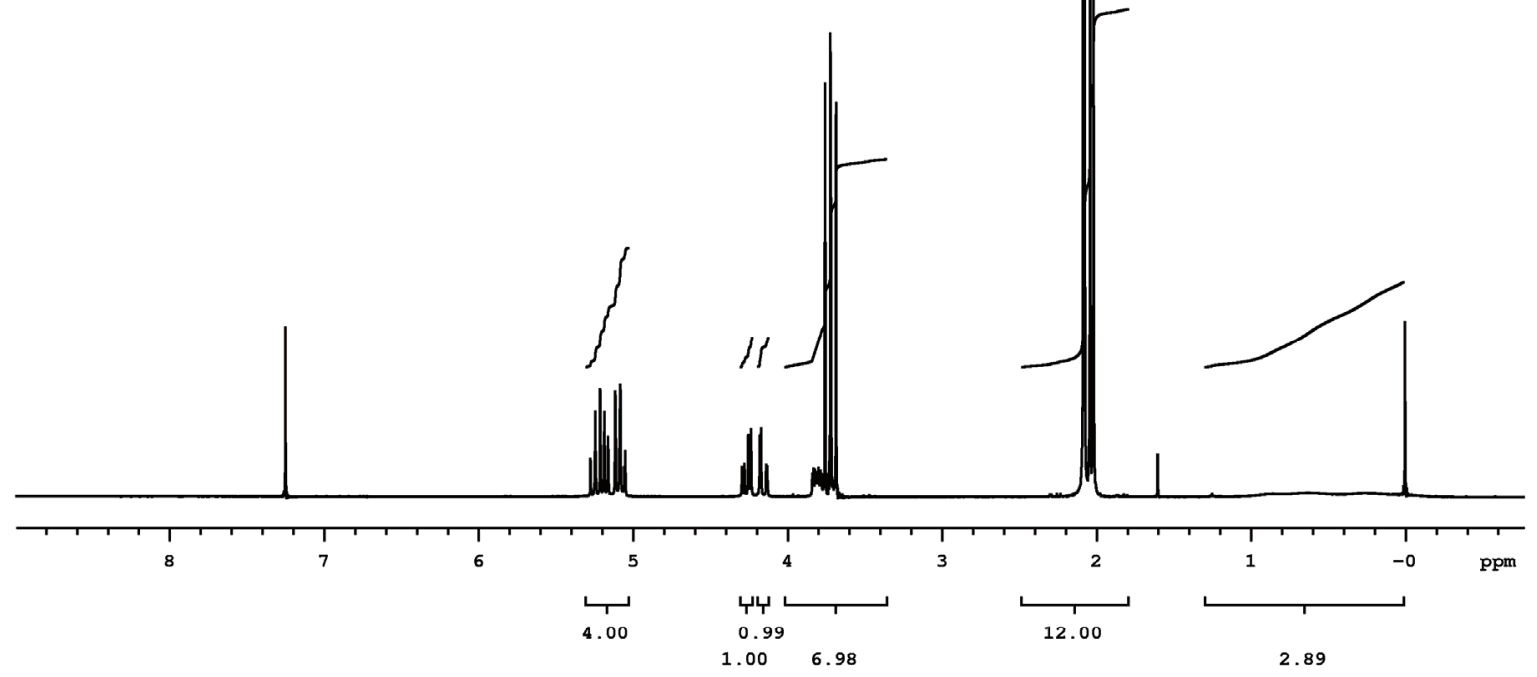

$11 \beta$

(75.5 MHz, $\mathrm{CDCl}_{3}$ )

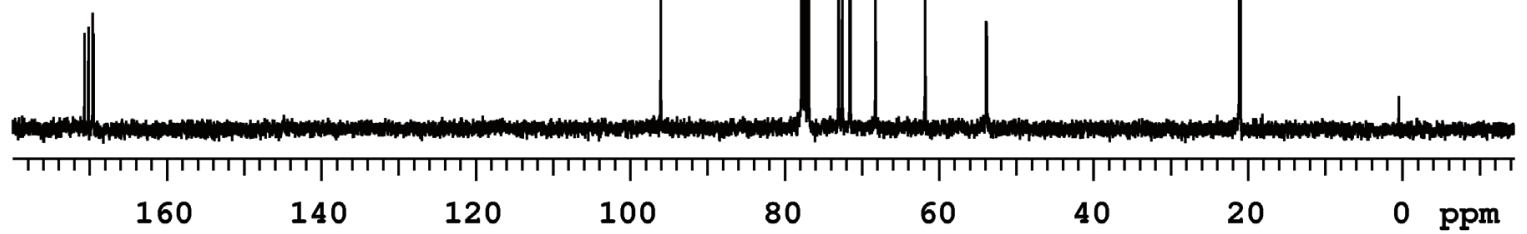




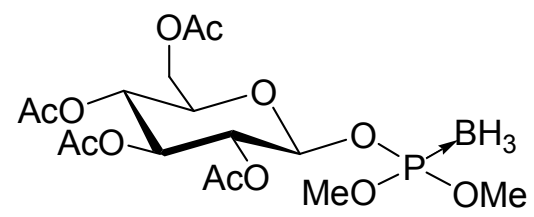

$11 \beta$

(75.5 MHz, $\mathrm{CDCl}_{3}$ )
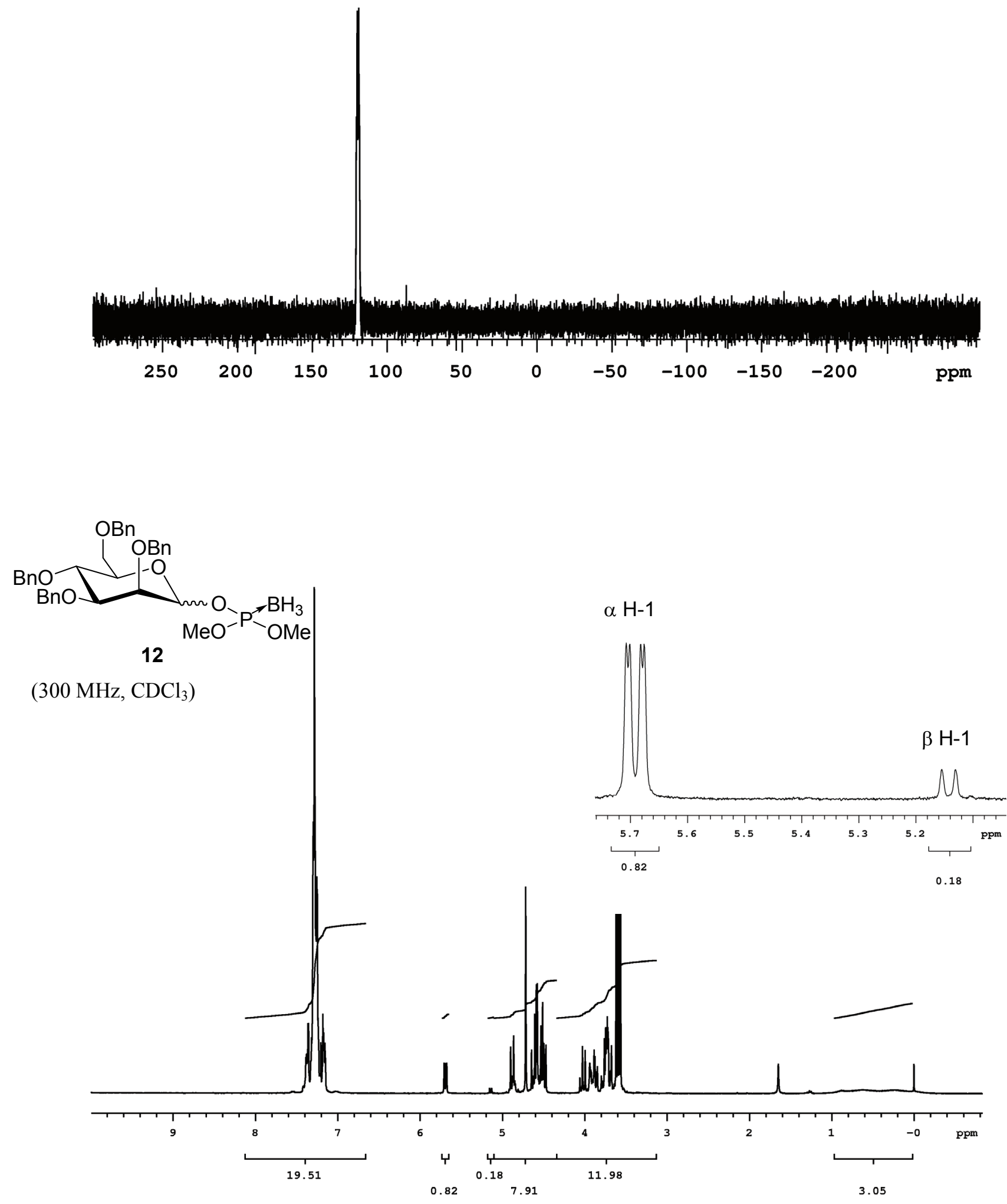


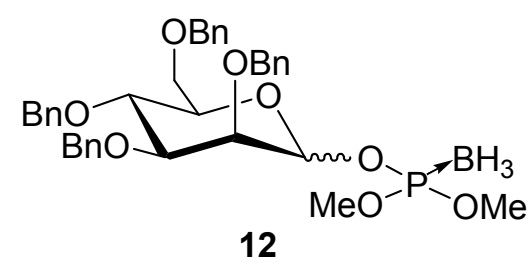

(75.5 MHz, $\mathrm{CDCl}_{3}$ )
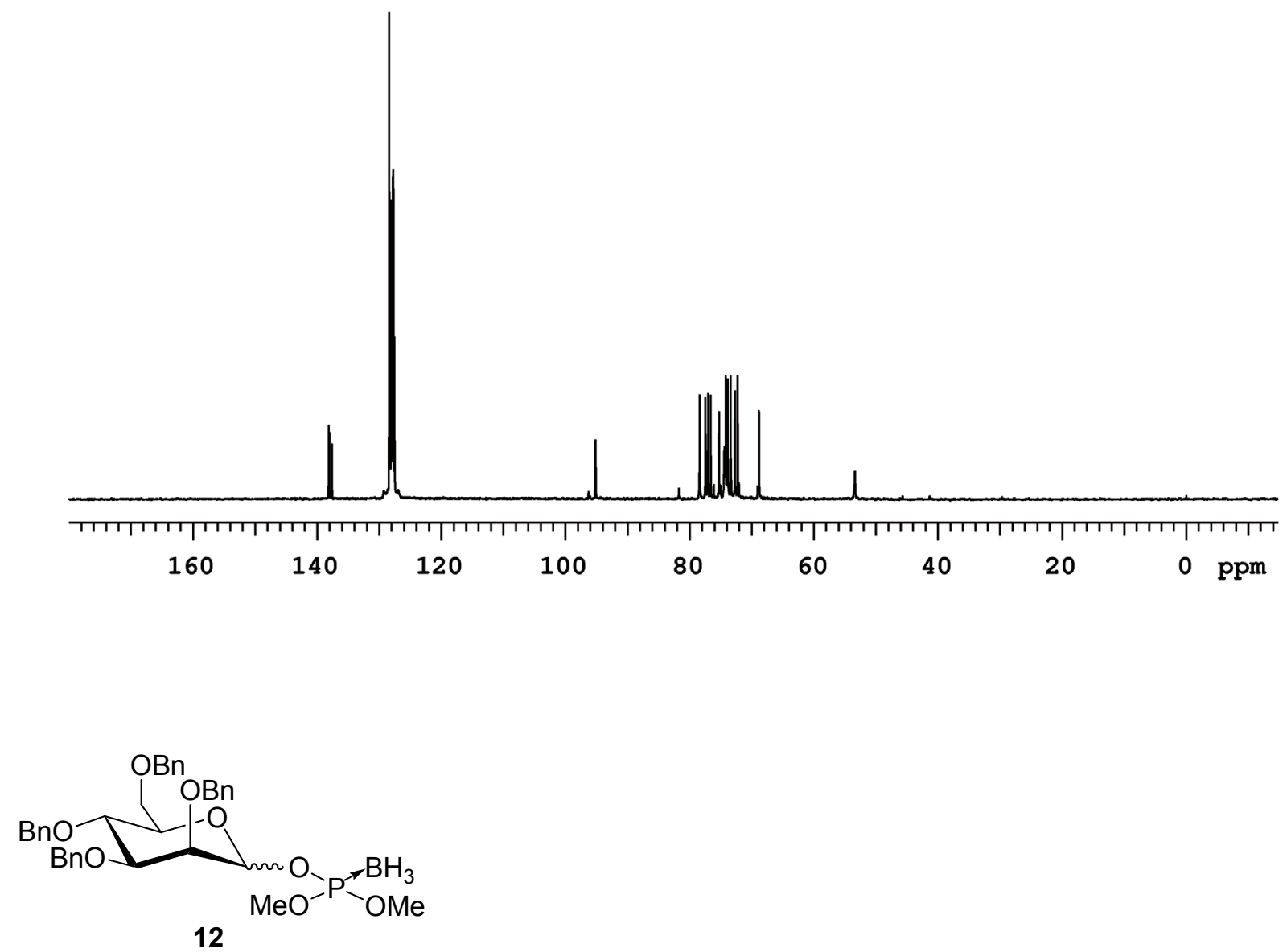

(121.5 MHz, $\mathrm{CDCl}_{3}$ )

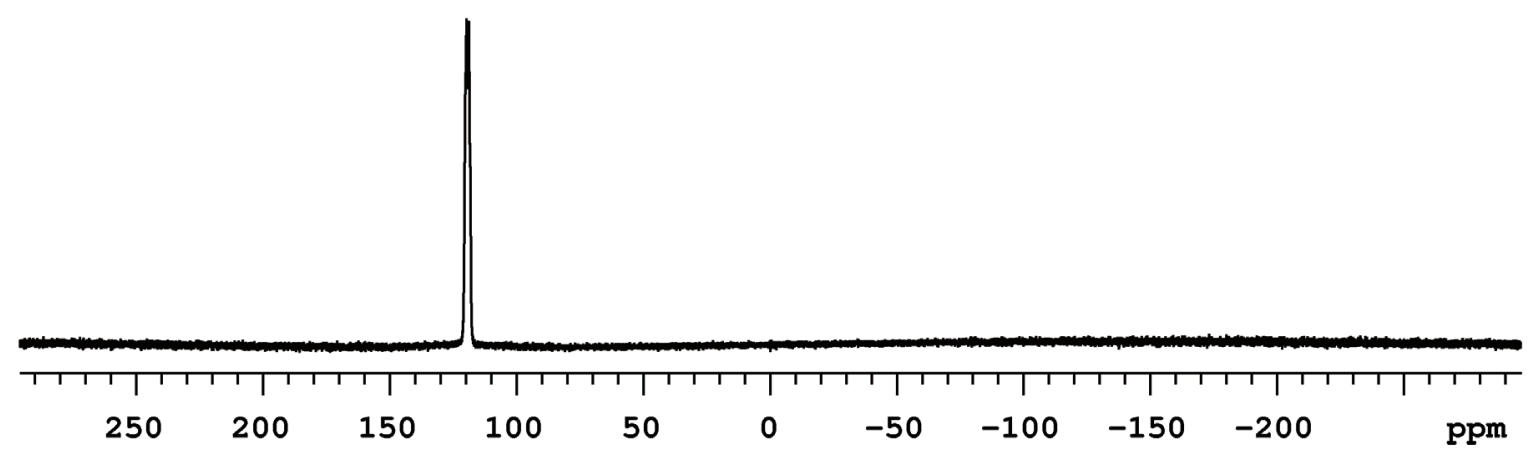




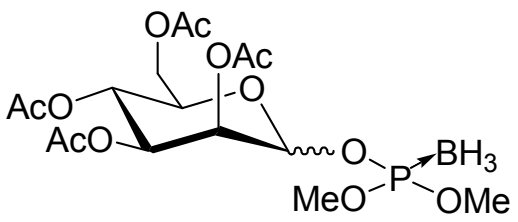

13

(300 MHz, $\mathrm{CDCl}_{3}$ )
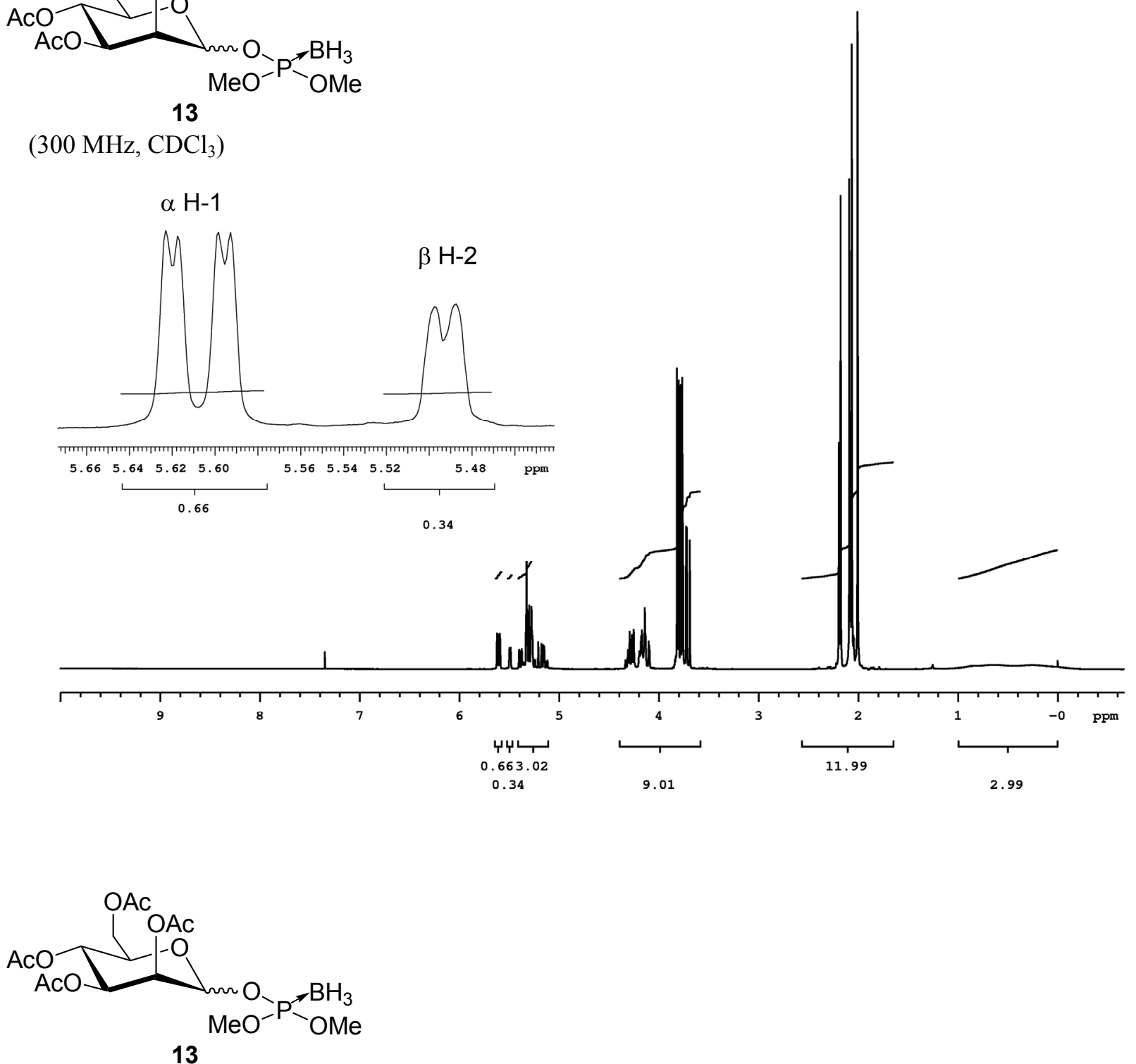

(75.5 MHz, $\mathrm{CDCl}_{3}$ )

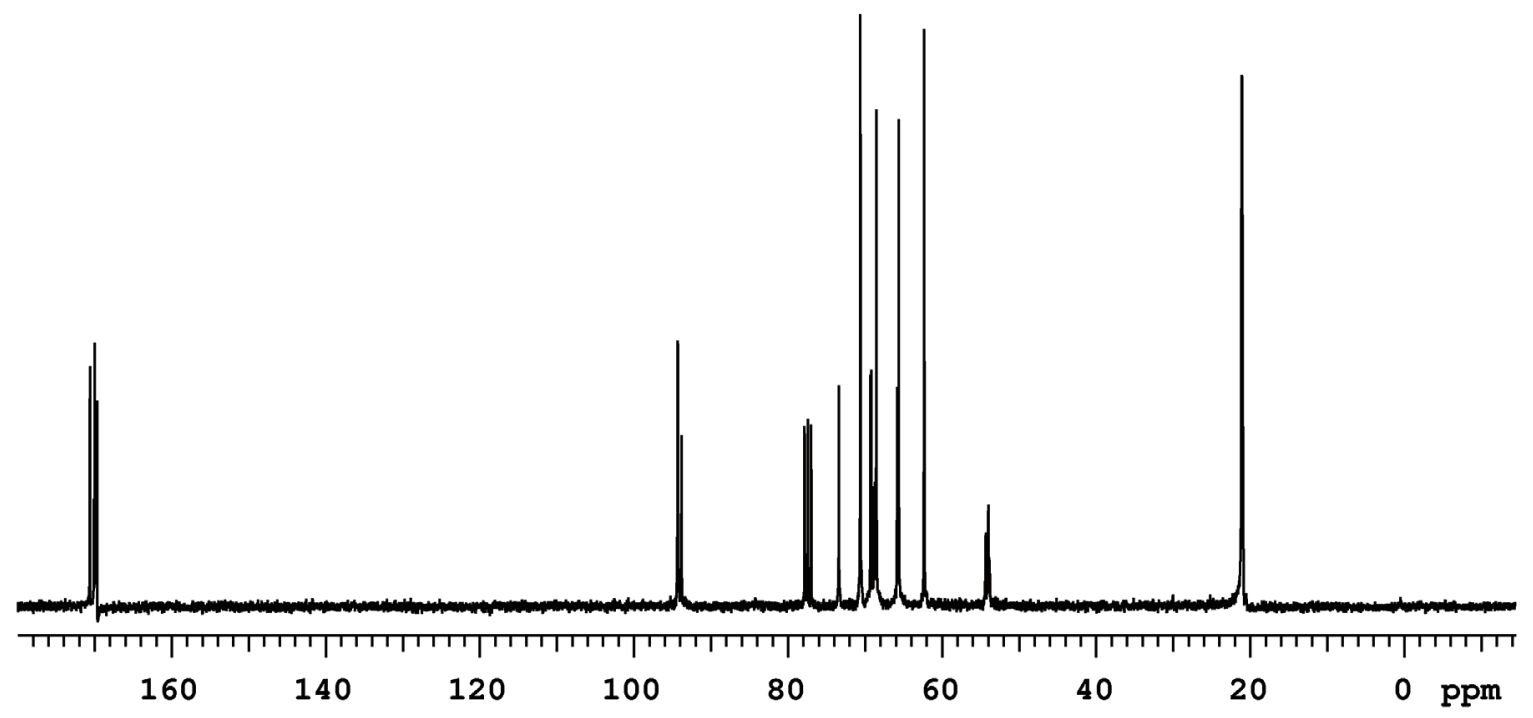

S15 


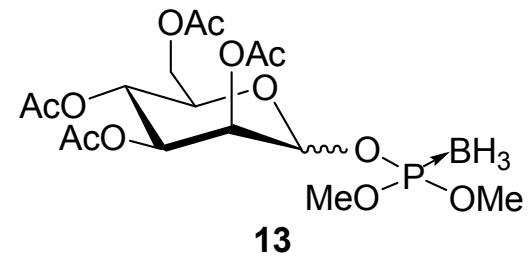

(121.5 MHz, $\mathrm{CDCl}_{3}$ )
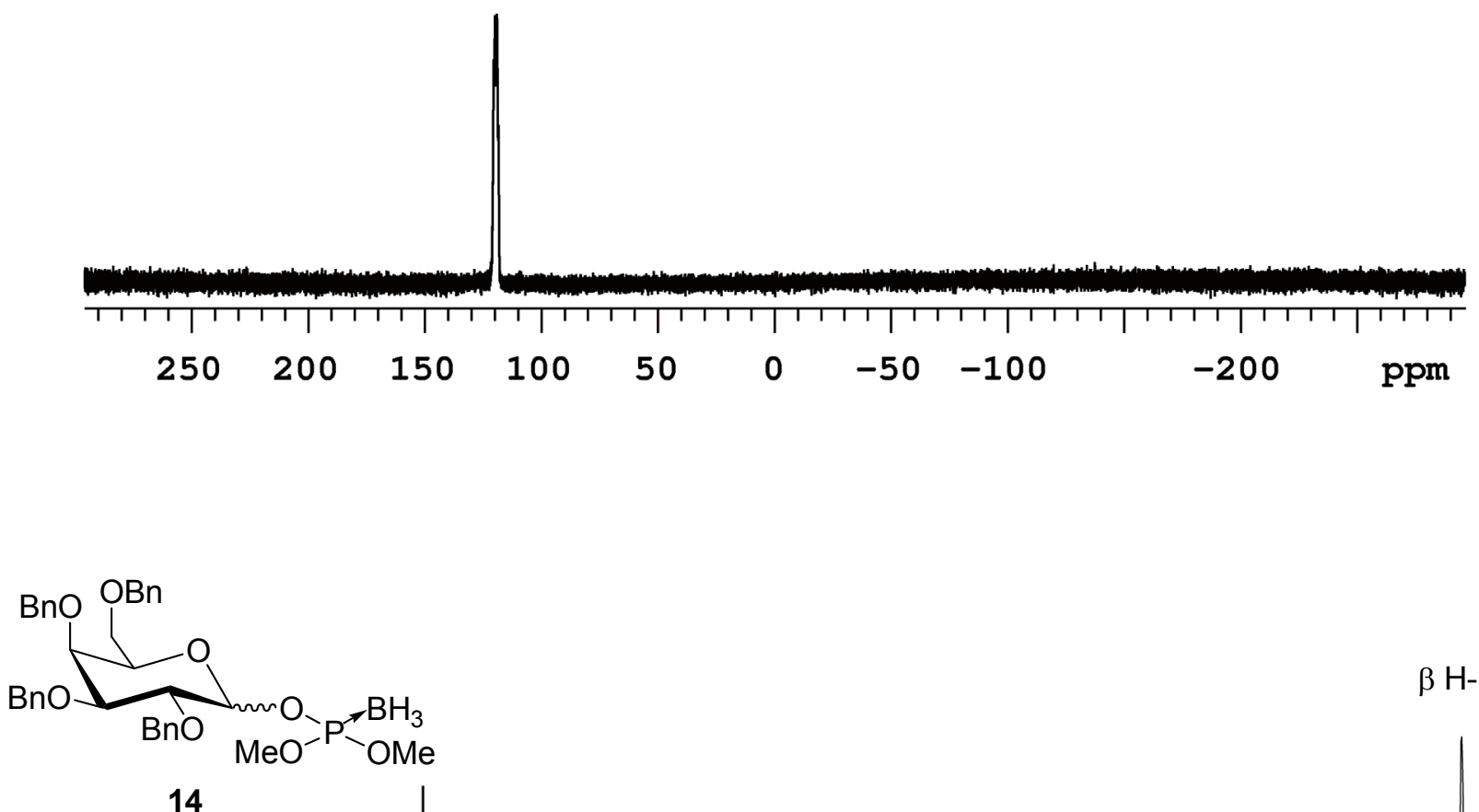

$\beta \mathrm{H}-1$

(300 MHz, $\mathrm{CDCl}_{3}$ )

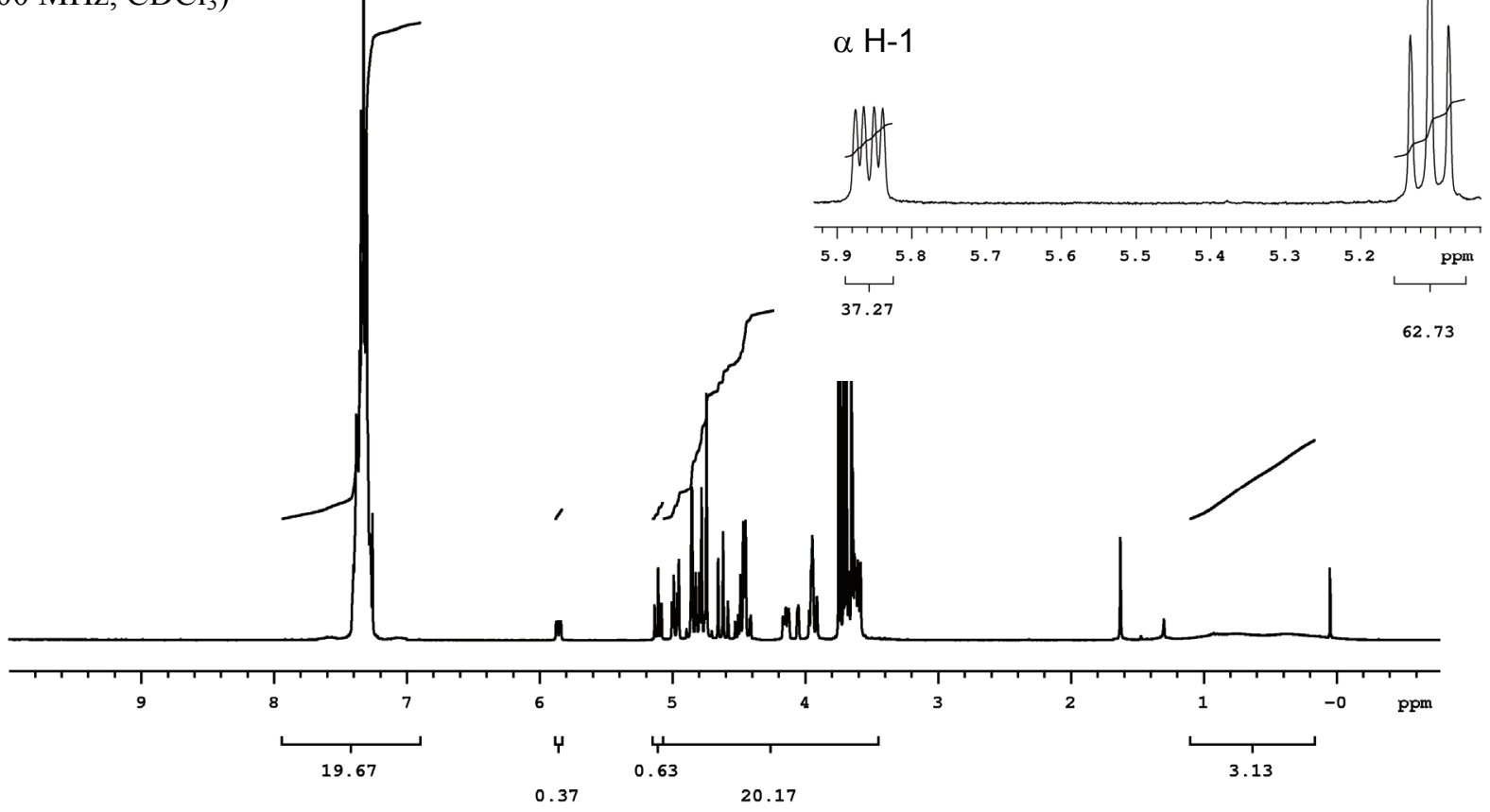



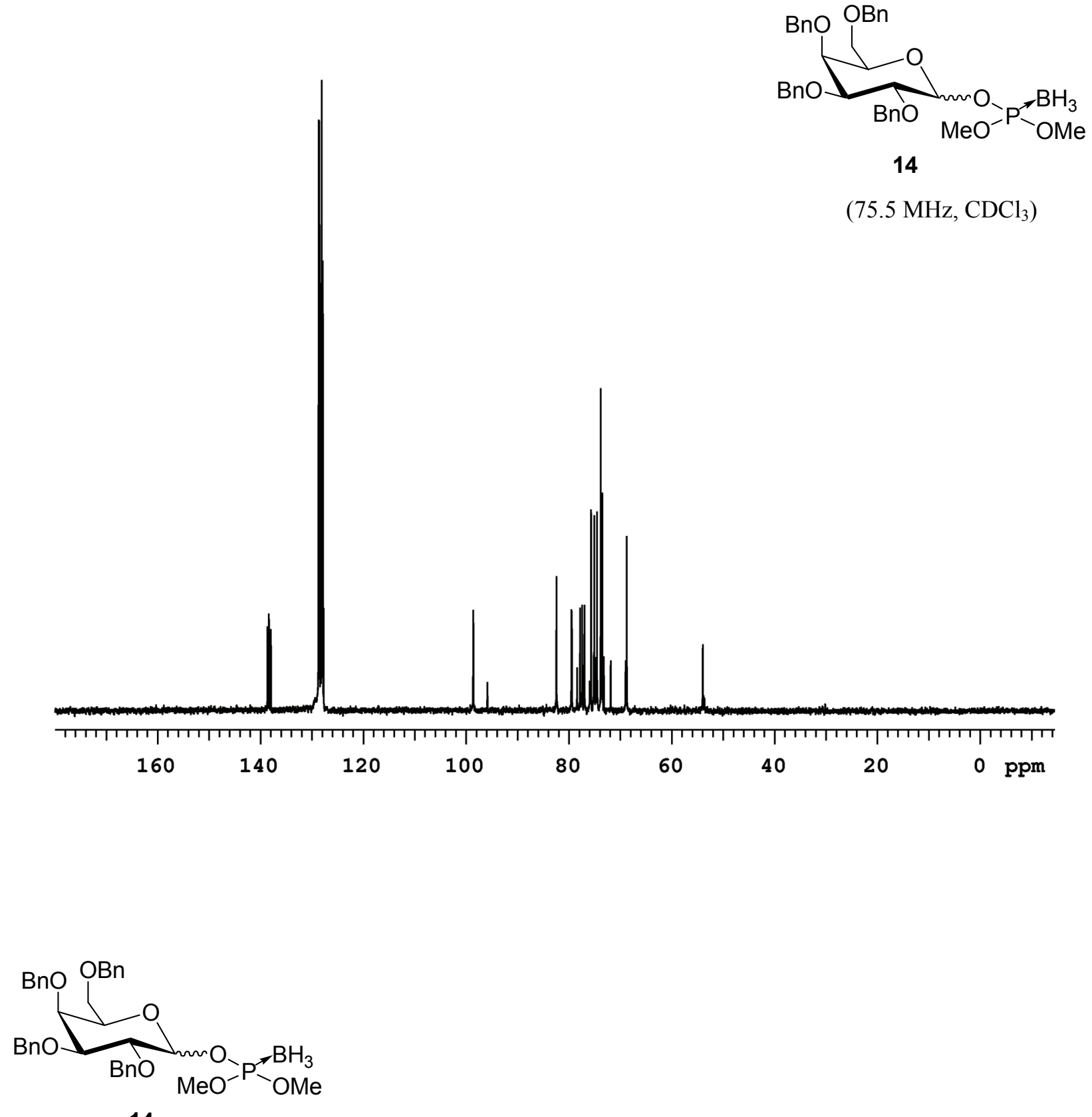

14

(75.5 MHz, $\left.\mathrm{CDCl}_{3}\right)$

(121.5 MHz, $\mathrm{CDCl}_{3}$ )

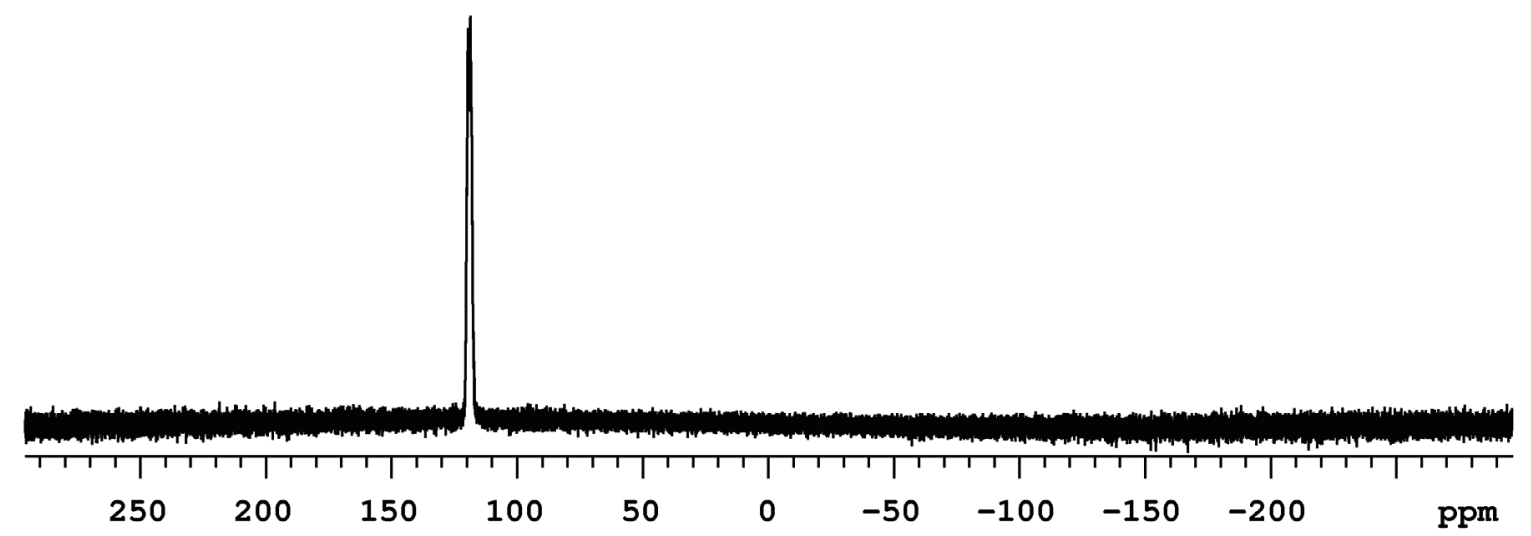




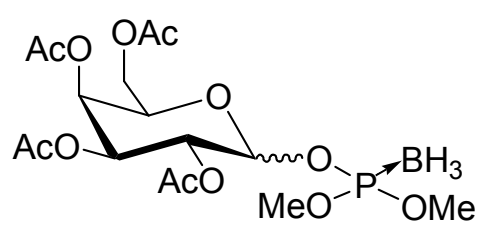

15

(300 MHz, $\mathrm{CDCl}_{3}$ )
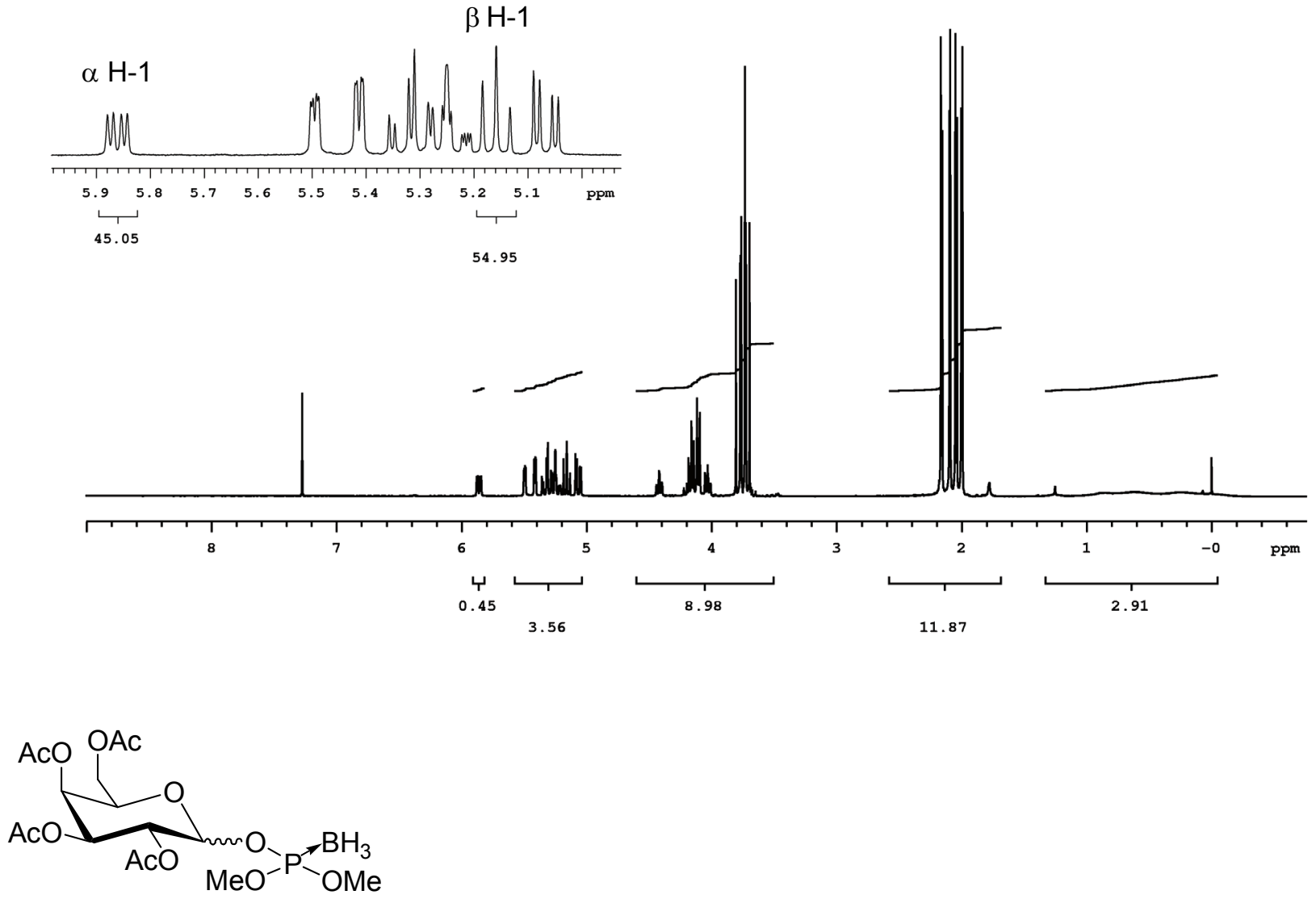

15

(75.5 $\left.\mathrm{MHz}, \mathrm{CDCl}_{3}\right)$

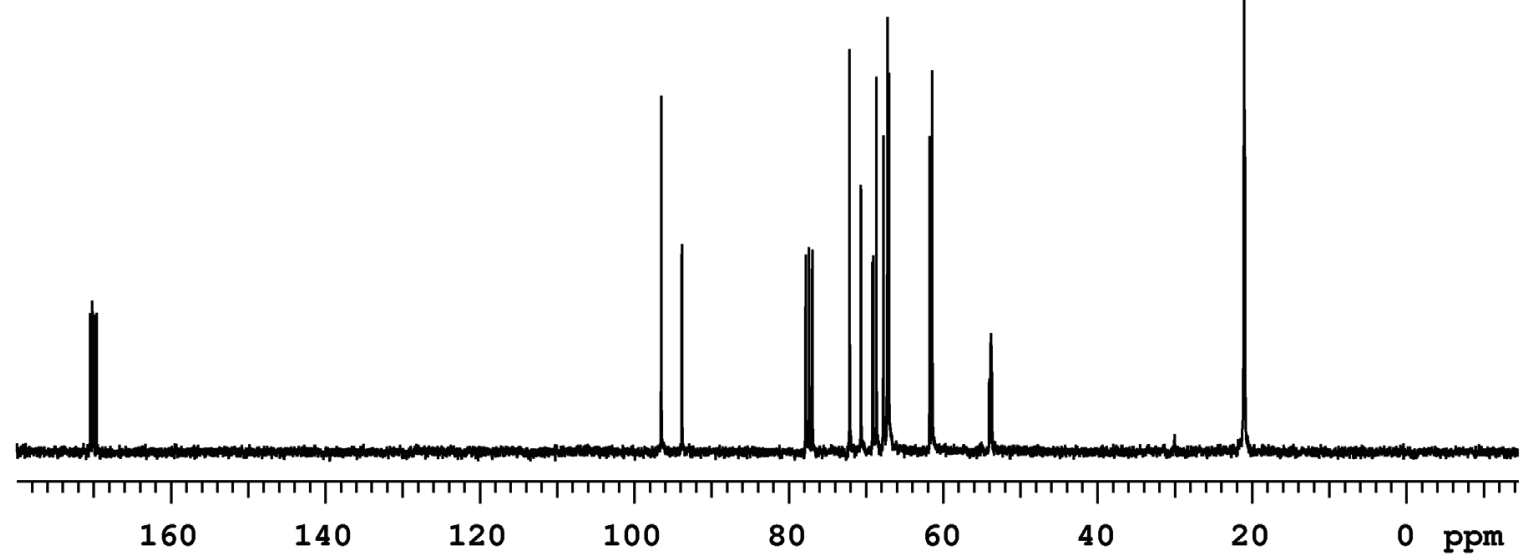




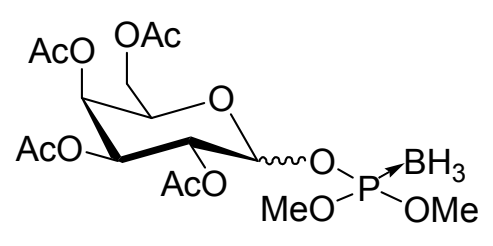

15

(121.5 MHz, $\mathrm{CDCl}_{3}$ )
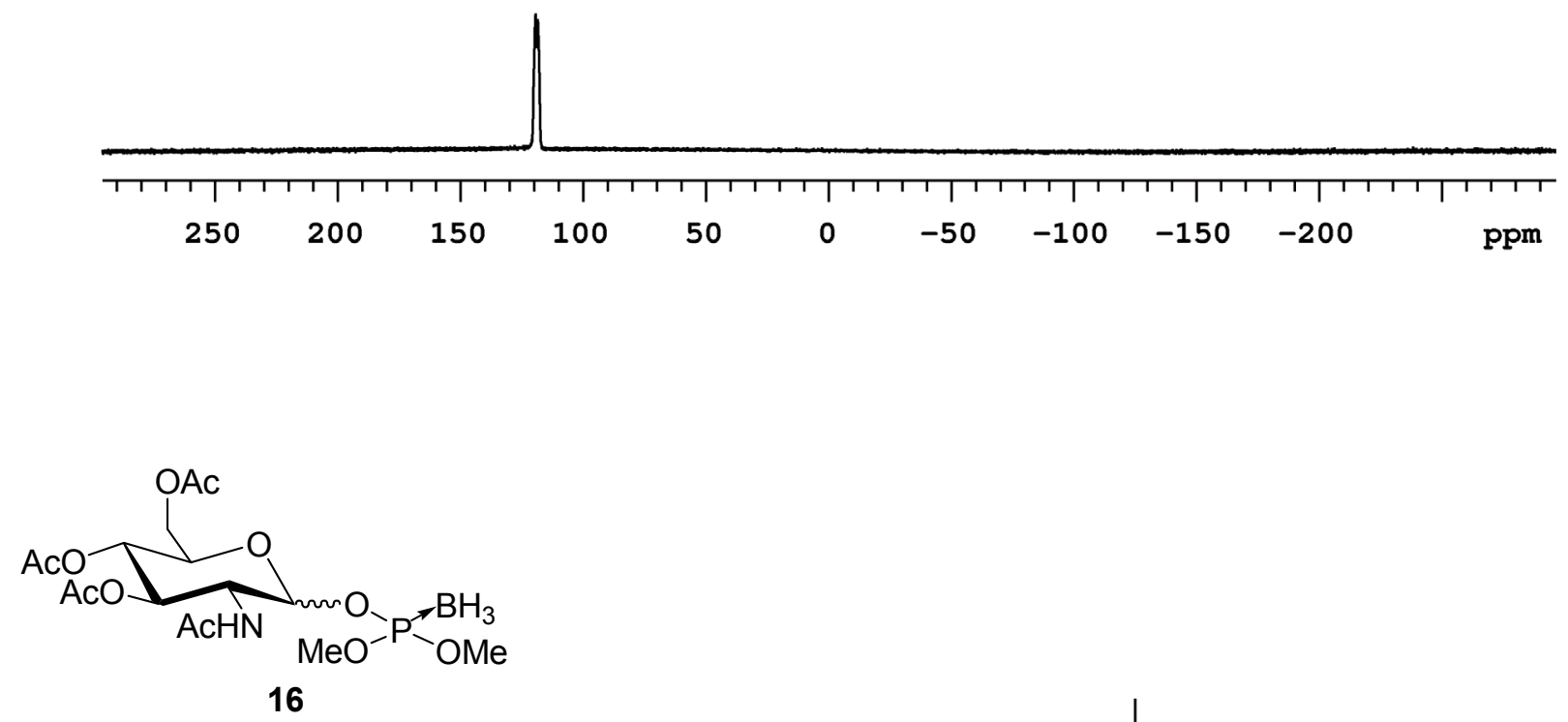

(300 MHz, $\mathrm{CDCl}_{3}$ )

$\alpha \mathrm{NH}, \beta \mathrm{NH}$ $\alpha \mathrm{H}-1$
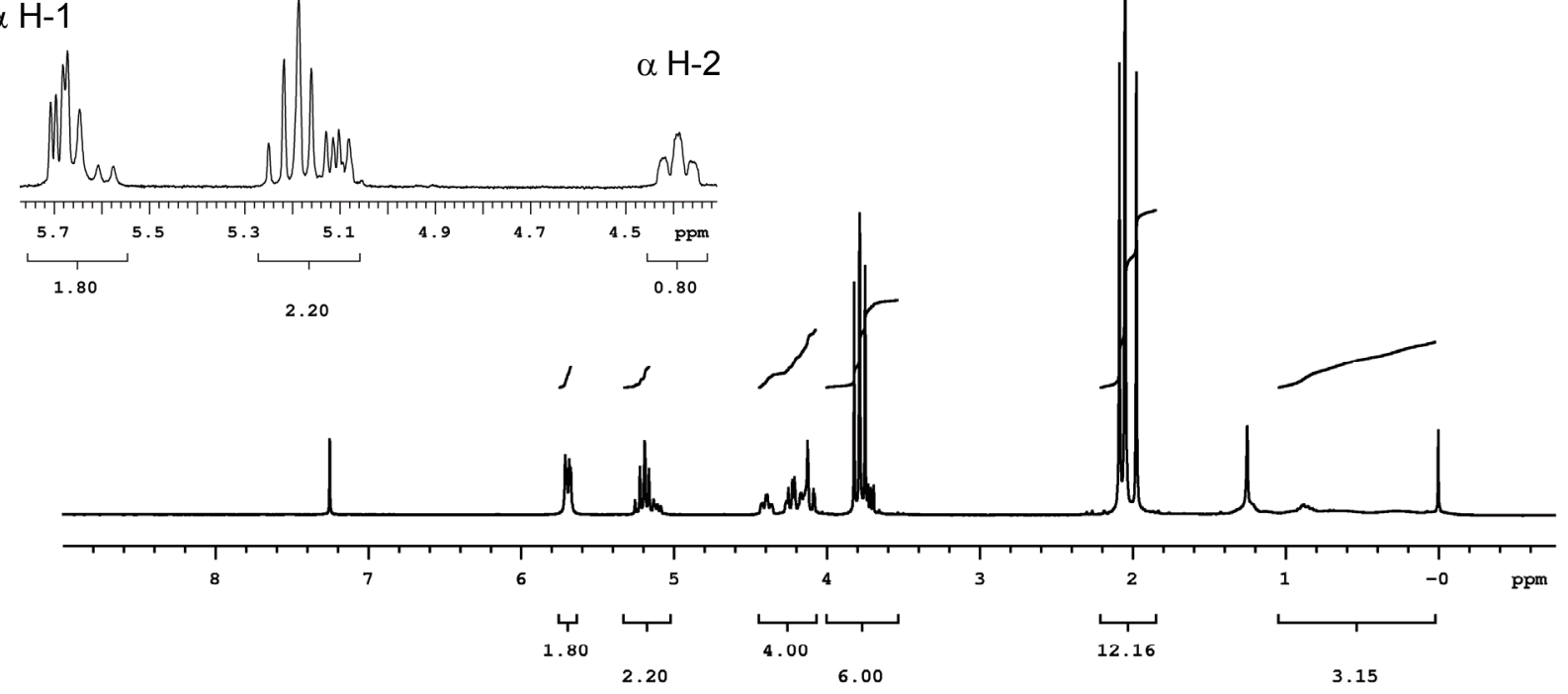


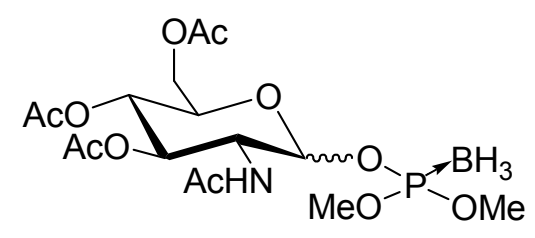

16

(75.5 MHz, $\mathrm{CDCl}_{3}$ )
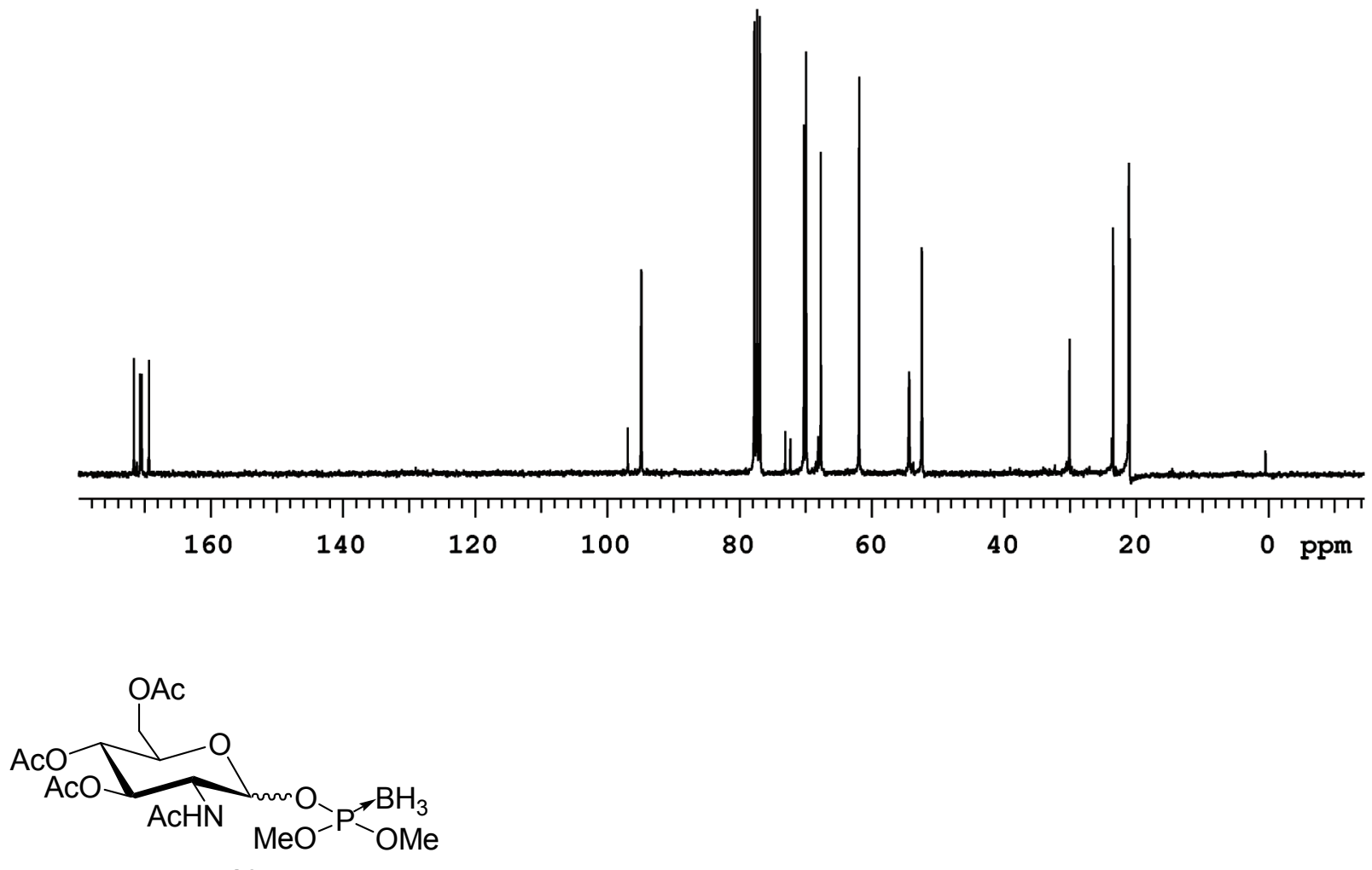

16

(121.5 MHz, $\mathrm{CDCl}_{3}$ )

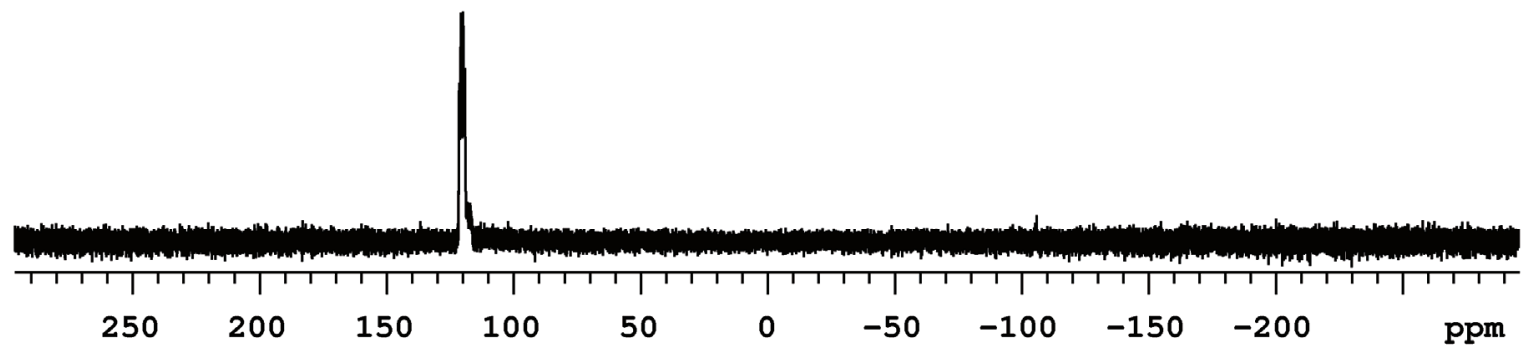



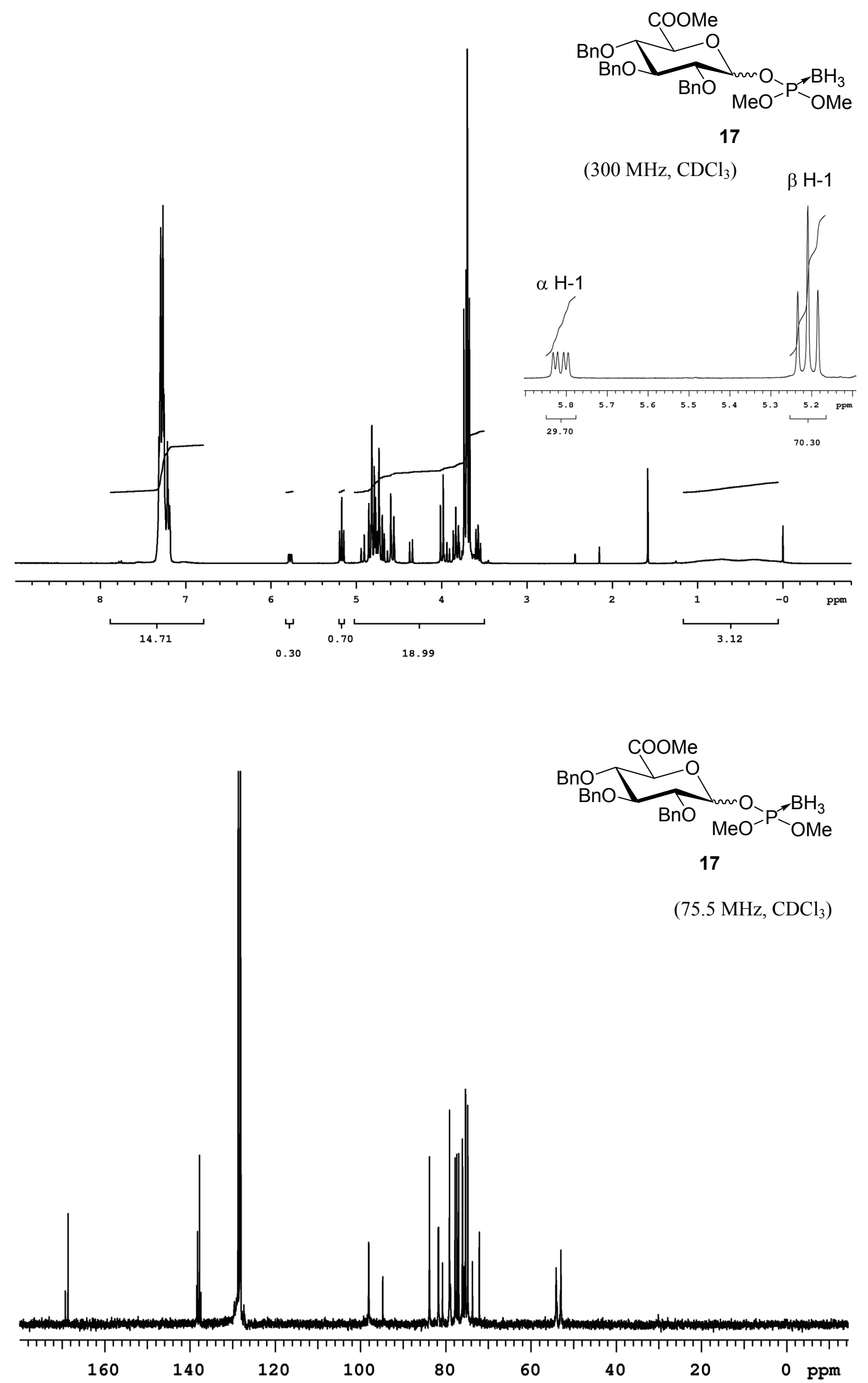


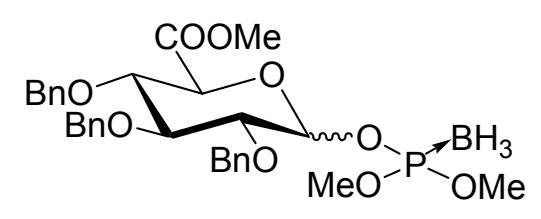

17

(121.5 MHz, $\mathrm{CDCl}_{3}$ )
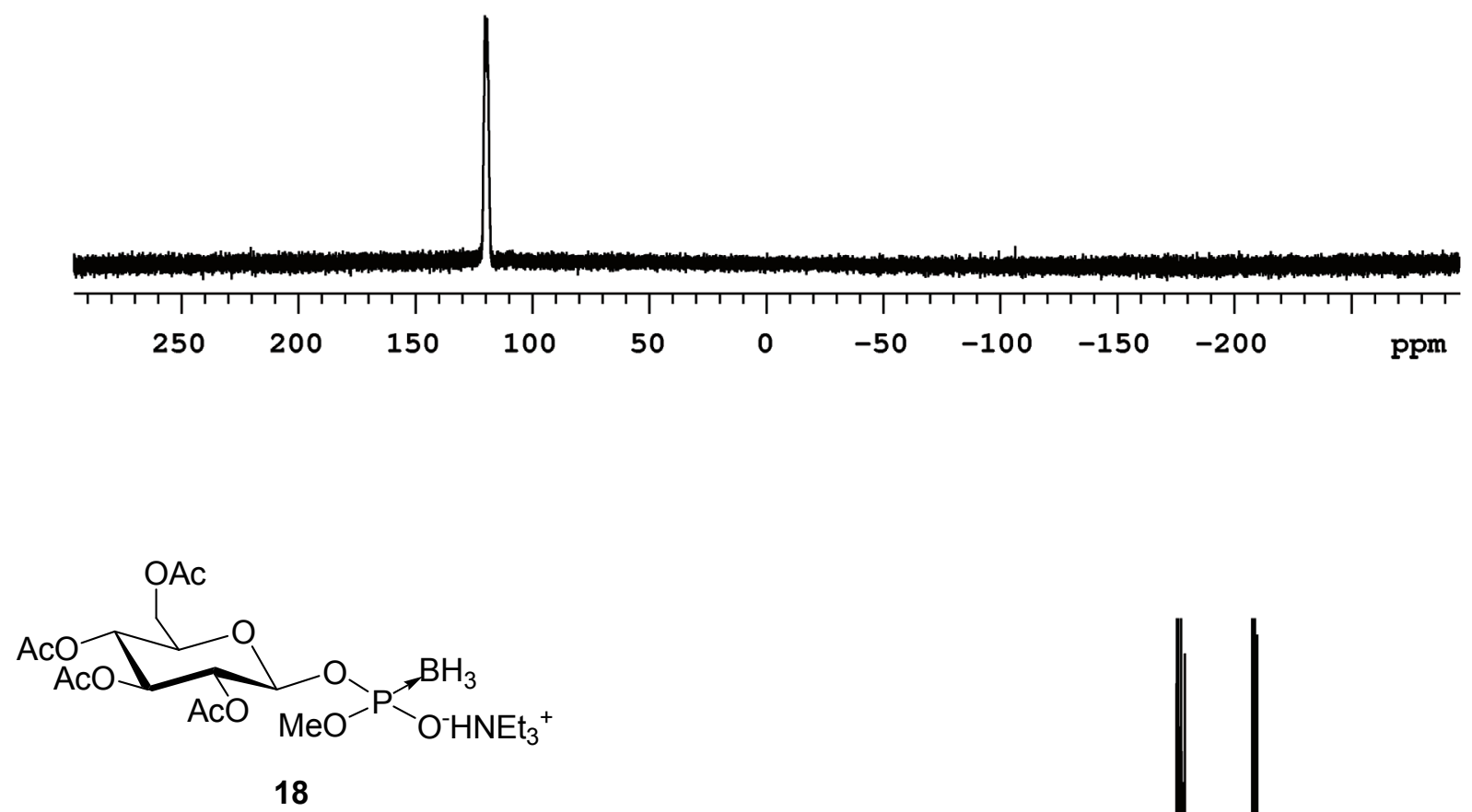

(300 MHz, $\mathrm{CDCl}_{3}$ )

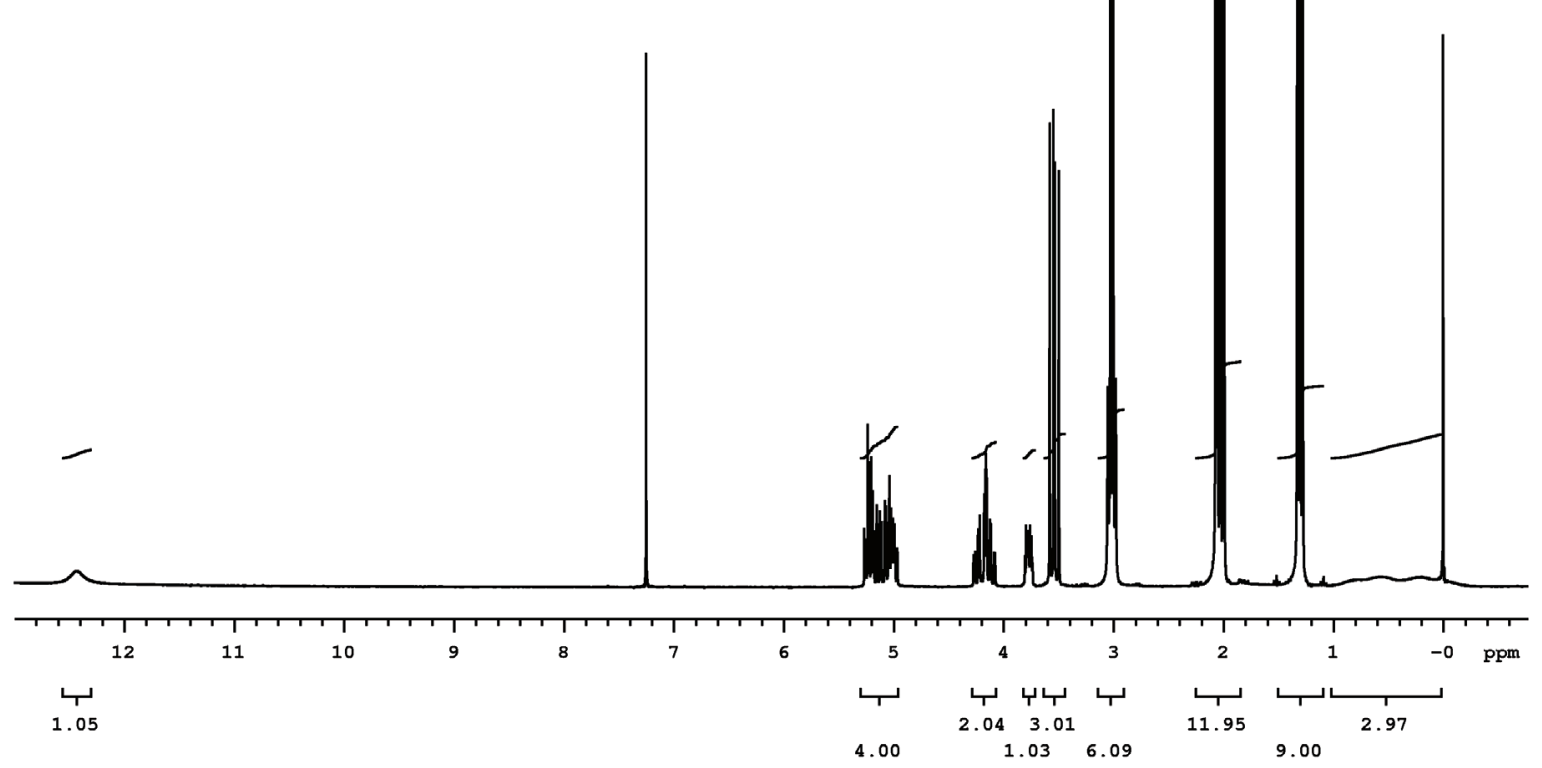




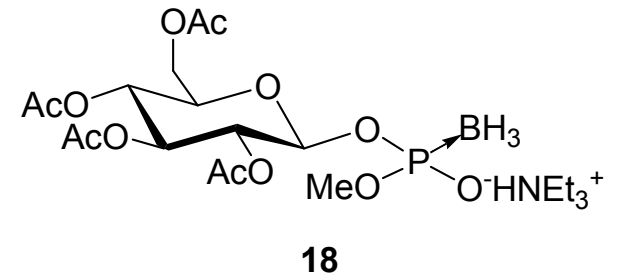

(75.5 MHz, $\mathrm{CDCl}_{3}$ )
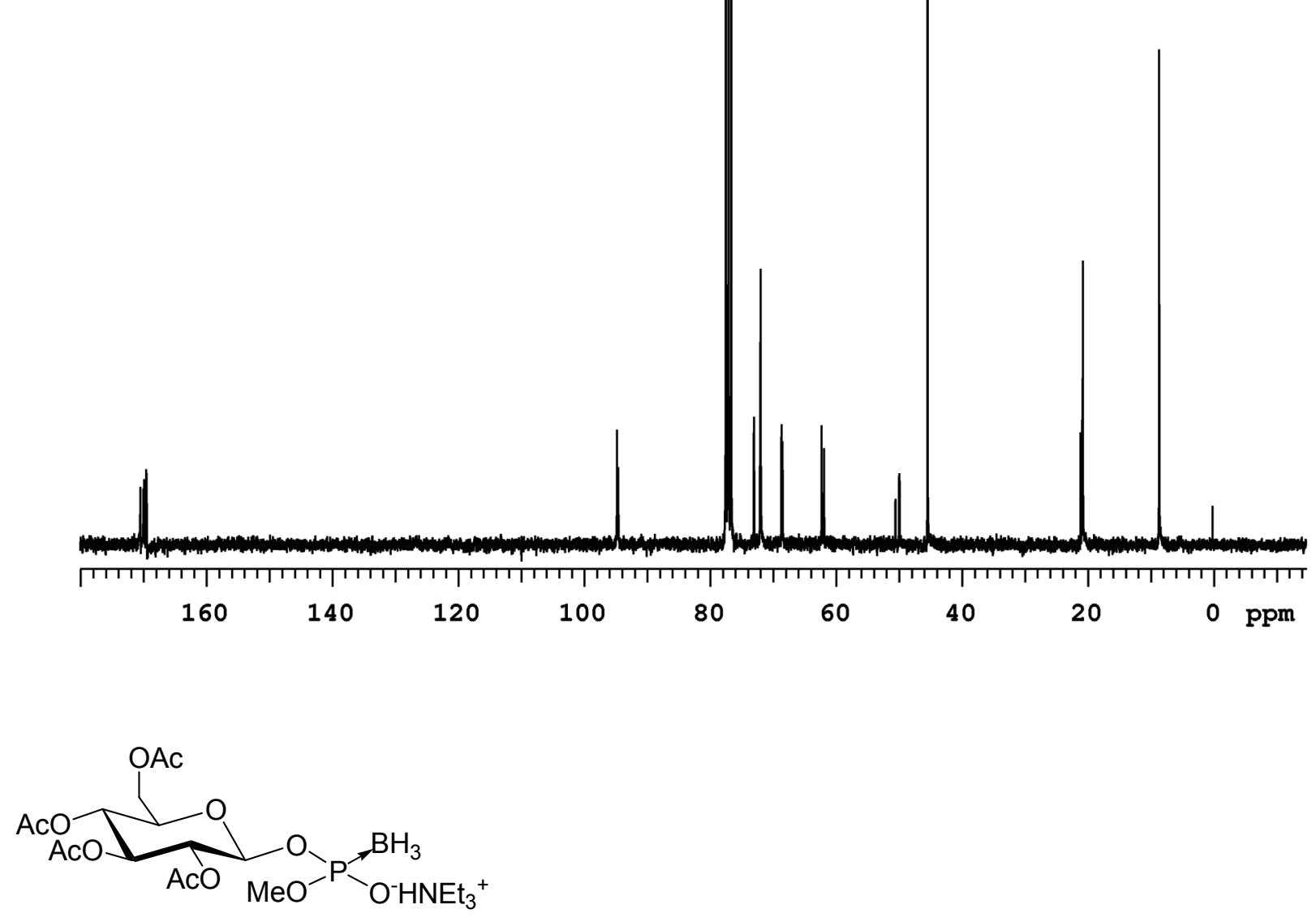

18

(121.5 MHz, $\mathrm{CDCl}_{3}$ )

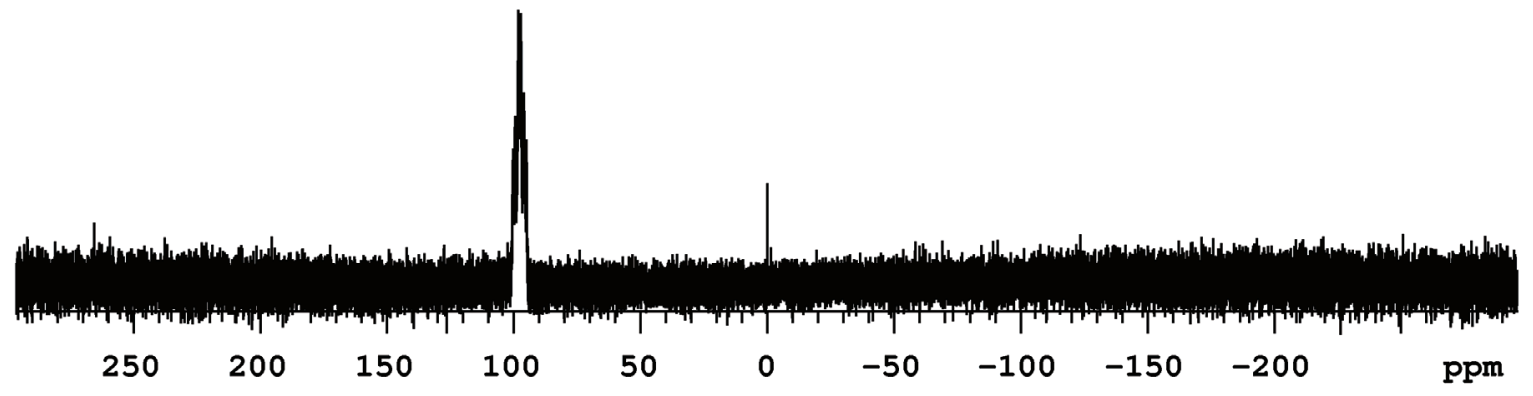




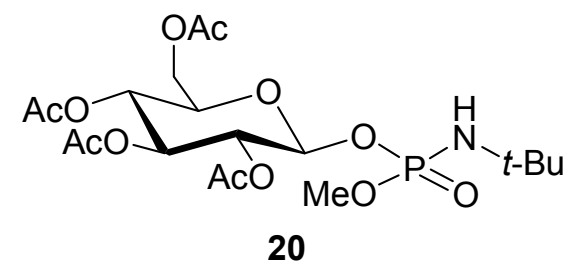

(300 MHz, $\mathrm{CDCl}_{3}$ )

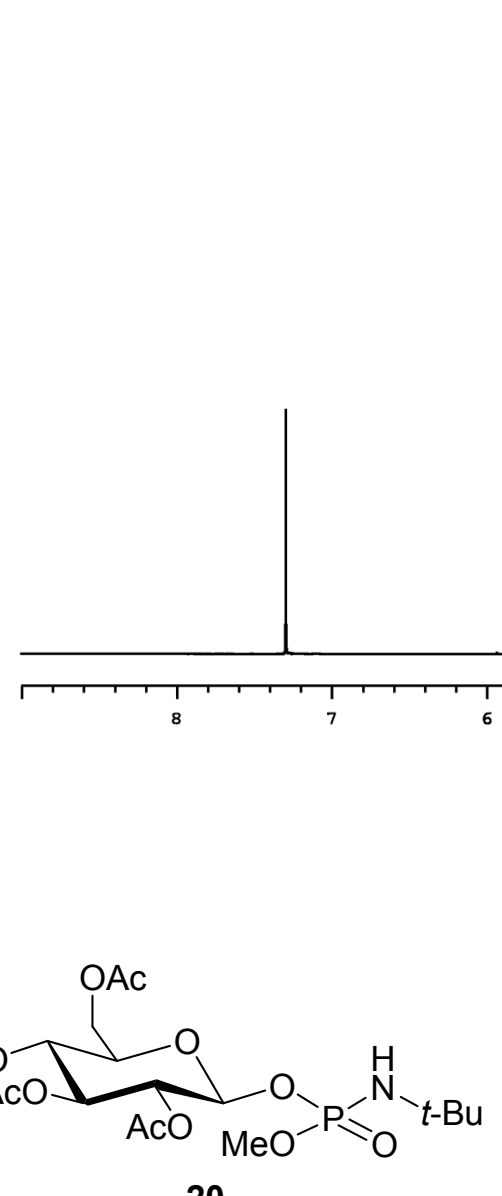

20

(75.5 MHz, $\mathrm{CDCl}_{3}$ )

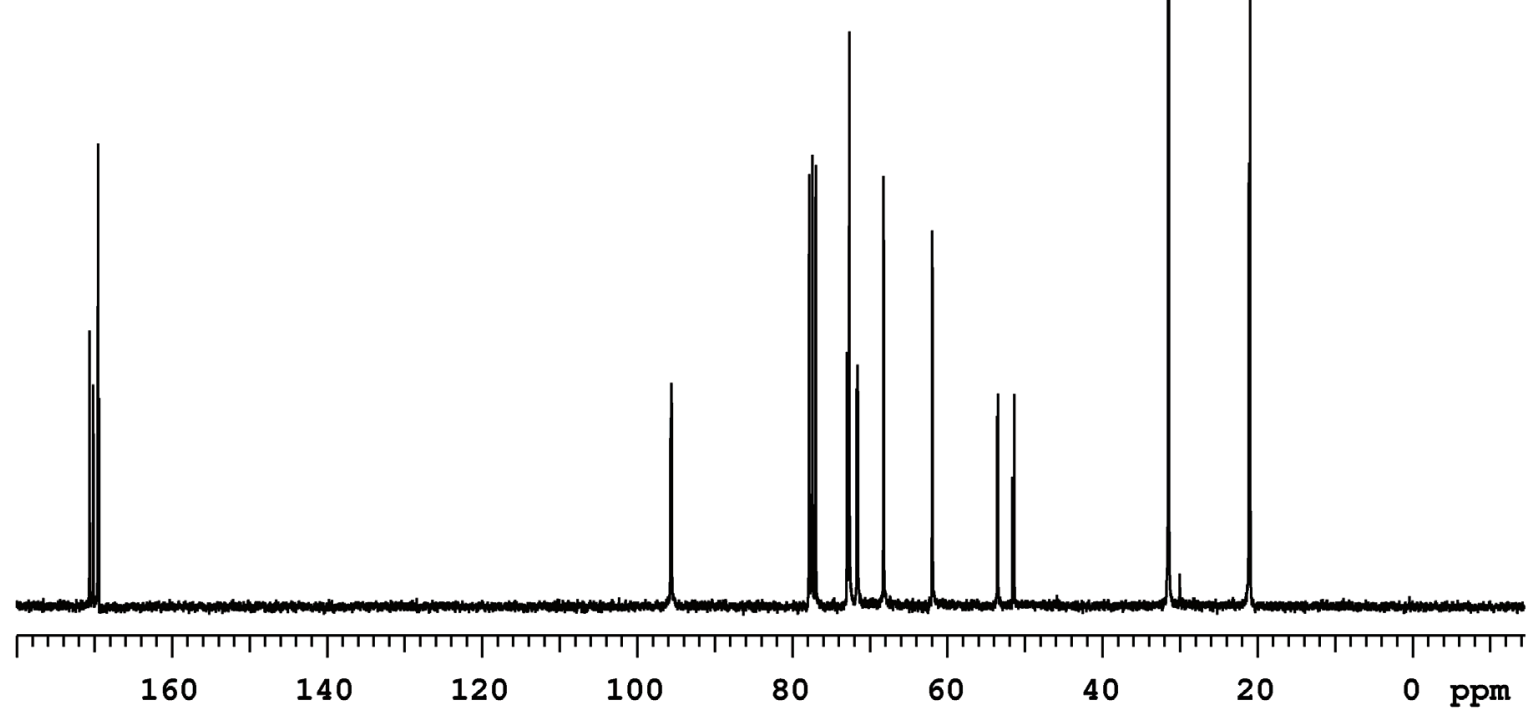




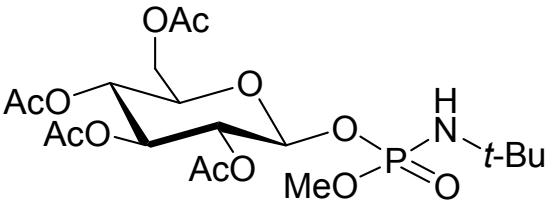

20

(121.5 MHz, $\mathrm{CDCl}_{3}$ )
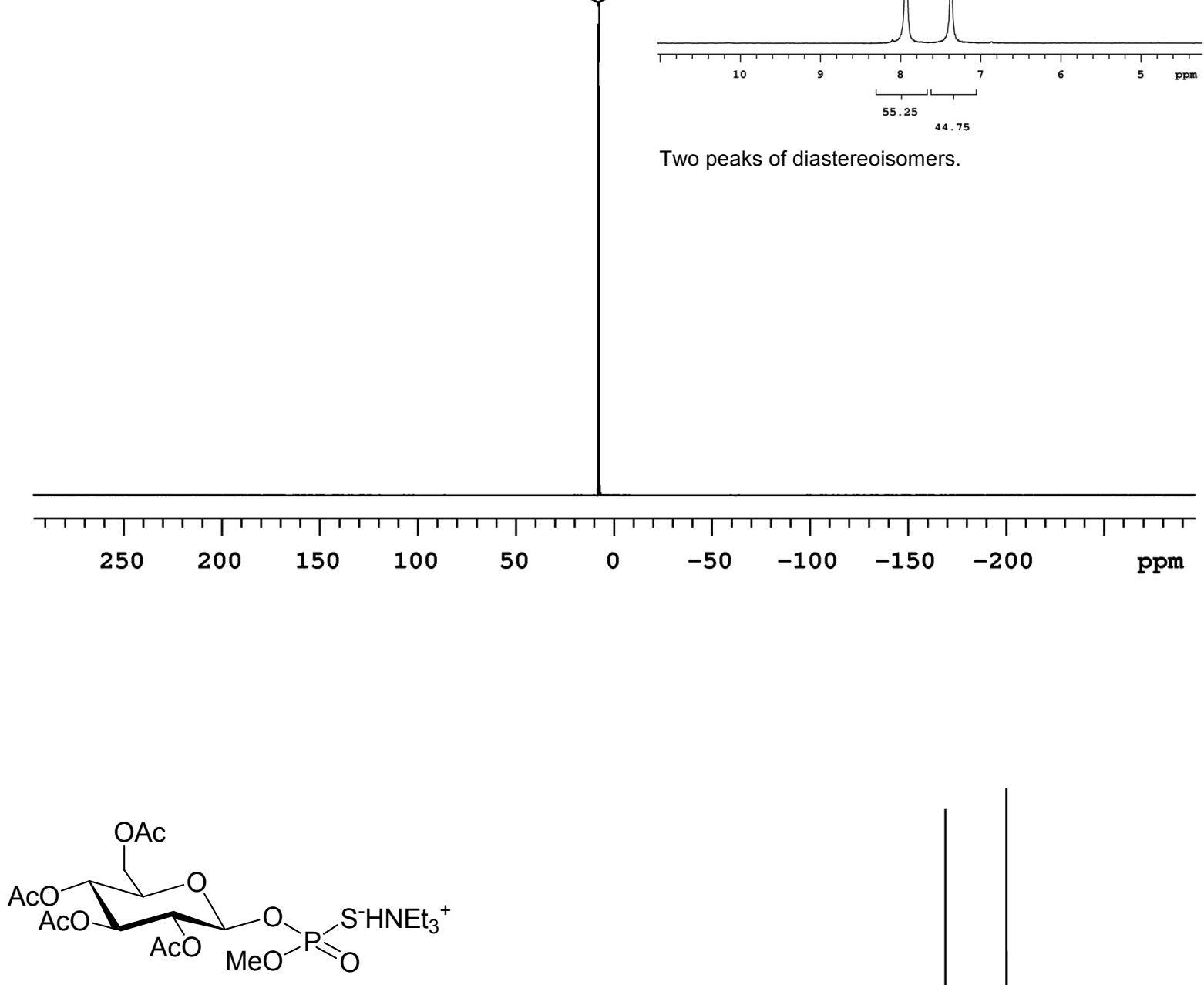

21

$\left(300 \mathrm{MHz}, \mathrm{CDCl}_{3}\right)$

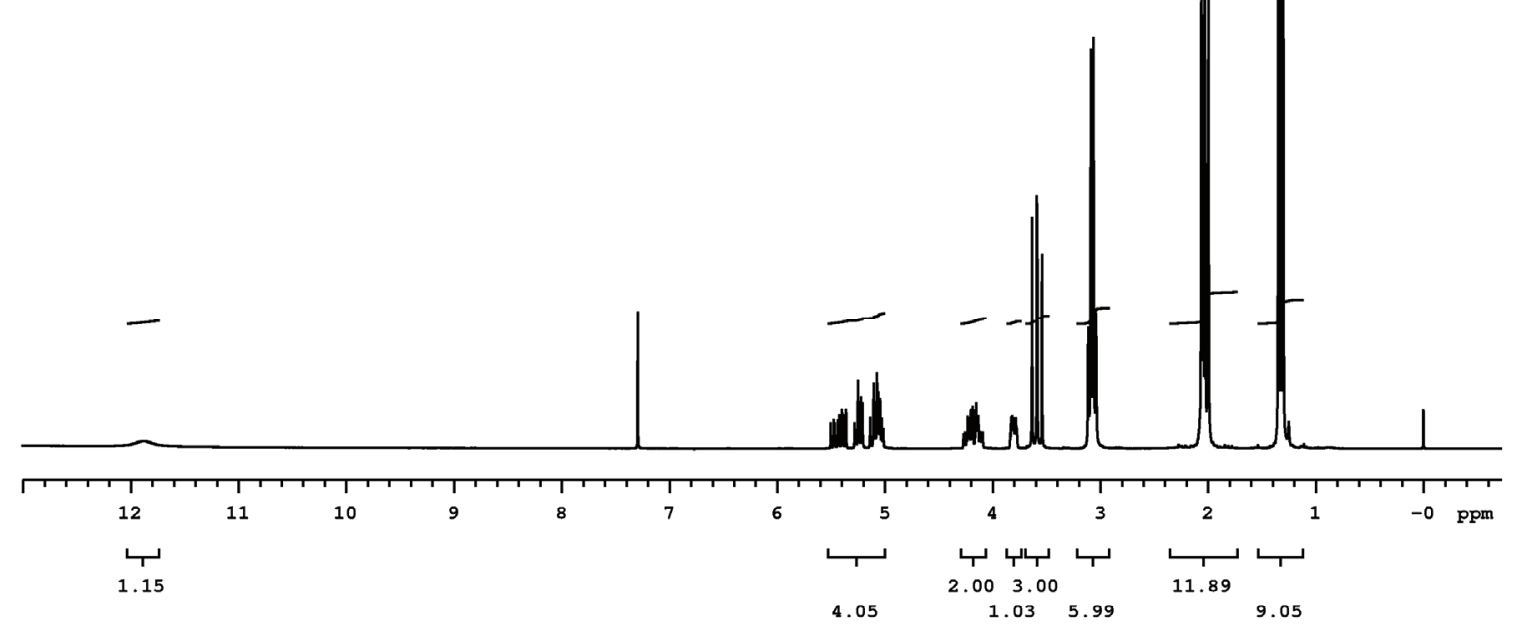




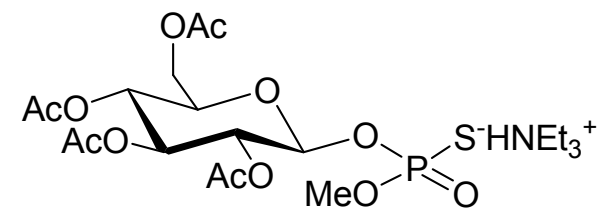

21

(75.5 MHz, $\mathrm{CDCl}_{3}$ )

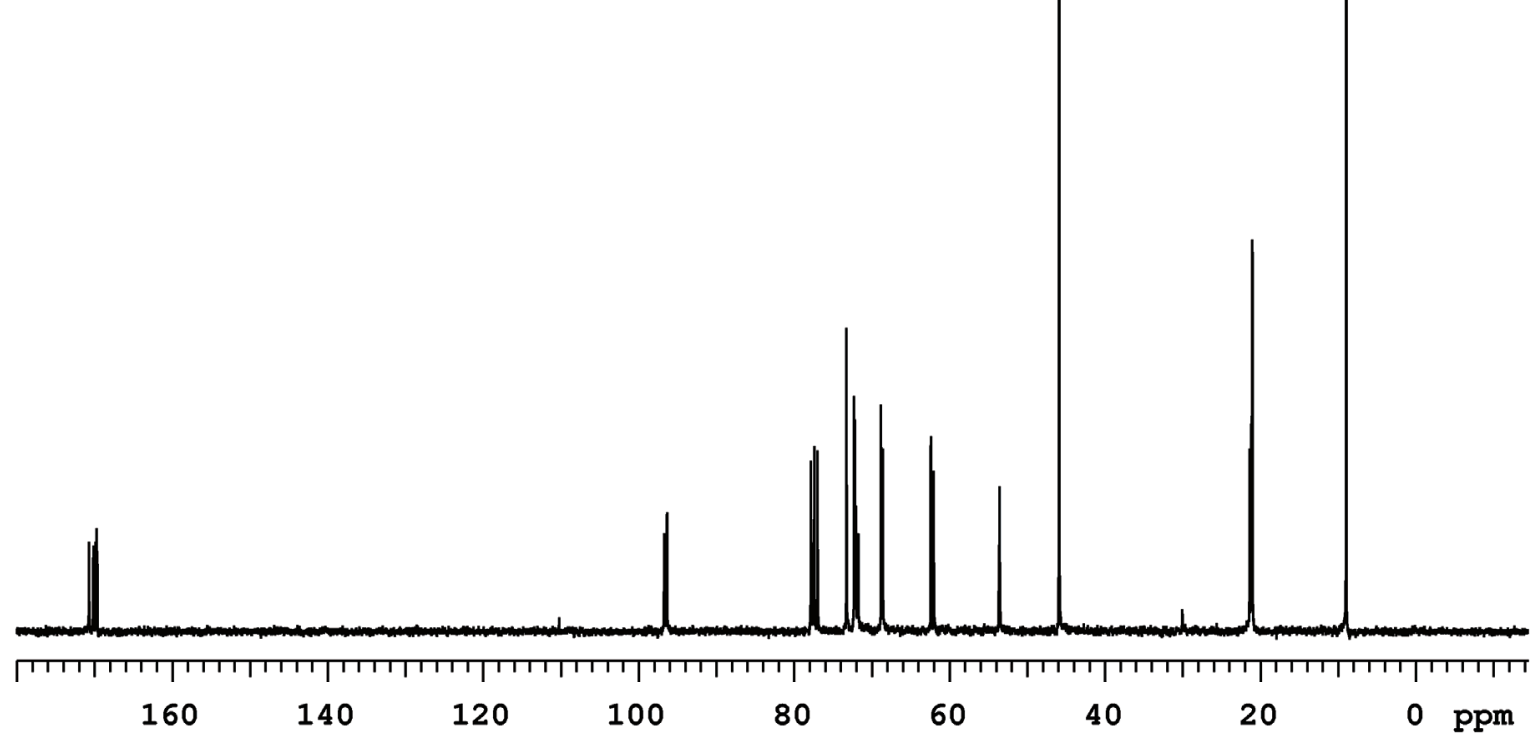

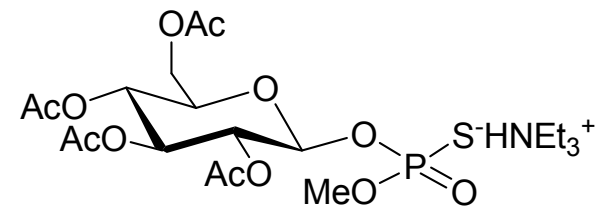

21

(121.5 $\mathrm{MHz}, \mathrm{CDCl}_{3}$ )

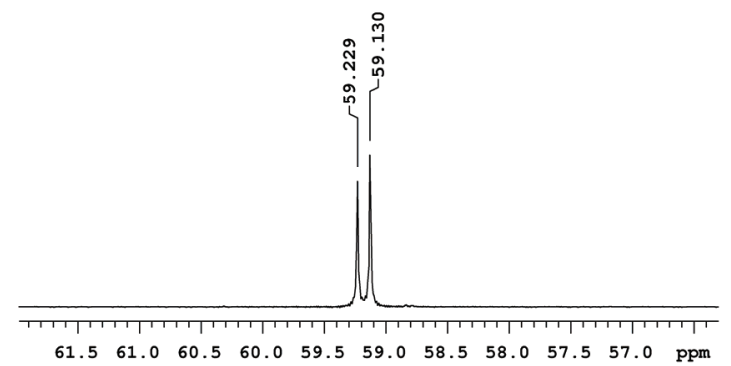

Two peaks of diastereoisomers. 


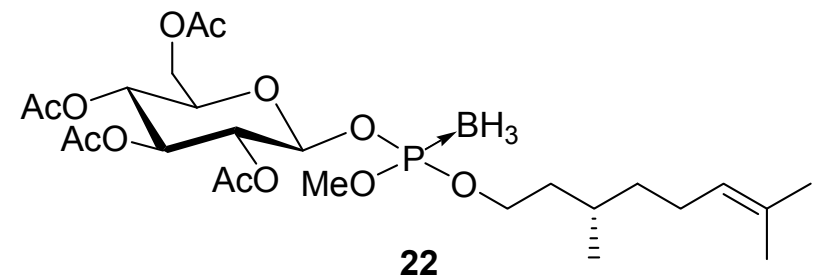

(300 MHz, $\mathrm{CDCl}_{3}$ )
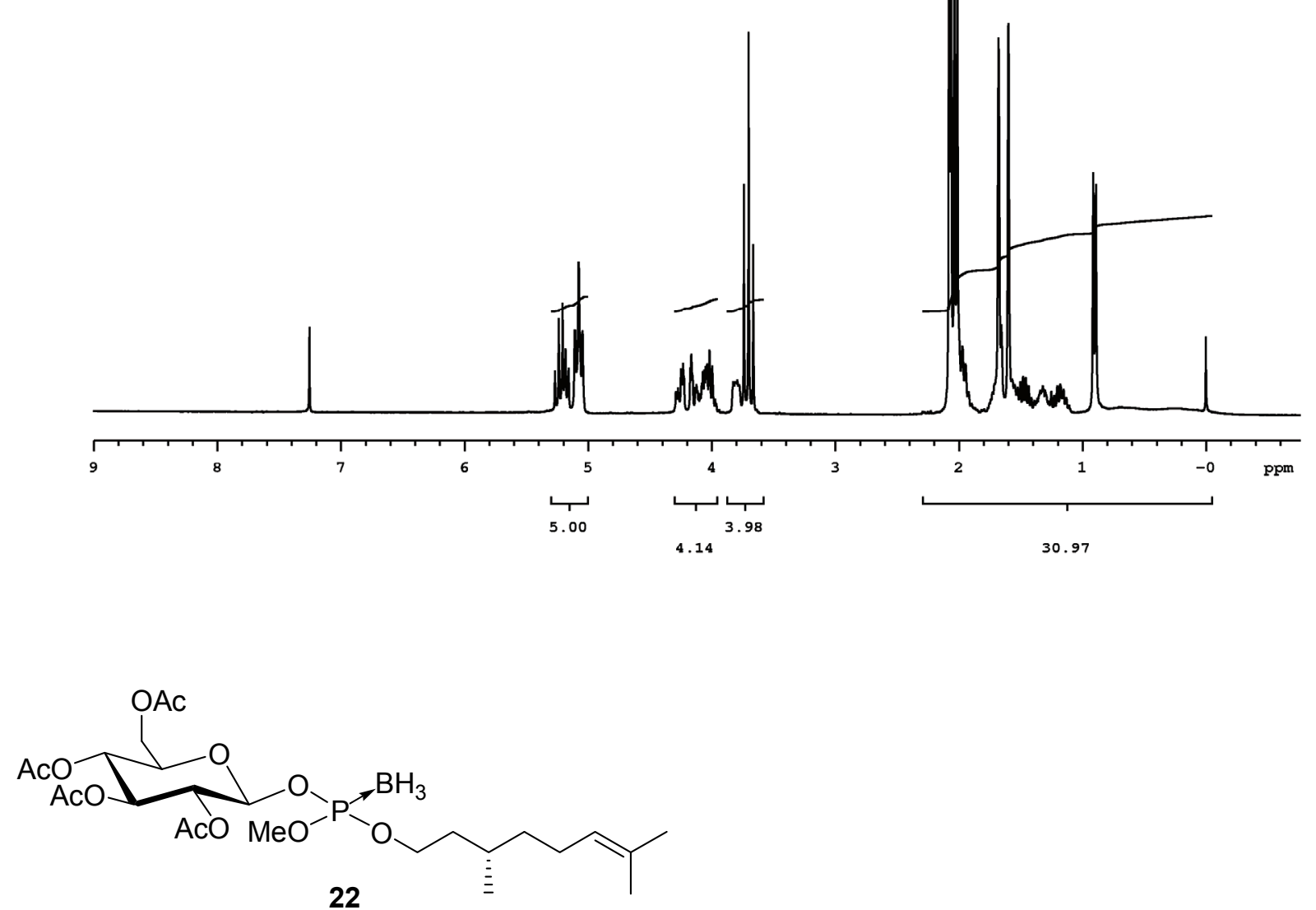

(75.5 $\mathrm{MHz}, \mathrm{CDCl}_{3}$ )

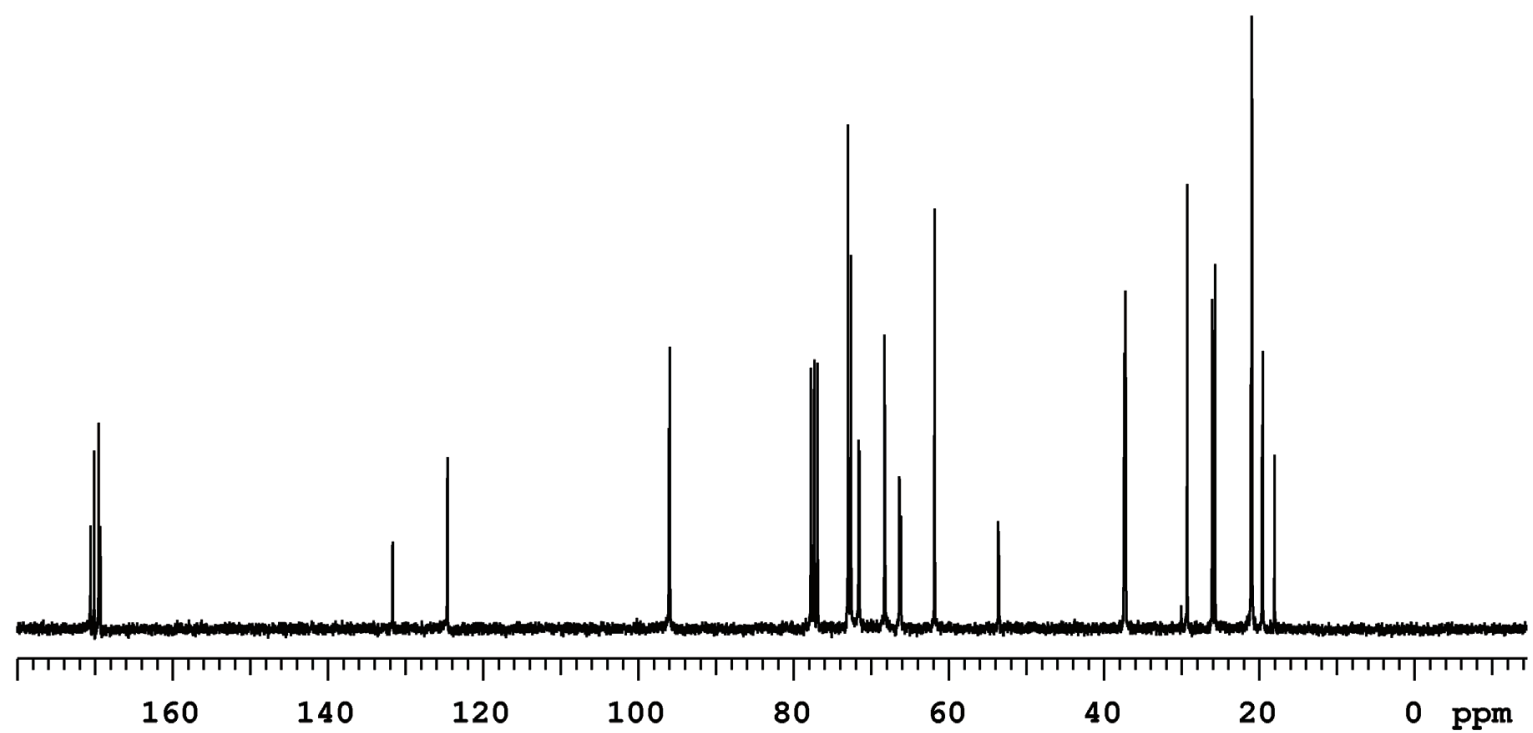




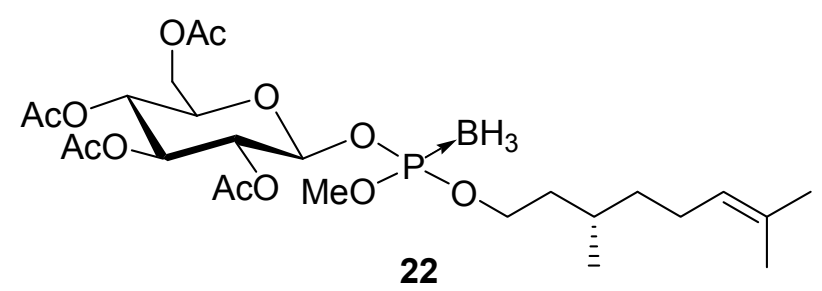

(121.5 MHz, $\mathrm{CDCl}_{3}$ )
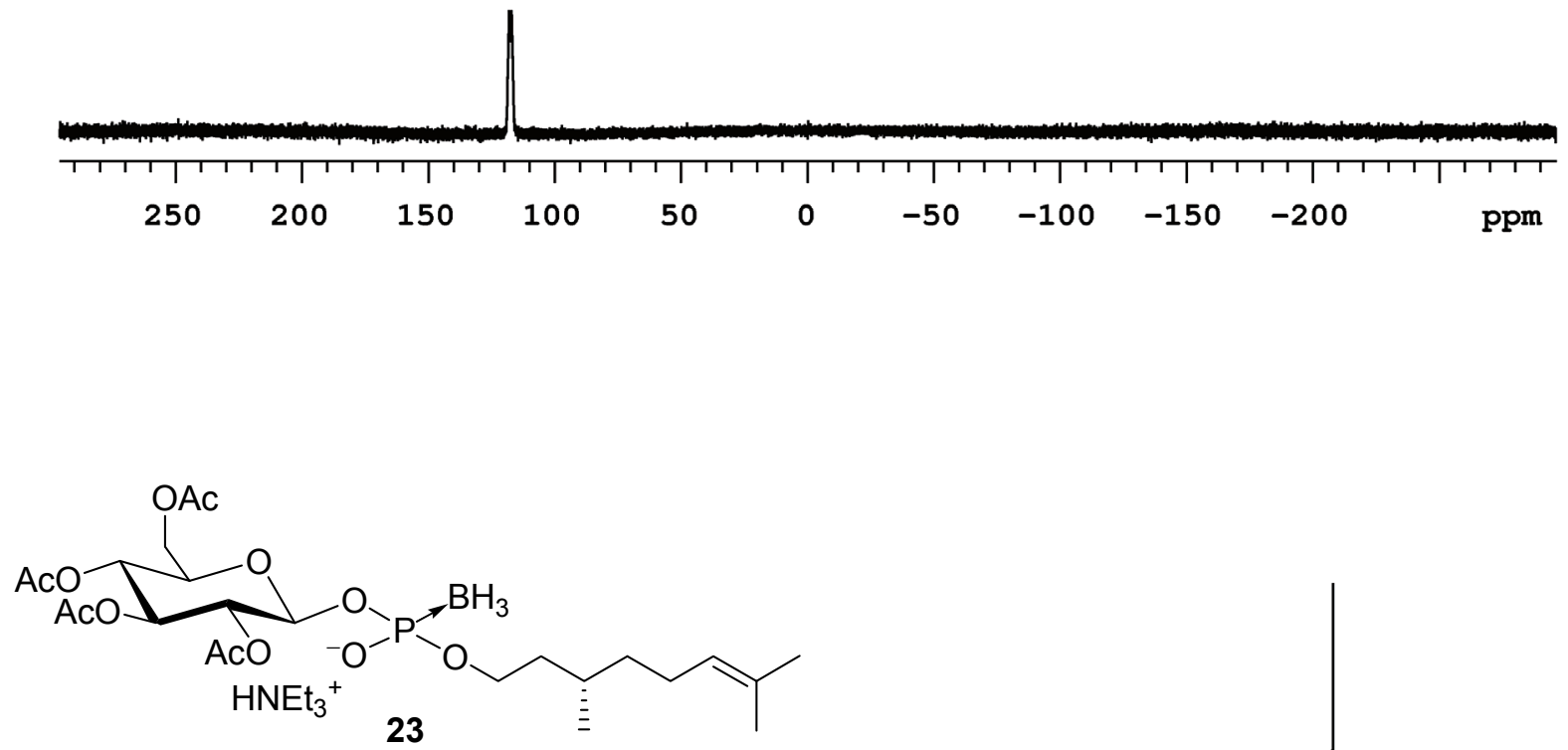

(300 MHz, $\mathrm{CDCl}_{3}$ )

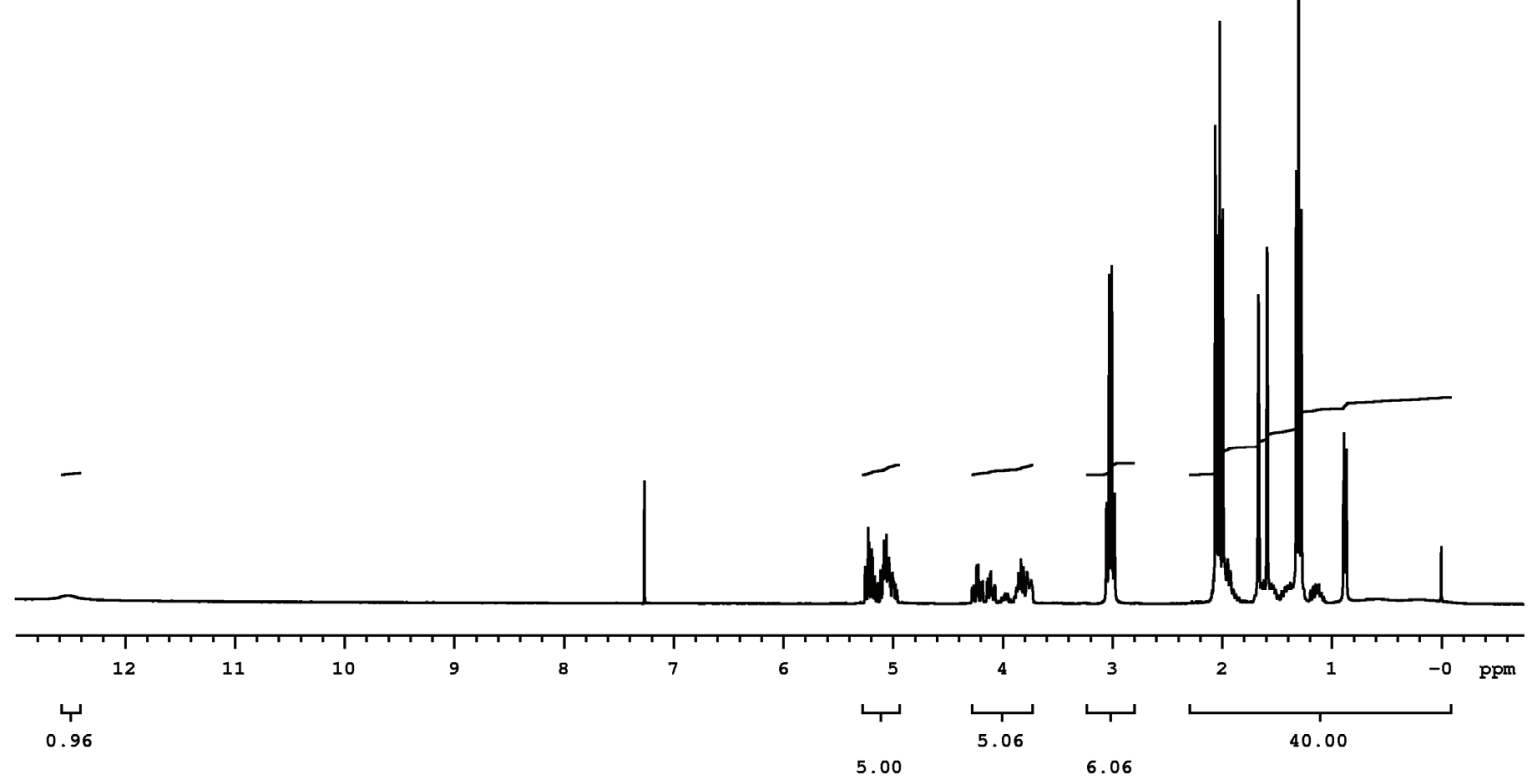




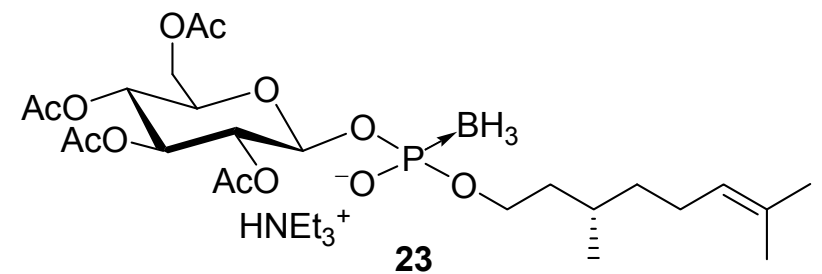

(75.5 MHz, $\mathrm{CDCl}_{3}$ )
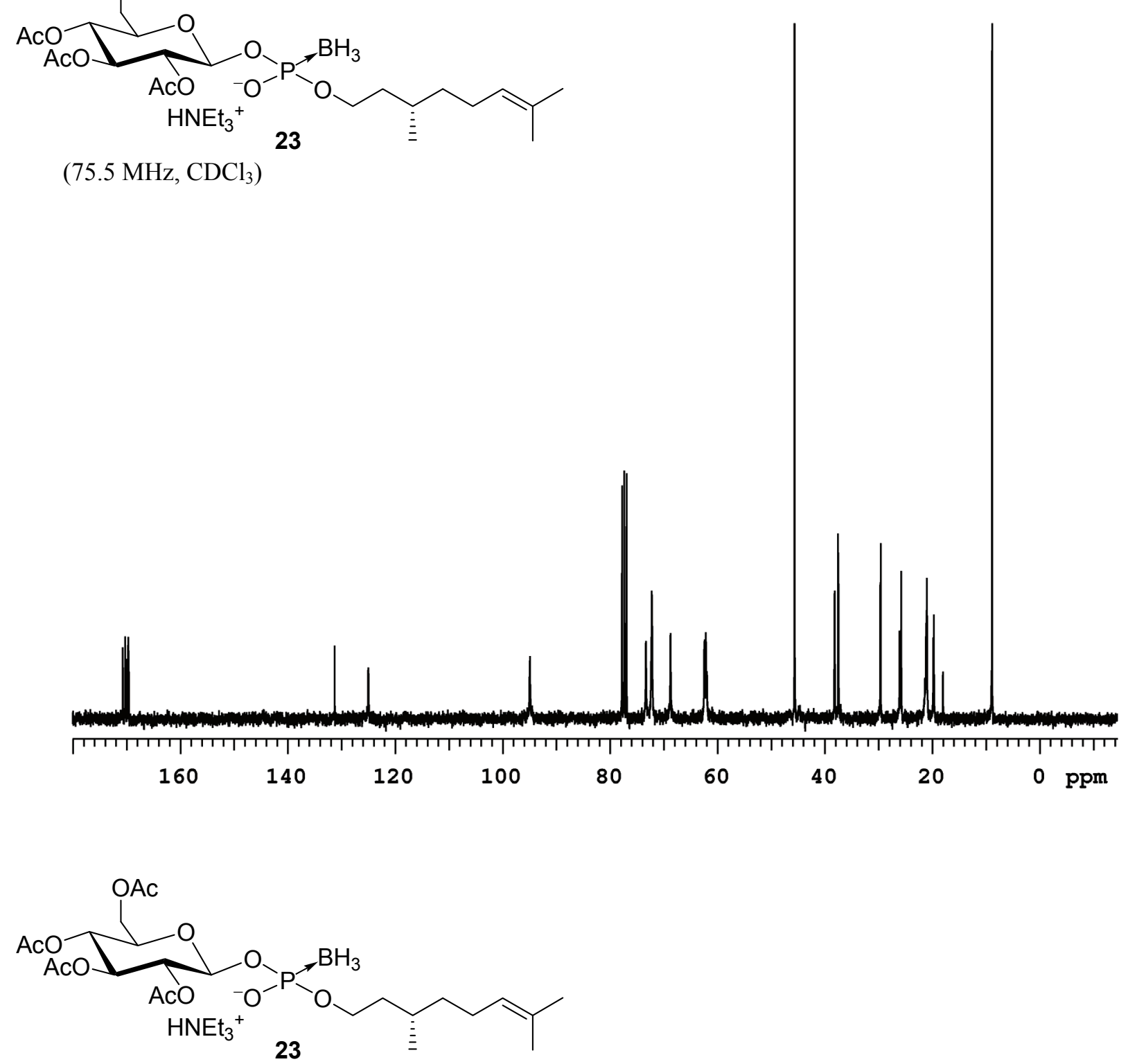

(121.5 MHz, $\mathrm{CDCl}_{3}$ )

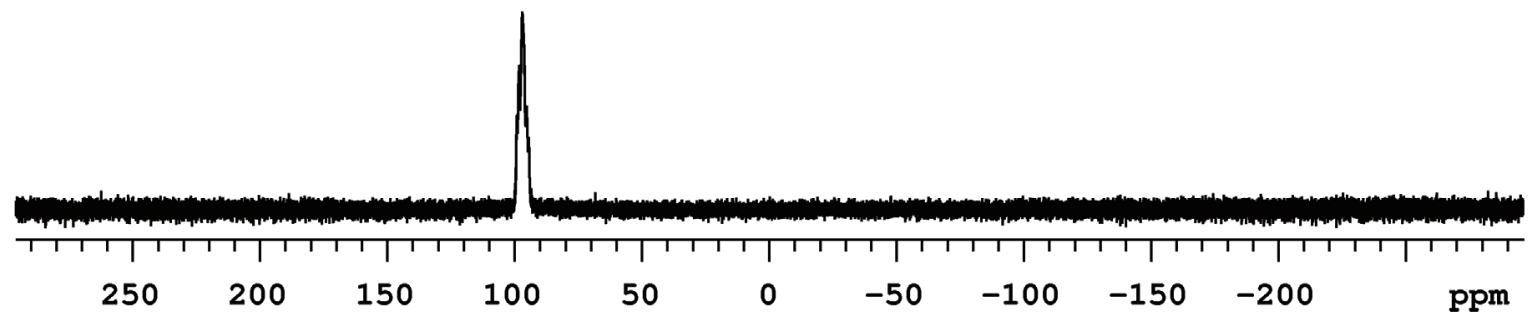




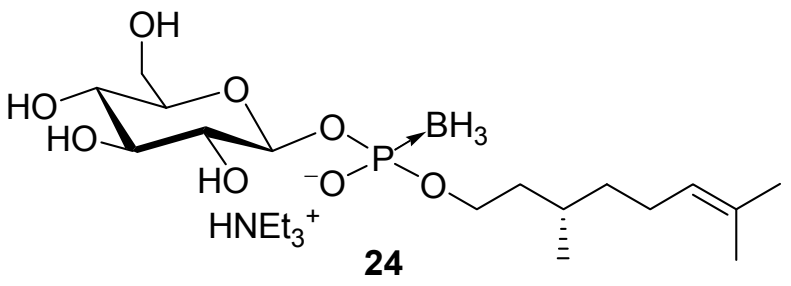

(300 MHz, $\mathrm{CD}_{3} \mathrm{CN}$ )
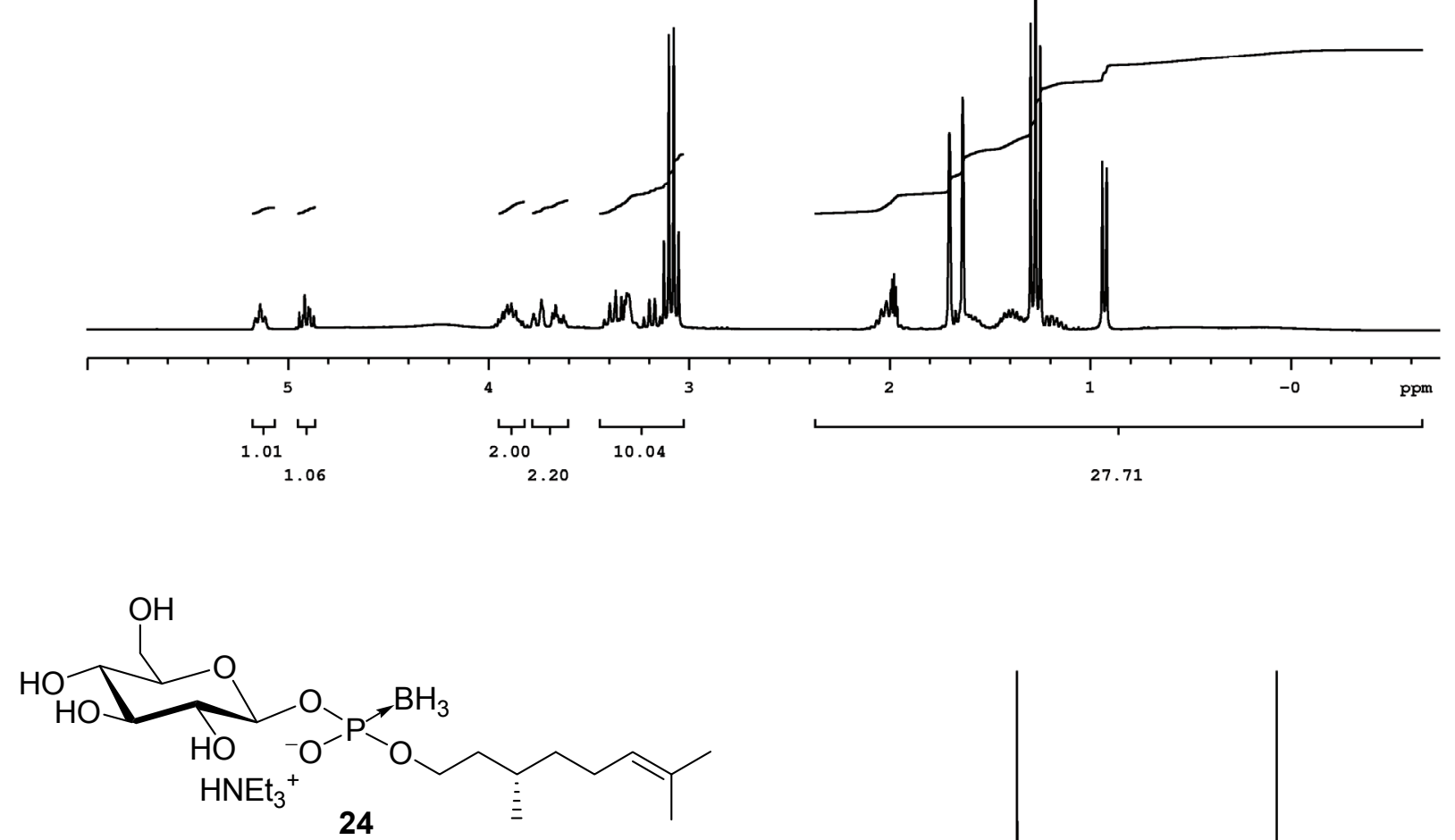

(75.5MHz, $\left.\mathrm{CD}_{3} \mathrm{CN}\right)$

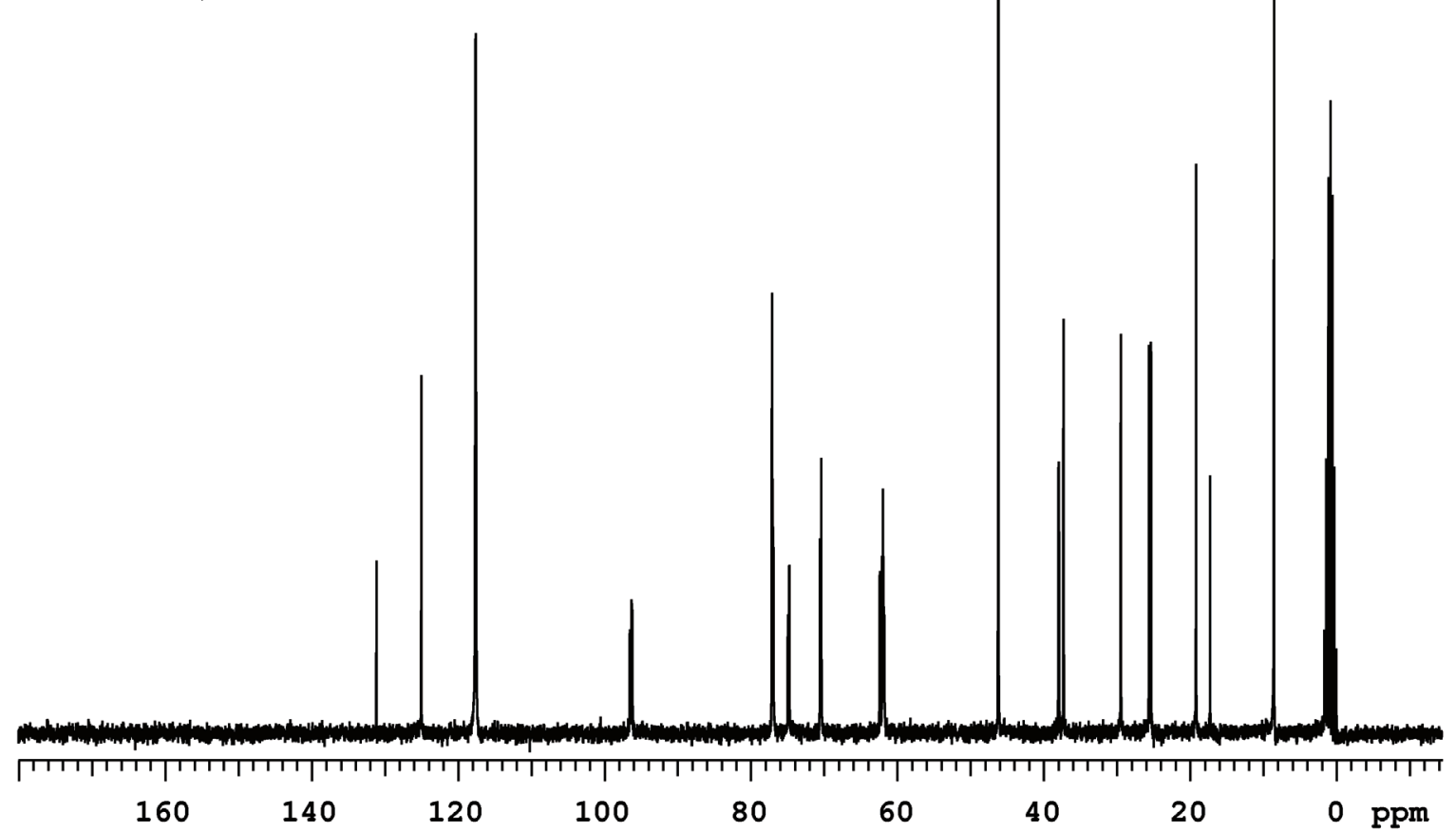




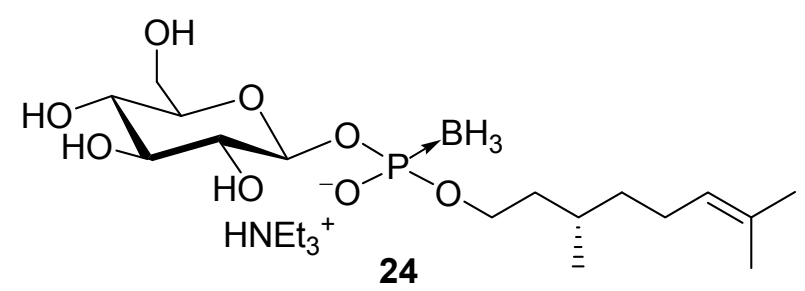

(121.5 MHz, $\mathrm{CD}_{3} \mathrm{CN}$ )
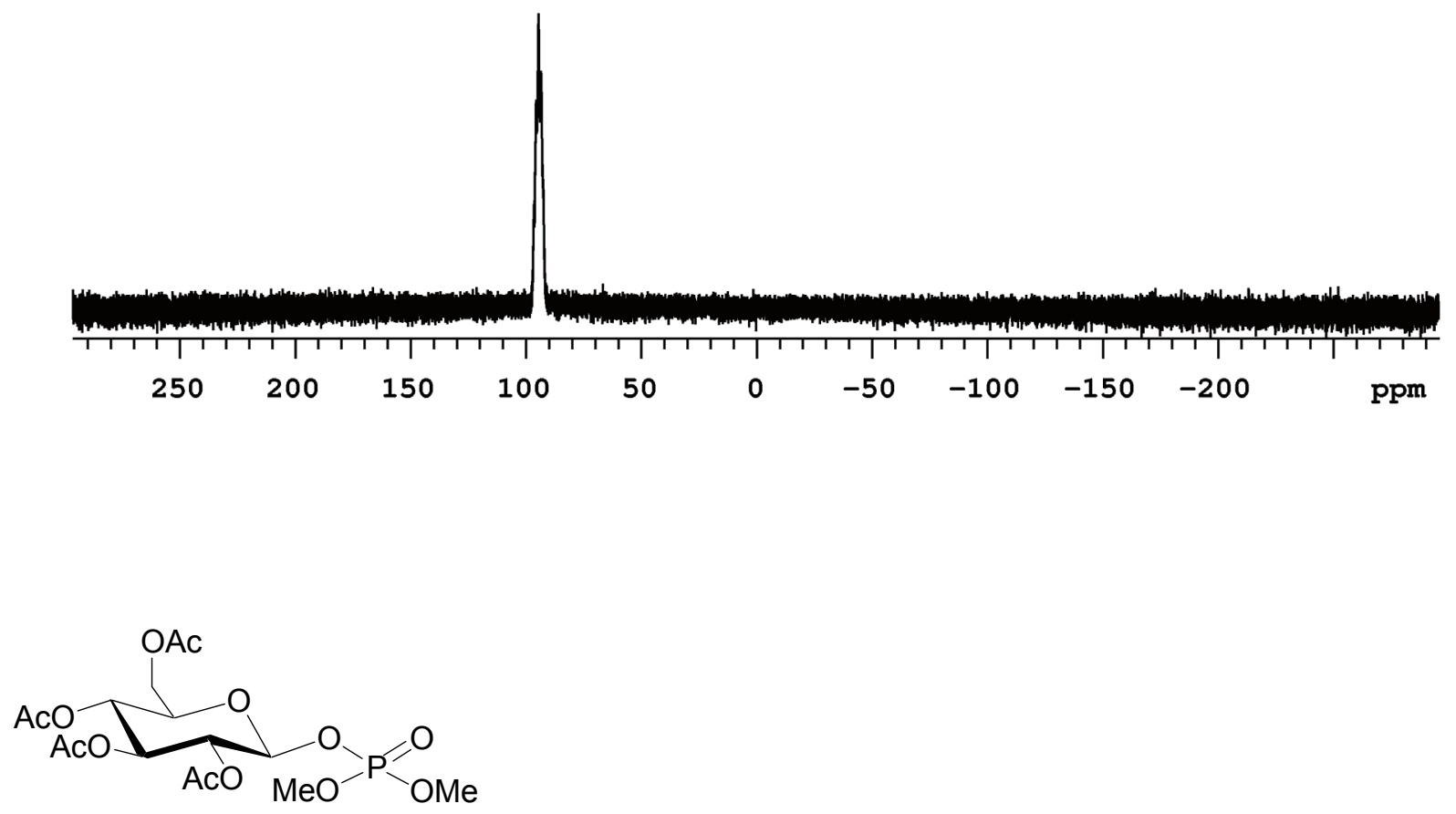

26

(300 MHz, $\mathrm{CDCl}_{3}$ )

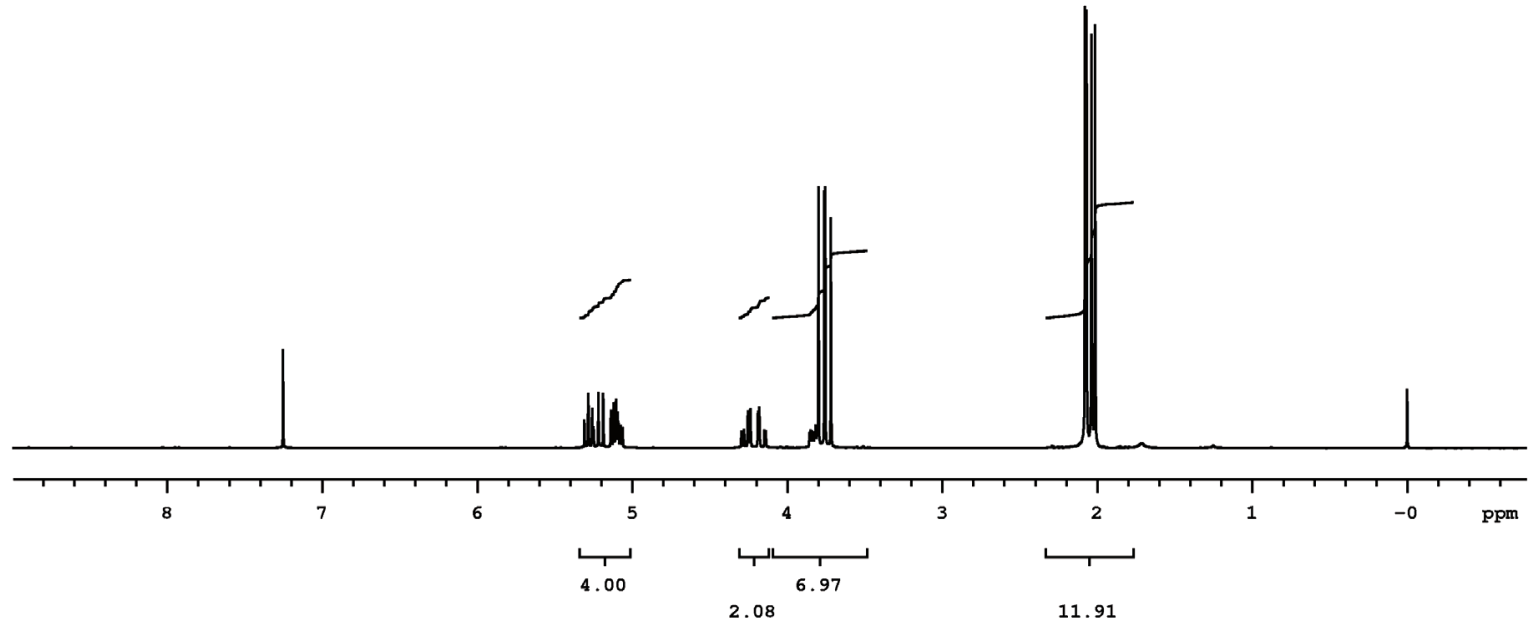



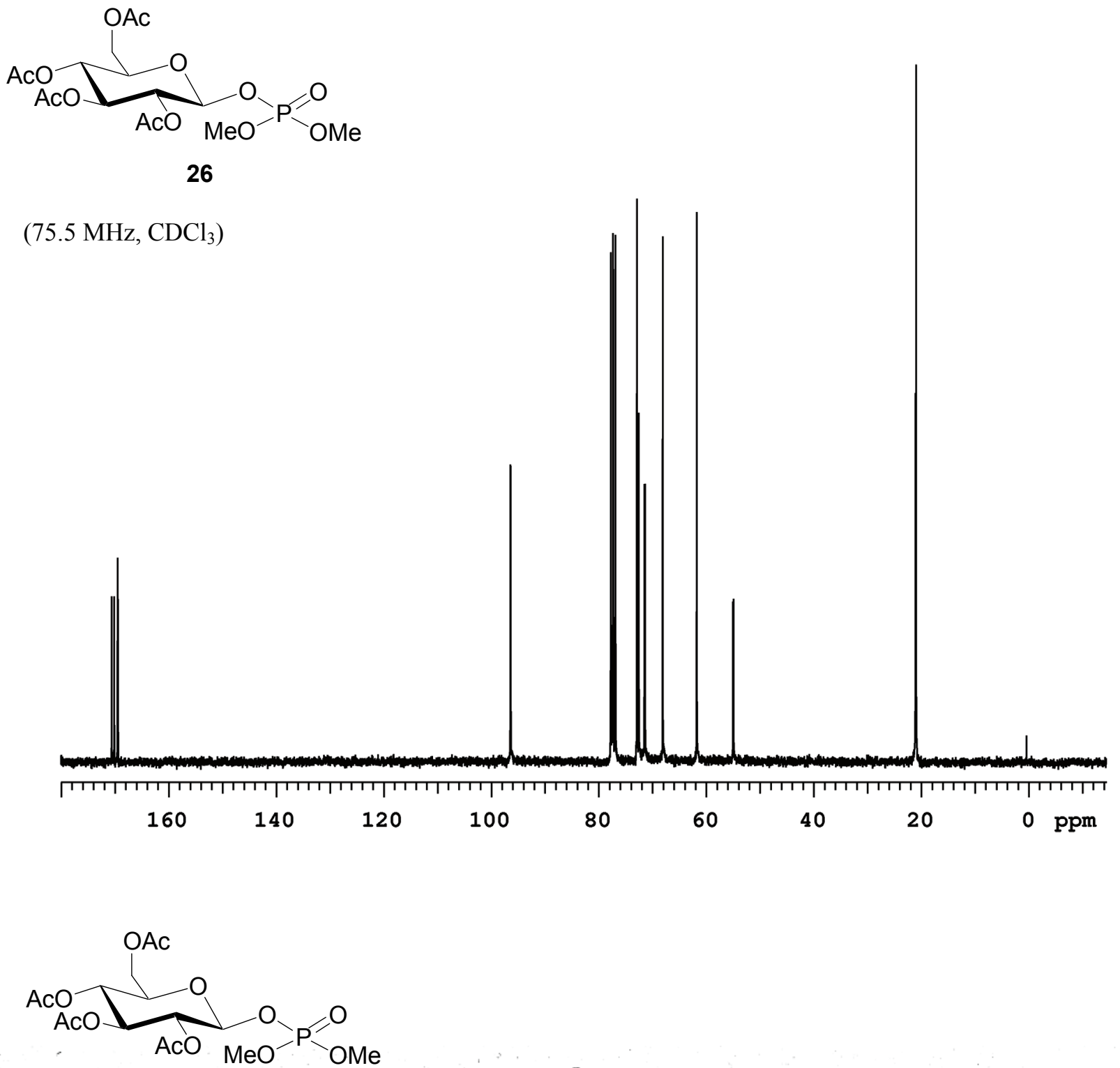

26

(121.5 MHz, $\left.\mathrm{CDCl}_{3}\right)$

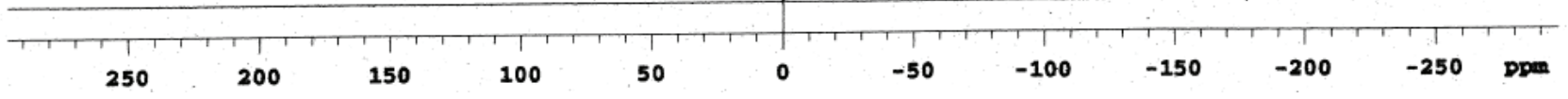

S32 


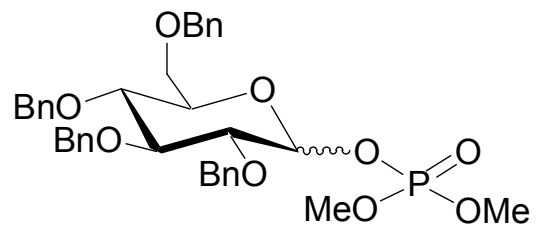

25

(300 MHz, $\mathrm{CDCl}_{3}$ )
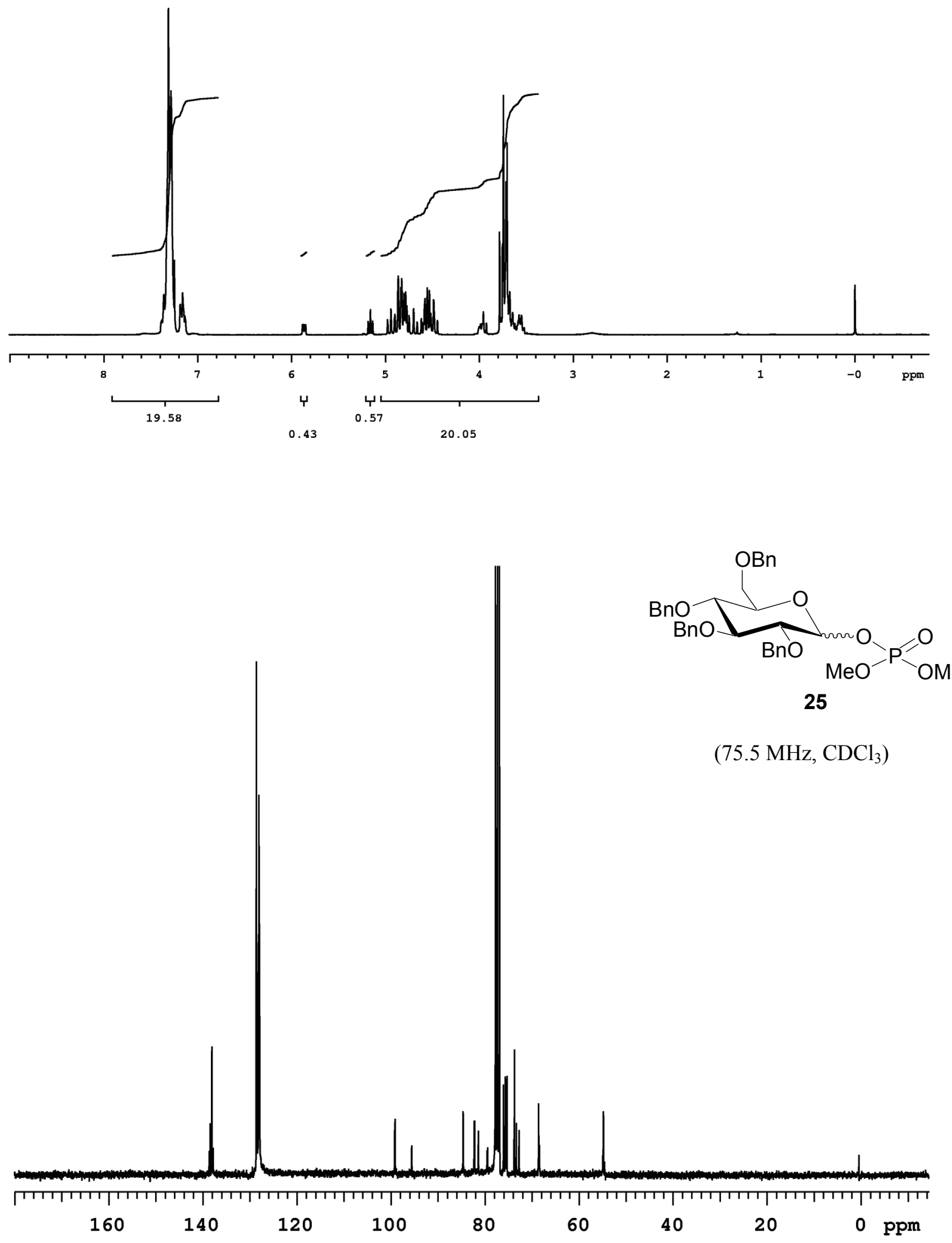


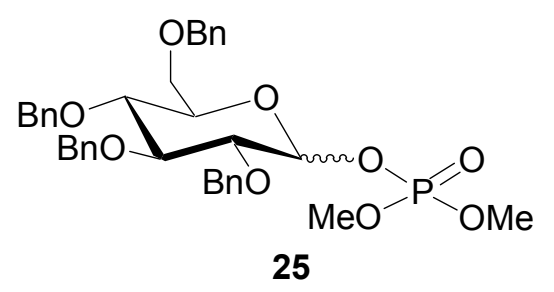

(121.5 MHz, $\left.\mathrm{CDCl}_{3}\right)$
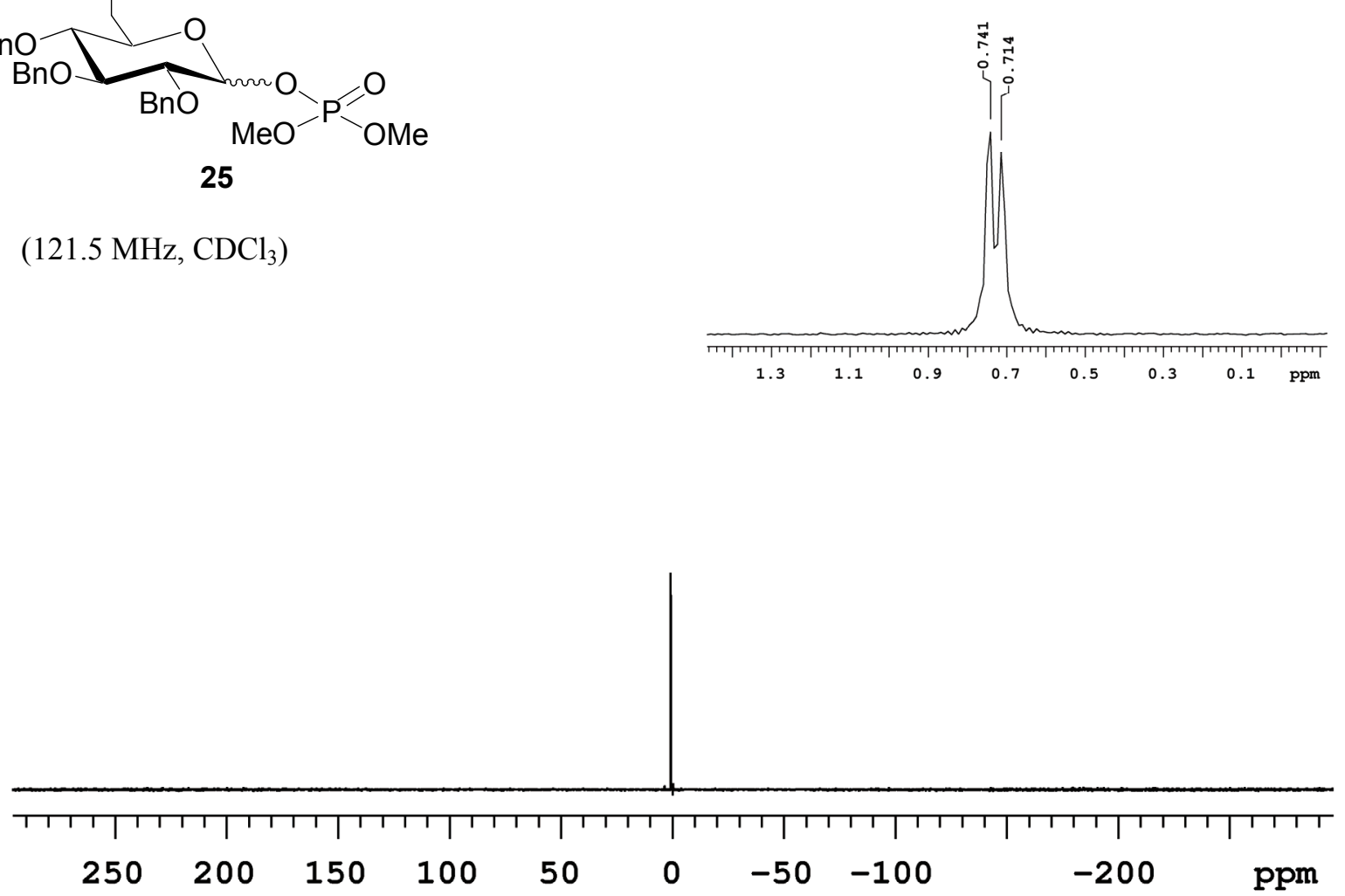